\title{
Improving Clustering Methods By Exploiting Richness Of Text Data
}

by

Abdul Wahid

\author{
A thesis \\ submitted to the Victoria University of Wellington \\ in fulfilment of the \\ requirements for the degree of \\ Doctor of Philosophy \\ in Computer Science. \\ Victoria University of Wellington \\ 2016
}





\begin{abstract}
Clustering is an unsupervised machine learning technique, which involves discovering different clusters (groups) of similar objects in unlabeled data and is generally considered to be a NP hard problem. Clustering methods are widely used in a verity of disciplines for analyzing different types of data, and a small improvement in clustering method can cause a ripple effect in advancing research of multiple fields.

Clustering any type of data is challenging and there are many open research questions. The clustering problem is exacerbated in the case of text data because of the additional challenges such as issues in capturing semantics of a document, handling rich features of text data and dealing with the well known problem of the curse of dimensionality.

In this thesis, we investigate the limitations of existing text clustering methods and address these limitations by providing five new text clustering methods-Query Sense Clustering (QSC), Dirichlet Weighted K-means (DWKM), Multi-View Multi-Objective Evolutionary Algorithm (MMOEA), Multi-objective Document Clustering (MDC) and Multi-Objective MultiView Ensemble Clustering (MOMVEC). These five new clustering methods showed that the use of rich features in text clustering methods could outperform the existing state-of-the-art text clustering methods.

The first new text clustering method QSC exploits user queries (one of the rich features in text data) to generate better quality clusters and cluster labels.

The second text clustering method DWKM uses probability based weighting scheme to formulate a semantically weighted distance measure to improve the clustering results.
\end{abstract}


The third text clustering method MMOEA is based on a multi-objective evolutionary algorithm. MMOEA exploits rich features to generate a diverse set of candidate clustering solutions, and forms a better clustering solution using a cluster-oriented approach.

The fourth and the fifth text clustering method MDC and MOMVEC address the limitations of MMOEA. MDC and MOMVEC differ in terms of the implementation of their multi-objective evolutionary approaches.

All five methods are compared with existing state-of-the-art methods. The results of the comparisons show that the newly developed text clustering methods out-perform existing methods by achieving up to $16 \%$ improvement for some comparisons. In general, almost all newly developed clustering algorithms showed statistically significant improvements over other existing methods.

The key ideas of the thesis highlight that exploiting user queries improves Search Result Clustering(SRC); utilizing rich features in weighting schemes and distance measures improves soft subspace clustering; utilizing multiple views and a multi-objective cluster oriented method improves clustering ensemble methods; and better evolutionary operators and objective functions improve multi-objective evolutionary clustering ensemble methods.

The new text clustering methods introduced in this thesis can be widely applied in various domains that involve analysis of text data. The contributions of this thesis which include five new text clustering methods, will not only help researchers in the data mining field but also to help a wide range of researchers in other fields. 


\section{Acknowledgments}

All praises unto Allah, the most merciful and the beneficent, for all his blessings. I am thankful to all my family members, relatives, friends and colleagues for their kind support, encouragement and love. They where the key people who motivated me to purse a PhD. Also, thanks to my friends who made sure I keep fit and healthy by organizing weekly cricket activities.

I am also thankful for my supervisors Dr. Xiaoying Gao and Dr. Peter Andreae for their guidance throughout my $\mathrm{PhD}$. I will always remain grateful to them for considering me as their student.

Special thanks to Dr. Mengjie Zhang for proof reading some of my work and providing fruitful suggestions. I am honored to be the part of ECRG group which provided me the chance to learn about other student research and an opportunity to make new friends.

Lastly, I would like to thank Victoria University of Wellington for their generous financial support. I am grateful to Victoria University of Wellington for the scholarship and travel grant for the top conferences in different countries. Visiting different conferences provided me an opportunity to present my research work, meet leading scholars of my research area and get the feedback to improve my research work. 


\section{List of Figures}

1.1 Example of multiple possible clusterings . . . . . . . . . . . 2

1.2 Example of overlapping clusters and outliers . . . . . . . 2

1.3 Examples of disjoint views in supspace $\ldots \ldots \ldots 7$

1.4 Generation of multiple clustering . . . . . . . . . . . . . 13

4.1 Comparison of SRC methods based on different evaluation measures . . . . . . . . . . . . . . 57

4.2 S-recall@k on all queries . . . . . . . . . . . . . . . . 58

4.3 S-precision@r on all queries . . . . . . . . . . . . . . . . . . 59

4.4 Processing Time for All Queries . . . . . . . . . . . . . . 61

4.5 RI, ARI and JI Analysis . . . . . . . . . . . . . . . . . . 62

5.1 Traditional subspace vs our clustering approach . . . . . . 66

5.2 A common LDA graphical model using plate notation. . . . 69

5.3 System diagram of DWKM . . . . . . . . . . . . 70

6.1 Process of generating 24 different clustering solutions. . . . . 89

6.2 Sample Clustering . . . . . . . . . . . . . . . . . . 91

6.3 matrix based binary encoding . . . . . . . . . . . . 92

6.4 Precision of the final solution on all datasets . . . . . . . . . . 96

6.5 Recall of the final solution on all datasets . . . . . . . . . . 97

6.6 F1-measure on all datasets . . . . . . . . . . . . . . . . . . 98

6.7 Rand Index on all datasets . . . . . . . . . . . . . . . . 99 
7.1 Example of row-wise crossover method. . . . . . . . . . . 109

7.2 Example of column-wise crossover method. . . . . . . . . . 110

7.3 Example of split and merge mutation method. . . . . . . . 111

7.4 Overview of MOMVEC clustering method . . . . . . . . . . 114

7.5 Analysis of MDC . . . . . . . . . . . . . . . 121

7.6 CA on combined dataset . . . . . . . . . . . . . . 122

7.7 F1 on combined dataset . . . . . . . . . . . . . 123

7.8 RI on combined dataset . . . . . . . . . . . . . . . . . . . 124

7.9 Component wise analysis of MOMVEC . . . . . . . . . . 129

7.10 box plot of Clustering Accuracy on combined dataset . . . . 130

7.11 box plot of F1-measure values on combined dataset . . . . . 131

7.12 box plot of RI values on combined dataset . . . . . . . . . . 132 


\section{List of Tables}

4.1 Weight distribution of the Sense Document Similarity Measure ........................... 50

4.2 The Values of Entropy on a subset of AMBIENT Dataset . . 55

4.3 The Values of Purity on a subset of AMBIENT Dataset . . . . 56

4.4 Cluster labels of STC, LINGO and QSC of the query Jaguar . 60

5.1 Six real world datasets created from 20-Newsgroup dataset . 77

5.2 Comparison of clustering methods on synthetic dataset . . . 78

5.3 Comparison of clustering methods on real dataset . . . . . . 79

5.4 Percentage improvement of DWKM . . . . . . . . . 80

5.5 Percentage improvement of DWKM over FGKM . . . . . . . 81

5.6 P-values of unpaired ttest of DWKM and FGKM . . . . . . 82

6.1 Average ranking of clustering methods of MMOEA . . . . . 101

7.1 Clustering Accuracy computed on 10 two view datasets. . . 118

7.2 F1-measure computed on 10 two view datasets . . . . . . . . 119

7.3 Rand Index computed on 10 two view datasets . . . . . . . . 119

7.4 Improvement of clustering quality of MDC compare . . . . . 120

7.5 Statistical significance test for MDC . . . . . . . . . . 125

7.6 Values of CA computed on 10 two-view datasets . . . . . . . 125

7.7 Values of F1-measure computed on 10 two-view datasets . . 126

7.8 Values of Rand Index computed on 10 two-view datasets . . 126

7.10 Statistical significance test for MOMVEC . . . . . . . . . 128 
7.11 Comparison of MDC and MOMVEC . . . . . . . . . . 133 


\section{Abbreviations}

AHC Agglomerative Hierarchical Clustering. 27

API Application Programming Interface. Glossary: API

BOW Bag Of Words. 22, Glossary: bag of words

DWKM Dirichlet Weighted K-mean. 16

EWKM Entropy Weighted K-means Subspace Clustering. 10

FGKM Feature Group K-means Subspace Clustering. 10

FWKM Feature Weighted K-means Subspace Clustering. 10

LDA Latent Dirichlet Allocation. 16

MDC Multi-objective Document Clustering. 17

MMOEA Dirichlet Weighted K-mean. 16

MMOEA Multi-objective Multi-View Ensemble Clustering. 17

Mock Multi-objective clustering algorithm with K-determination. 85

MOEA Multi-objective Evolutionary Algorithm. 33

NMI Normal Mutual Information. 43

ix 
NSGA-II Non-dominated Sorting Genetic Algorithm. 17, 86

QSC Query Sense Clustering. 15

SL Average Link Agglomerate Hierarchical Clustering Ensemble. 97

SL Complete Link Agglomerate Hierarchical Clustering Ensemble. 97

SL Single Link Agglomerate Hierarchical Clustering Ensemble. 97

SPEA-II Strength Pareto Evolutionary Algorithm. 17

SRC Search Result Clustering. xi, 8

TFIDF Term Frequency Inverse Document Frequency. 48

VSM Vector Space Model. 5, 22, 25, Glossary: VSM 


\section{Contents}

List of Figures $\quad$ v

List of Tables vii

Abbreviations $\quad$ ix

1 Introduction $\quad 1$

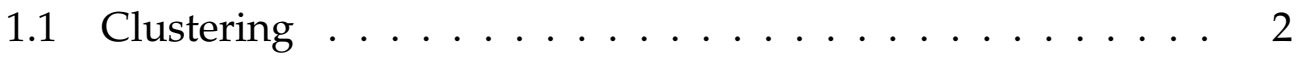

1.2 Clustering Text Data . . . . . . . . . . . . 4

1.2.1 Richness of text data . . . . . . . . . . 4

1.2.2 Multi-View Data ... . . . . . . . . . 5

1.3 Scope ..................... 7

1.3.1 Search Result Clustering . . . . . . . . . . . 8

1.3.2 Subspace Document Clustering . . . . . . . . . . . . . 9

1.3.3 Clustering Ensembles . . . . . . . . . . . . 10

1.4 Research Goals . . . . . . . . . . . . . . . . . . . 13

1.5 Major Contributions . . . . . . . . . . . . . . . 15

1.6 Published Papers and Presentations . . . . . . . . . . 17

1.7 Organization of the thesis . . . . . . . . . 18

2 Background $\quad 21$

2.1 Applications for Text Clustering . . . . . . . . . . . . . . . 21

2.2 Text Representation . . . . . . . . . . . . . . . . . 22

2.3 Similarity Measures . . . . . . . . . . . . . . . 25

xi 
2.4 Clustering Methods . . . . . . . . . . . . . . . . . 25

2.5 Agglomerative Hierarchical Clustering . . . . . . . . . 27

2.6 Centroid Based Clustering Methods . . . . . . . . . . . . 27

2.7 Search Result Clustering . . . . . . . . . . . . . . . 28

2.8 Subspace Clustering . . . . . . . . . . . . . . 30

2.8.1 Hard Subspace Clustering . . . . . . . . . . . . . . . . . . . 30

2.8.2 Soft Subspace Clustering . . . . . . . . . . 30

2.9 Clustering Ensembles . . . . . . . . . . . . . . . . . . 31

2.10 Clustering as an Optimization Problem . . . . . . . . . 32

2.11 Evolutionary algorithms for optimization problems . . . . . 33

2.12 Multi-View Clustering . . . . . . . . . . . . . . . . . 34

2.13 Summary ....................... 35

3 Evaluation of Clustering Algorithms $\quad 37$

3.1 Datasets ........................... 38

3.2 Evaluation Methodology . . . . . . . . . . . . . . . . 40

4 Search Result Clustering $\quad 45$

4.1 Introduction . . . . . . . . . . . . . . . . . 45

4.2 Existing SRC Methods . . . . . . . . . . . . . . . 46

4.3 Representation and Similarity Measure . . . . . . . . . . 48

4.3.1 Document Representation . . . . . . . . . . . . . 48

4.3.2 Document Similarity Measure . . . . . . . . . . . . 49

4.3.3 Query Sense Representation . . . . . . . . . . . . . . . 49

4.3.4 Sense Document Similarity Measure . . . . . . . . 50

4.4 The Algorithm . . . . . . . . . . . . . . . . 50

4.4.1 Step 1: Initial Cluster Generation . . . . . . . . . . 51

4.4.2 Step 2: Cluster Optimization . . . . . . . . . . 52

4.4.3 Step 3: Cluster Ranking . . . . . . . . . . . . 54

4.5 Results ..................... . . 54

4.5 .1 Comparison $1 \ldots \ldots \ldots \ldots 54$

4.5 .2 Comparison $2 \ldots \ldots \ldots 5$ 
4.5.3 Comparison $3 \ldots \ldots \ldots$. . . . . . . . . 56

4.5.4 Further Analysis . . . . . . . . . . . . . . 58

4.6 Summary . . . . . . . . . . . . . . . . . 63

5 Soft Subspace Clustering $\quad 65$

5.1 Introduction . . . . . . . . . . . . . . . . . 65

5.2 Existing Soft Subspace Clustering Algortihms . . . . . . . . . 67

5.2.1 Latent Dirichlet Allocation . . . . . . . . . . . . . 68

5.3 Our LDA Weighted K-Means Model . . . . . . . . . . . . . . 70

5.3 .1 Gibbs Sampling . . . . . . . . . . . . . 70

5.3 .2 Generating $\theta$ and $\phi \ldots \ldots \ldots . \ldots 72$

5.3 .3 Objective Function . . . . . . . . . . . . . . 72

5.3.4 Our Algorithm: DWKM . . . . . . . . . . . . 74

5.4 Experimental Setup . . . . . . . . . . . . . . . 75

5.4 Datasets ................. 76

5.4 .2 Evaluation Measures . . . . . . . . . . 76

5.5 Results ..................... 76

5.5.1 Comparison ................ 78

5.5 .2 Statistical Analysis . . . . . . . . . . . . 82

5.6 Summary . . . . . . . . . . . . . . . . . 83

6 MMOEA $\quad 85$

6.1 Introduction . . . . . . . . . . . . . . . . 85

6.2 Existing Multi-Objective Clustering Methods . . . . . . . . . 87

6.3 Our Multi-View Multi-Objective Evolutionary Approach . . 88

6.3.1 Initialization Method . . . . . . . . . . . . . . . 89

6.3.2 Genetic Representation . . . . . . . . . . . . . 91

6.3 .3 Objective Functions . . . . . . . . . . . . . . . 91

6.3 .4 Crossover ..................... 93

6.3.5 Algorithm: MMOEA . . . . . . . . . . . . . 94

6.4 Evaluation Experiments . . . . . . . . . . . . . . 97

6.5 Results and Discussion . . . . . . . . . . . . . . . . 99 
6.5 .1 Statistical Analysis . . . . . . . . . . . . 100

6.6 Summary . . . . . . . . . . . . . . . . . 102

7 Improving MMOEA 105

7.1 Introduction . . . . . . . . . . . . . . . . 105

7.2 Common Elements of MDC and MOMVEC . . . . . . . . . . 106

7.2.1 Fitness Evaluation . . . . . . . . . . . 107

7.2 .2 Selection . . . . . . . . . . . . . . 109

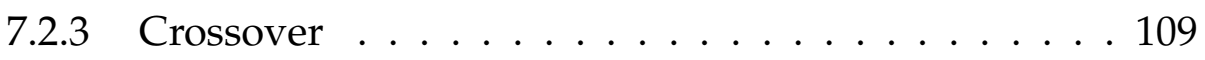

7.2 .4 Mutation . . . . . . . . . . . . . 111

7.2 .5 Tuning ..................... 111

7.3 Multi-objective Document Clustering (MDC) . . . . . . . . 112

7.4 Multi-objective Multi-View Ensemble Clustering (MOMVEC) 112

7.5 Experimental Setup . . . . . . . . . . . . . . . 117

7.5.1 Datasets ................... 117

7.5.2 Evaluation Measure . . . . . . . . . . . . 117

7.6 Experimental Setup for MDC . . . . . . . . . . . . . 117

7.6.1 Comparison on Two View Datasets . . . . . . . . 120

7.6.2 Comparison on Three View Datasets . . . . . . . . . . 122

7.6.3 Statistical Analysis . . . . . . . . . . . . . . 123

7.7 Experimental Setup for MOMVEC . . . . . . . . . . . . . 124

7.7.1 Comparison on Two View Datasets . . . . . . . . . 125

7.7.2 Comparison on Three View Datasets . . . . . . . . . . 130

7.7.3 Statistical Analysis . . . . . . . . . . . . 131

7.8 Single vs Multi-Objective Analysis . . . . . . . . . . . . . . 132

7.9 Summary . . . . . . . . . . . . . . . . . . 133

8 Conclusions 135

8.1 Objectives and the Contributions . . . . . . . . . . . . 135

8.2 Main Conclusions . . . . . . . . . . . . . . . . . . . 138

8.3 Limitations and Future Work . . . . . . . . . . . . . 141

8.3.1 Future Work on Finding Multiple Views . . . . . . . . 143 
CONTENTS

8.4 Closing Remarks . . . . . . . . . . . . . . . 143

$\begin{array}{ll}\text { Appendices } & 145\end{array}$

$\begin{array}{ll}\text { Glossary } & 147\end{array}$

$\begin{array}{ll}\text { References } & 148\end{array}$ 


\section{Chapter 1}

\section{Introduction}

Organizing similar objects into different groups provides an opportunity to learn about the structure of the data. Generally, objects are grouped together by considering the common characteristics of the objects. For example documents that share common words can be clustered together and the records of patients that provide similar symptoms can be clustered together. The former example, can be useful to create a library catalog whereas the later example can be useful to prescribe similar treatments for patients.

In computer science, clustering or cluster analysis is an unsupervised machine learning technique inspired from the human ability to group similar objects in unlabeled data. Unlabeled data generally refers to raw data or data without the information of the groups or classes. Clustering analyzes the data and discovers hidden structure of the data by grouping similar data objects based on some notion of similarity. The main aim of clustering is to have better understanding of the data by learning from the structure of data obtained from clustering.

Clustering has received enormous attention in the research community because of its challenging problems and a wide range of applications $[52,61]$. It has been investigated for decades and research is still going on in a wide range of disciplines such as data mining, machine learning, 


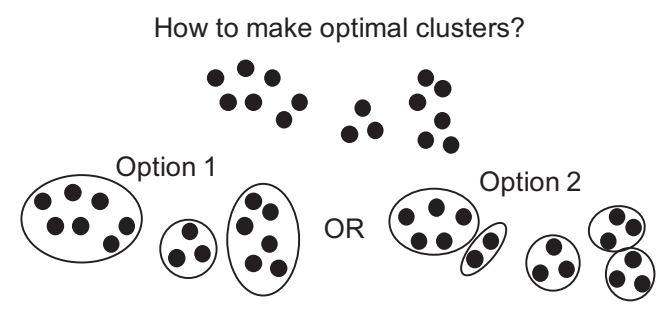

Figure 1.1: Example of multiple possible clusterings

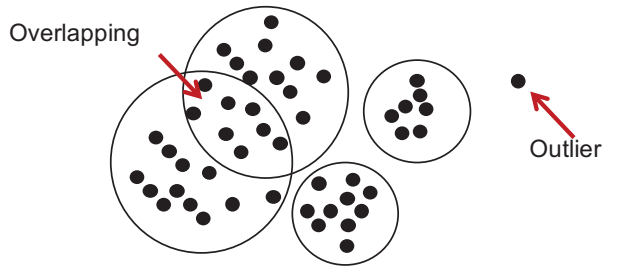

Figure 1.2: Example of overlapping clusters and outliers

pattern recognition, image analysis, information retrieval, bioinformatics, theoretical computer science and statistics. General applications that can benefit from such clustering include document analysis, gene expression analysis, sensor surveillance, customer segmentation and image segmentation [52]. Since clustering methods are widely applied in various fields of science to perform data analysis, a small step towards improving clustering methods can be a catalyst for advancement in different fields and can initiate new directions for the researchers.

\subsection{Clustering}

Grouping similar objects into different clusters is generally referred as the clustering problem or the clustering task. In 1957, Stuart Lloyd developed a clustering method named $k$-means [91] for automatically finding groups in data and to address the clustering problem. Since then thousands of variations of $\mathrm{k}$-means and new clustering methods have been proposed. However, due to the ill-posed nature of the clustering problem, clustering still has many challenges [75]. For example, Figure 1.1 depicts the problem of multiple possible clustering solutions with different levels (i.e. three big clusters or five small clusters) of the same data. Figure 1.2 depicts the problem of overlapping objects in clusters and objects that do not belong to any clusters (generally referred to as outliers). 
In Figure 1.1, there are two possible clustering solutions (option 1 and option 2) to the clustering problem. Deciding which option is better than the other does not have a straightforward solution. In some cases, having big clusters is preferred and in other cases, having small clusters are preferred. One way to address this issue is to check the compactness (similarity of objects within clusters) of the clusters in a clustering solution. Determining the compactness of the clusters depends upon the way of measuring the distance between objects. A poor choice of a distance measure may lead to grouping of objects that are not very similar to each other, and results in a bad quality clustering solution. Furthermore, even with a good choice of a distance measure, in some cases different clustering solutions might have the same compactness. Hence, the problem of multiple clustering solutions still lingers. Also the two clustering solutions in Figure 1.1 might provide two different insights of the data and it may be better to produce two clustering solutions instead of one.

Since real world objects can belong to more than one cluster, it is often desired for a clustering method to produce overlapping clusters. The overlapping cluster depicted in Figure 1.2 often requires a threshold parameter. The value of this threshold depends on the question how much overlapping should be allowed.

Traditional algorithms such as k-means restrict objects to only one cluster to avoid setting the value of a threshold. Other clustering methods generally choose the value of a threshold based on intuition or after conducting various experiments on the datasets. This might not be a good approach as different datasets will need different threshold values.

Figure 1.2 depicts an object, which is not assigned or covered by any cluster, referred as an outlier. Outliers are very common in noisy data ${ }^{1}$ and can result in poor compactness of the overall clustering solution. Hence, a clustering method also has to address the outliers in such a way that the compactness of a clustering solution is not compromised.

\footnotetext{
${ }^{1}$ noisy data refers to data points that do not follow the underlying pattern
} 


\subsection{Clustering Text Data}

Traditional clustering methods generally address data described in terms of numeric or nominal attributes $[58,74,79,103,150,91]$. Traditional clustering methods can be extended to cluster text data such as documents. Text can be represented as a binary data, where a word in a document (or text segment) is treated as a binary feature based on the presence/absence of the word in the document. Clustering methods for nominal data can use this binary representation to cluster the documents [79]. A better text representation which involves weighting schemes based on the frequencies of the words in a document and frequencies of words in the collection of all the documents can also be used in traditional clustering methods to group similar documents.

However, a recent survey of text clustering methods argues that general purpose clustering methods such as k-means should not be used to cluster text data [4]. The survey is mainly focused on describing existing text clustering methods. However, it also highlights that text has rich features with unique characteristics which need to be considered in clustering text data, and raises unanswered questions such as what are the rich features in text?, how do we represent rich features? and how can we exploit rich features for improving clustering methods?

Upon further study, we observed that the existing literature only touches the tip of an iceberg when it comes to exploiting rich features for clustering text data.

\subsubsection{Richness of text data}

The richness of text data involves syntactic, semantic and structural features of the text [44]. An example of syntactic features are word-frequencies (number of time a word occurred) in the documents. Examples of semantic features are synonyms (words having same meaning), phrases, ambiguous words/phrases (words/phrases with multiple meanings), top- 
ics (meta features), contextual information of the text etc. An example of structural features are markups of the text such as title, headings, hypertext (weblinks) etc. In addition, text data can have domain specific features, for example, a user query in a result set return by search engines, hashtags in twitter text data, citations in research articles etc.

Since clustering text involves rich data [44], it exacerbates the clustering problem by adding the extra challenges of dealing with rich features. One of the key challenges in dealing with text data is to transform or represent the rich data. Another major challenge is to measure the similarity between two documents.

Representing text data using Vector Space Model (VSM) assumes words are independent, unordered and have no context (i.e. it ignores the semantic information) [11]. However, these assumptions are not realistic and often mislead and result in poor quality clustering solutions.

The different representations of the text data can result in different clustering solutions e.g. clustering research articles by only considering the citations of the articles will produce one possible set of clusters and clustering research articles by only considering the contents of the article could result in another possible sets of clusters. Also, both clustering solutions could provide different insights about the research articles. Unfortunately in different clustering methods, the different representation of the text data or the rich features (which can be used to represent data) are not generally utilized, therefore, a poor quality clustering solutions is often produced by the clustering methods.

\subsubsection{Multi-View Data}

Documents in text data are generally represented using only syntactic features. Recent approaches have used semantic and structural features along with syntactics features for document representations. However, the use of multiple features at the same time in text clustering is rare. The multiple 
representations of data are also known as multi-view data [143, 121].

Multi-view data or multiple views refer to different representations or perspectives of the same data instance. Generally each view may have distinct statistical properties or formulations that lead to different insights of the data. Following are the examples of views in different domains.

- In analysis of scientific documents, the views are syntactical features such as words of documents, network graph of references/citations, description of the documents such as title, author, conferences and all other possible ways to represent a document.

- In customer segmentation, the views are the different set of customer behavior such as attributes related to sports activities, food consumption, and music genre etc.

- In a gene expression analysis, the views are different functional roles of genes.

- In sensor surveillance, the views are different measurements of sensor nodes.

- In image segmentation, the views are different subsets of pixel features.

In general, objects that can be represented in multiple ways by different features can provide different perspectives or views of the data. Furthermore, views can be based on homogeneous data with different subsets of features or heterogeneous data of different types of features. Heterogeneous data of different features in web pages include: words in documents; publishing date of a web page; average visitor time on the web page; and revenue generated per month from displaying ads on the web page. Another example of heterogeneous data of different features is scientific publication analysis, where documents can be represented in a vector space (word-frequency) as well as graph space (based on the citations). 

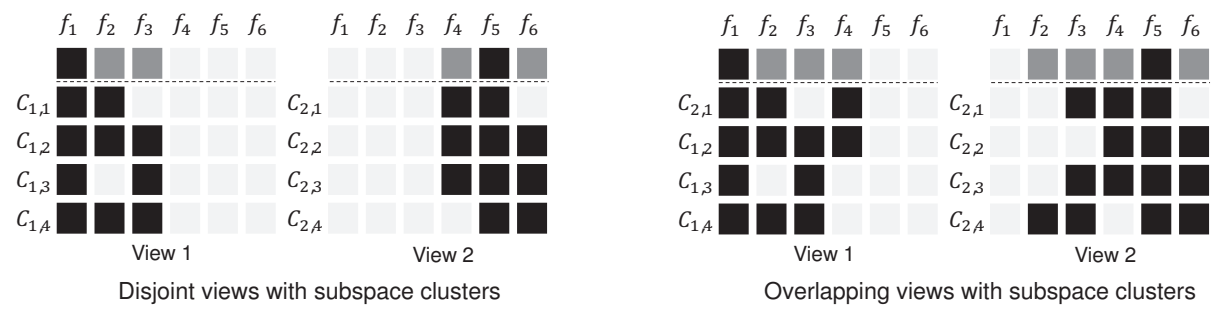

Figure 1.3: Examples of disjoint and overlapping views in subspace (a subset of features).

In addition, a subset of features or subspaces can also provide a distinct perspective of the data. Hence, the views can also be based on subsets of features.

Figure 1.3 shows different types of views that can be found in the subset of features. The black squares on the first row show the specific feature is present in all clusters. Whereas, the light gray squares on the first row show the specific feature is present in particular clusters. A black square next to the clusters (rows 2-5) show that a feature $f$ is present in the cluster $C$. The disjoint views consist of different subset of features $\left(f_{1}, f_{2}, f_{3}\right.$ represent one view and $f_{4}, f_{5}, f_{6}$ represent another view) and do not share any features. Overlapping views, share one or more features that are already included in the different views.

As said earlier, different views may lead to a different set of clusters and can provide different insights of the data. Generally previous text clustering approaches only considered one view of the data for clustering. This thesis will focus on using multiple views to improve clustering methods.

\subsection{Scope}

Addressing all the challenges of clustering data is too broad for this thesis. Therefore, the scope of this thesis is restricted to the following clustering 
approaches for text data.

\subsubsection{Search Result Clustering}

The rich features vary from domain to domain in the development of special purpose text clustering methods. For example, user query in Search Result Clustering (SRC) is a rich feature which is not present in general clustering of documents. SRC is a special purpose clustering approach which groups the results of a user query in such a way that each cluster represents a set of related results. To be useful to the user, the different cluster should contain the results corresponding to different possible meanings of the user query and the cluster labels should reflect these meanings. However, existing SRC methods often ignore the utilization of the user query and group the results based on the similarity of search results.

\section{Limitations of Search Result Clustering}

Existing SRC methods are vulnerable to the two problems: low quality cluster, where the results within a single cluster are related to different meanings of the query; and poor cluster labels, where the label of the cluster does not reflect the query meaning associated with the results in the cluster.

Furthermore, the similarity between two documents is often based on word frequency i.e. only syntactic features of the documents are considered. Such similarity measures are regarded as syntactic measures because they only consider counts of words. Since documents contain both syntactic and semantic features, the under utilization of rich features (semantic features) can degrade the overall cluster quality (low compactness of clusters).

SRC clustering methods can be improved by addressing the problems of low cluster quality, poor cluster labels and under utilization of rich features. Hence, a new SRC method is desired which can address the current problems of existing SRC methods. 


\subsubsection{Subspace Document Clustering}

Subspace document clustering approach has been widely applied on general purpose document clusterings. The documents in general purpose clustering are assumed to have medium length, the clusters are unbalanced, the data is noisy and unlike Search result clustering, there is no user query. The documents can be the collection of news articles, web pages, emails, research articles etc.

Traditional clustering methods such as k-means, consider all features at the same time to cluster the data and are only suitable for data with a small number of features. In text datasets, the number of features is very large and it is more suitable to find clusters in subsets of features (subspaces) instead of considering all the features at once (full space). The most popular clustering approach which tries to find clusters in subspaces is called subspace clustering.

Subspace clustering is often divided into two sub-steps. The step one determines the subspaces and step two clusters the data. Based on how these steps are performed, there are two main categories of subspace clustering methods: hard subspace clustering and soft subspace clustering [26, 135]. In hard subspace clustering, a feature in a subspace is either present or not present ( 1 or 0$)$, whereas in soft subspace clustering, a feature in a subspace is determined by its degree of presence (i.e. a weight between $0-1)$. A feature is considered relevant if its weight is high in a subspace and considered irrelevant if its weight is low in a subspace.

In text datasets, some features can be considered to be partially presented in subspaces. Therefore, soft subspace clustering methods are becoming more popular in text clustering.

The most popular soft subspace methods are Feature Weighted K-means Subspace Clustering (FWKM) [78], Entropy Weighted K-means Subspace Subspace Clustering (FGKM) [77] and Feature Group K-means Subspace Clustering (FGKM) [26]. These methods use modified versions of $k$-means algorithm for clustering the data in different subspaces based on their fea- 
ture weights. These methods mainly differ in terms of how they compute the feature weights.

\section{Limitations of Subspace Document Clustering}

Common subspace clustering methods are heavily dependent upon the distance measure for grouping the objects. The previous approaches in applying subspace clustering in documents only use the syntactic information and ignore the rich features of the text (semantic information of the documents). Therefore, we believe that clustering documents using subspace clustering approach requires a special distance measure which not only considers the syntactic information of the documents but also takes the semantic information into account.

\subsubsection{Clustering Ensembles}

Thousands of clustering methods have been proposed in the last 50 years [75]. Each clustering method is unique in its own way and generally produces different sets of clusters. To leverage the advantages of different clustering methods, researchers have been trying different techniques for combining different clustering methods. This type of clustering approach is commonly known as clustering ensembles. Clustering ensembles is an emerging clustering approach which aims at deriving a superior solution (having better compactness) by considering the multiple clustering solutions that are generated from different clustering methods.

\section{Limitations of Clustering Ensembles}

Clustering ensembles methods for text clustering include two important steps: 1) generating a set of candidate clustering solutions and 2) combining the set of candidate clustering solutions to generate a final clustering solution. Therefore, the key issues that clustering ensemble approaches 
address are how to generate different candidate solutions and how to combine them.

A common practice for step 1 is to use different clustering methods (kmeans, Agglomerate Hierarchical Clustering, etc) with different parameters to generate multiple clustering solutions. This is a good strategy if the data can only be represented from one view. However, in the case of textual data, a document can be represented by multiple views e.g., semantic view (i.e. topics, title, hypertext etc.) and a syntactic view (i.e. term frequencies). Considering multiple views at the same time has already proven to result in better cluster quality [13]. Therefore, it seems reasonable that using multiple views for generating different candidate in a clustering ensembles method will result in generating a diverse set of candidate clustering solutions.

The use of multiple views in clustering raises a third issue of how to detect multiple views in the data. Generally, the views are pre-defined by the domain experts. However, in the absence of domain experts the views needs to be automatically generated. The detailed description of the three issues of clustering ensembles how to generate different candidate solutions, how to combine them, and how to detect multiple views are as follows.

- How to generate different candidate solutions? Different clustering methods or the same method with different parameters can be used along with different representations, a subset of objects, and projections on subspaces of the objects to generate different clustering solutions. Figure 1.4 shows different ways for generating the clustering solutions. However, these methods may produce redundant clusters. Also, candidate clustering solutions are generally mixture of high quality and low quality clusters. In order to achieve high quality clusters and avoid redundant clusters, the candidate clustering solutions need to provide additional knowledge for performing cluster analysis from different perspectives.

Multiple views provide different ways to represent the data and based 
on these views different candidate clustering solutions could be generated. The different candidate clustering solutions provides different insights about the data and could be useful to analyze the data from different perspectives. Therefore, it seems reasonable to develop a new clustering method which can use multiple views of the data to generate a diverse set of candidate clustering solutions.

- how to combine different candidate solutions? In clustering ensembles, these different candidate clustering solutions are merged to one single solution by deriving a consensus between the clustering solutions. There are different approaches for developing a consensus function for clustering ensembles. The most prominent and successful approach for consensus function for the clustering ensembles is called median partition.

The median partition approach tries to solve an optimization problem of the median partition with respect to the clustering. In clustering, the median partition is defined as a clustering solution that is most similar to all clustering solutions.

Generally all clustering ensemble based consensus approaches, ignore a key point that a set of candidate clustering solutions are composed of high quality ${ }^{2}$ and low quality clusters. Moreover, the current approaches try to select a single candidate clustering solution, instead of generating a final clustering solutions by considering only high quality clusters from different candidate clustering solutions.

In order to generate a better clustering solution, a new consensus function is desired, which considers only high quality clusters from different candidate clustering solutions.

- how to detect multiple views? Given that multiple views are good for generating candidate clustering solutions, one interlinked chal-

\footnotetext{
${ }^{2}$ high quality clusters are the ones that have high intra-cluster similarity and are dissimilar from other clusters
} 


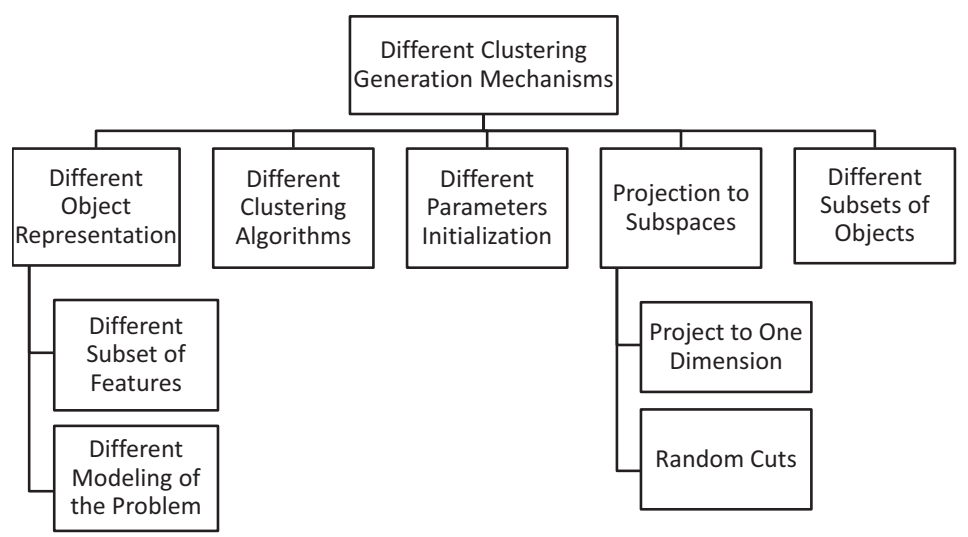

Figure 1.4: Generation of multiple clustering

lenge in utilizing the rich features in clustering methods is to determine the multiple representations of views of the data. Detecting views which provide the different representations of the data is quite hard and there is no known method that can accurately address this problem. The best way to detect multiple views is to use domain experts. Therefore, we restrict the scope of this thesis to use predefined views by domain experts.

\subsection{Research Goals}

The overall goal of this thesis is to develop new and improved clustering methods for text data and demonstrate that exploiting the richness of text data can improve the performance of clustering methods. In order to achieve this goal, not only we must address the general challenges involved in developing clustering methods but also we must address the challenges in handling text data. We set the following research objectives to guide the research work in this thesis that addresses the limitations of existing clustering approaches:

1. Develop a new clustering method to exploit user queries for Search Result Clustering(SRC). 
The thesis addresses this objective by developing a new SRC method that exploits the user query and uses both syntactic and semantic features of the search results to construct clusters and labels. The newly developed SRC method is compared with existing state-ofthe-art SRC methods to show that our SRC method which uses different query senses generates a good candidates (more semantically meaningful labels) for the cluster labels and can also lead to high quality clusters.

2. Develop a new subspace clustering method for text data that uses semantic information in its distance measure.

The thesis addresses this objective by developing a new soft subspace clustering method based on semantic feature weighting and developing a semantically weighted distance measure. The thesis also discusses different weighting schemes for soft subspace clustering methods and present results of comparing our newly developed soft subspace clustering method against existing soft subspace clustering methods.

3. Use multiple views of text data to generate diverse candidate clustering solutions for clustering ensemble methods.

The thesis addresses this objective by developing a new clustering ensemble method that exploits multiple views to generate different clustering solutions. The thesis also describes the results of comparison between our newly developed clustering ensemble with existing methods to proof the assumption that multiple views can influence the overall quality of the clustering solution.

4. Develop a new approach for combining different candidate clustering solutions to generate the final clustering solution for clustering ensemble methods.

The thesis addresses this objective by developing a new method for clustering ensembles based on evolutionary approach which derives 
a final clustering solution by selecting a combination of high quality clusters from candidate clustering solutions instead of selecting the single candidate clustering solution. The thesis also presents results of comparing our newly developed method with existing clustering approaches.

\subsection{Major Contributions}

This thesis contributed five newly developed text clustering methods which not only address some of the general issues in text clustering but also use the richness of the text to produce improved clustering solutions. The thesis points to new prospects in clustering the text data by considering the richness of features and exploiting multiple views of the text data. The following are the main contributions of the thesis.

1. The first contribution is a new description-centric search result clustering method Query Sense Clustering (QSC) which exploits query senses to generate meaningful cluster labels and uses syntactic and semantic features of documents to generate quality clusters [131]. QSC outperforms existing state-of-the-art Search Result clustering methods. QSC is computationally inexpensive and provides better quality clusters with meaningful labels as compared to other algorithms, hence it has the potential to be applied to real time search result clustering applications.

2. The second contribution is a new soft subspace clustering method Dirichlet Weighted K-mean (DWKM), which uses the Latent Dirichlet Allocation (LDA) model to weight the features in the subspaces for clustering the documents [135]. The LDA model was implemented using a standard Gibbs sampling algorithm. Two matrices, topicterm and topic-documents, were generated. We used the topic-term matrix to develop a new weighted distance measure, where topics 
are used as subspaces. The algorithm is initialized using the topicdocument matrix, where topics are considered as initial clusters. The main innovation of DWKM is the use of semantic information in feature weighting and a novel semantically weighted distance measure. Experiments show that DWKM has made a include statistically significant improvement over recently developed soft subspace clustering methods on both synthetic and real-world datasets.

3. The third contribution is a multi-objective approach for clustering ensembles , Multi-view Multi-objective Evolutionary Algorithm (MMOEA), which uses multiple views to generate a set of candidate solutions and selects high-quality overlapping clusters from the candidate solutions to form a superior clustering solution [132]. MMOEA uses multiple views to generate an initial set of candidate clustering solutions. This results in diverse candidate clustering solutions having mixtures of very high quality clusters and low quality clusters. MMOEA provides a novel method for generating a final clustering solution by selecting and combining the individual clusters from different candidate clustering solutions. Another major novelty of MMOEA is to use the multi-objective ranking system from NSGAII to guide the optimization to address the conflict between the objective criteria used in MMOEA algorithm. The experiments have shown that MMOEA outperformed other existing cluster ensemble methods.

4. The fourth contribution is two new clustering ensemble methods Multi-objective Document Clustering (MDC) and Multi-objective Multi-View Ensemble Clustering (MOMVEC) based on Strength Pareto Evolutionary Algorithm (SPEA-II) and Non-dominated Sorting Genetic Algorithm (NSGA-II) respectively $[133,134]$. The new methods have four new innovations: weighted intra-cluster distance in objective functions, row/column wise crossover methods, split/merge 
mutation methods and a tuning method in the multi-objective evolutionary process for refining the clustering solution.

Both MDC and MOMVEC are able to produce both overlapping and non-overlapping clusters and are able to automatically determine the number of clusters for final clustering solution.

MOMVEC outperformed all other clustering ensemble methods including MMOEA and showed a slightly better performance than MDC.

\subsection{Published Papers and Presentations}

Parts of this thesis were published and presented in the conferences during the course of research. The list is as follows:

1. Wahid, Abdul, Xiaoying Gao, and Peter Andreae. "Multi-Objective Clustering Ensemble for High-Dimensional Data based on Strength Pareto Evolutionary Algorithm (SPEA-II)." Data Science and Advanced Analytics (DSAA), 2015. 36678 2015. IEEE International Conference on. IEEE, 2015.

2. Wahid, Abdul, Xiaoying Gao, and Peter Andreae. "A Soft Subspace Clustering Method for Text Data using Latent Dirichlet Allocation based Feature Weighting Scheme." Web Information Systems EngineeringWISE 2015. Springer International Publishing, 2015. 124-138.

3. Wahid, Abdul, Xiaoying Gao, and Peter Andreae. "Multi-Objective Multi-View Clustering Ensemble based on Evolutionary Approach." Evolutionary Computation (CEC), 2015 IEEE Congress on. IEEE, 2015.

4. Wahid, Abdul, Xiaoying Gao, and Peter Andreae. "Multi-view clustering of web documents using the multi-objective genetic algorithm." Evolutionary Computation (CEC), 2014 IEEE Congress on. IEEE, 2014. 
5. Wahid, Abdul, Xiaoying Gao, and Peter Andreae. "Exploiting User Queries for Search Result Clustering." Web Information Systems EngineeringWISE 2013. Springer Berlin Heidelberg, 2013. 111-120.

6. Wahid, Abdul, Xiaoying Gao, and Peter Andreae. "Context Aware Web Page Clustering." New Zealand Computer Science Research Student Conference-NZCSRSC 2013.

\subsection{Organization of the thesis}

The thesis is organized into multiple chapters and a brief description of the chapters are as follows:

Chapter 2 discusses the general terminologies, the background of general clustering techniques for text data. It will also include the basic introduction of Subspace clustering, Multi-view clustering and Ensemble clustering.

Chapter 3 describes an overall methodology for evaluating the newly developed clustering methods. It includes the description of text datasets and evaluation measures for all experiments. Specific experimental parameters for the experiments are discussed in their relevant chapters.

Chapter 4 describes the first problem in more detail with relevant literature review. It describes a new clustering method named Query Sense Clustering (QSC) and a comparative study of its results with other clustering methods. It also includes a discussion section which describes the contribution and achievements of the first goal of the thesis.

Chapter 5 focuses on the problem of high-dimensionality in text data and semantic distance measures. It includes the relevant literature review, a new clustering method named Dirichlet Weighted k-means (DWKM) and a comparative study of its results with other clustering methods. The contribution and achievements of the second goal of the thesis are also provided at the end of the chapter. 
Chapter 6 discusses the multiple views in text data and presents a new clustering ensemble method named MMOEA. It outlines the relevant related work and discuss the implementation of MMOEA. The comparison of MMOEA and other existing clustering methods are also included in this chapter along with a discussion of the achievements of third and fourth objectives.

Chapter 7 provides two improved version of the ensemble clustering method MMOEA called MDC and MOMVEC. The implementation of MDC highlights the importance of better genetic operators and the fitness function used in the evolutionary process. The chapter describes the implementation of MDC and its performance comparison with other existing methods including MOMVEC and MMOEA. The last section of the chapter discusses on the contribution of the research work, and its relevance to third and fourth objectives of the thesis.

Chapter 8 concludes the thesis by summarizing all the presented clustering methods. Also, the chapter discusses major findings of the experiments and highlight the important contributions. The last section of the chapter points out limitation, further research directions for this thesis and new pointers for text clustering. 


\section{Chapter 2}

\section{Background}

This chapter presents the basic concepts and methods of text clustering. In particular, this chapter describes different ways of representing text data, different similarity measures to compare documents and an overview of general text clustering methods.

\subsection{Applications for Text Clustering}

Generally, text clustering is applied as a first step in different real world applications to learn the structure of the data. However, text clustering is also used for dimensionality reduction and topic modeling. The most prominent applications for text clustering include search engines, document analysis, topic modeling and document summarization.

In search engines, text clustering plays a vital role in improving the performance of document retrieval by indexing or grouping similar documents. A typical search engine takes a user query as input, searches the complete corpus and shows the matched or relevant documents as a result. However, when text clustering method is applied for indexing, the search engine only searches the small chunk or the small corpus which is relevant to the user query. Hence, the response time of the search engine is heavily reduced. Almost all modern search engines apply some sort of 
clustering algorithms for fast information retrieval.

In document analysis, text clustering is generally applied to learn the structure of the data and organize documents into different categories. Examples of document analysis involve categorizing news under different topics, grouping users feedback under different product features, and an initial step for various opinion mining tasks.

In topic modeling, text clustering is generally applied using a probabilistic method to discover different topics of the document. These probabilistic methods can also be applied to discover a cluster of words or sentences, which is then further used in generating a document summary.

Text clustering is not limited only to the previously mentioned applications, it can be applied in many different applications which involve text data.

\subsection{Text Representation}

Generally, text data is represented using Bag Of Words (BOW). Other approaches for text representations include phrases, n-grams, bag of concepts, and hybrid approaches.

In the bag of words model, documents are like bags that have different words and the words can occur multiple times in a document. In a mathematical context, a bag is a multi-set which allows duplicate elements. For example $B=\{a, b, b, b, c, c\}$ is a bag containing $\mathrm{a}, \mathrm{b}$ and $\mathrm{c}$. In a bag, the order of the element is not important. A bag can also be represented as a vector. For example $V=\langle 1,3,2\rangle$ is a vector representation of the bag $B$. Likewise, documents can be represented as vectors based on the word frequencies. The document representation where documents are represented as vectors is generally formed in Vector Space Model (VSM) [115].

The bag of words model is widely applied in various fields which require text analysis e.g., information retrieval, clustering, classifications etc. Although the bag of words model is commonly used, it has many limita- 
tions. The following are the cases which shows the limitations of the bag of words representation.

- Synonymy: Two or more words might be synonyms to each other. For example the synonyms for the word dog can be a puppy, doggy, pooch, pup, hound etc. However, the bag of words model would treat them as separate features.

- Polysemy: A word can be ambiguous i.e. a word can have more than one meaning. The meaning of the word is usually determined by the context or the sentence of the word. For example the word jaguar could mean car, cat, fender etc. The bag of words approach will represent all different meanings (or features) as one feature and will ignore the context of the word.

- Interconnectedness: The bag of words model assumes all words are independent of each other. In real life, the words are interconnected with each other and different combinations of the words might produce different meanings of the sentences. This is a general issue, which involves both synonyms and polysemy of the words to discover the relatedness of two or more words. For example the connection between the word bank and the word manager or the connection between the word bank and the river. Another example of the interconnectedness can be seen in the words dark horse.

The use of phrases or n-grams to represent text is also very common in literature. It is often argued that the use of phrases or n-grams can reduce the ambiguity in text data [32]. For example, the word jaguar is an ambiguous word and it is not clear whether it means the car, the animal or the musical instrument. However, the phrases jaguar car, jaguar cat or jaguar guitar clarify the meaning of the word jaguar.

In phrase based text representation, we assume that the two segments of text or documents are more similar if they share the same phrases instead of words. Another similar text representation of text is based on 
word co-occurrence i.e. representing text using word pairs occurring together in the same sentence. Phrases are also referred as n-grams where the value of $n$ specifies the number of words in a phrase.

Text representation using phrases is widely used in different text mining fields $[3,97,4]$. In text clustering, phrases can be used to represent features $[64,60]$ and the cluster labels $[147,60,148,116,10]$ or both.

The use of phrases improves the quality of clusters and suggests that words are not the ideal features for text representation. Phrases are relatively better in capturing the semantics and avoiding the ambiguity of the documents than words. However, phrases consist of a sequence of words, they do not completely remove the ambiguity in the documents. For example, the phrase 'wild card' can refer to the name of a movie, a special character or a person in a reality shows. Another example can be the phrase 'access point' which may refer to the device of a computer network or may refer to a rocky point on the Anvers Island of Antarctica. Although, the use of phrases reduces the number of features, phrases in different documents are less likely to match than words. Hence, phrases result in much more sparse data than words [32].

Another text representation is a concept based representation. Concepts are considered to be unambiguous and each represents a unique meaning. In a concept based representation, the synonyms of a word are considered as the same feature and the different meanings or senses of one word is considered as different features. For example, the word car and vehicle are considered as one feature and the multiple senses of the word jaguar are considered as multiple features.

Lastly, text can be represented using the topics or context of the text segments or documents in text data. In other words, a document is represented by its topic or most prominent keywords. A document can have more than one topic and the documents are clustered by considering the number of shared topics of the documents. 


\subsection{Similarity Measures}

A clustering method is heavily dependent upon a similarity measure. A similarity measure determines how far or close the objects are from each other. A clustering method generally tries to form groups of objects that are close to each other. Generally, the clustering methods use pre-computed similarity matrix of all pairs of documents or compute a similarity between the documents while forming the clusters.

Computing similarities is not only the critical part of a clustering method, but also the most computation-intensive part. For a few thousands documents, the number of similarity computation can be in millions. The overall performance of the clustering algorithm can be largely degraded if one similarity measure takes more time than the other.

A very well organized survey about the similarity measures can be seen in [57]. However, the most popular and commonly used similarity measures are the cosine similarity measure and the euclidean distance $[115,69]$. The cosine similarity measure computes the similarity between two documents by considering the cosine value of the angle between two feature vectors of documents using VSM in a high-dimensional space. Let $D=\left\{d_{1}, d_{2}, d_{3}, \ldots d_{n}\right\}$ be a collection of documents and $T=\left\{t_{1}, t 2, t 3, \ldots t_{m}\right\}$ be the terms in documents. Then the cosine similarity between document $d_{i}$ and $d_{j}$ can be calculated as

$$
\operatorname{cosine}\left(d_{i}, d_{j}\right)=\frac{\sum_{t=1}^{m} f\left(d_{i t}\right) \cdot f\left(d_{j t}\right)}{\sqrt{\sum_{t=1}^{m} f\left(d_{i t}\right)^{2}} \cdot \sqrt{\sum_{t=1}^{m} f\left(d_{j t}\right)^{2}}}
$$

where $f\left(d_{i t}\right)$ and $f\left(d_{j t}\right)$ specify the frequency of the term $t$ in document $i$ and $j$ respectively.

\subsection{Clustering Methods}

Cluster Analysis or Clustering can be defined as grouping a set of similar objects into groups without any prior information [76]. Clustering meth- 
ods are desired to find distinct groups of similar objects in the data. Clustering is categorized as unsupervised learning and can be viewed as an optimization problem where the goal is to maximize some criterion measuring the quality of the clusters given some similarity measures.

Text clustering generally refers to clustering methods which provide groups of similar documents. These clustering methods can be broadly categorized based on their search approaches to the clustering problem, their cluster structures and their cluster memberships. The following are the main ways to categorize them.

- Agglomerative or Divisive Agglomerative clustering methods follow a bottom-up approach for forming a set of clusters. Initially all documents are considered as clusters and then iteratively the clusters are merged based on a similarity threshold until a stopping criterion is fulfilled. In contrast, the divisive clustering methods follow a topdown approach, where all the documents are initially considered to be in one big cluster and then the cluster is iteratively divided into smaller clusters until a stopping criterion is met.

- Hierarchical or Partition Hierarchical clustering methods generate a hierarchy of clusters, where documents are clustered into a tree structure and have parent child relationships [74]. The partition clustering methods produce a flat set of clusters [91].

- Hard or Soft Hard clustering methods restrict documents to only one cluster. In contrast, soft clustering methods allow partial memberships of the documents to the clusters and they are often categorized as fuzzy clustering methods.

There are thousands of clustering methods and covering all of them is out of the scope of this thesis. However, the following are the most popular clustering methods for text clustering. 


\subsection{Agglomerative Hierarchical Clustering (AHC)}

In Agglomerative Hierarchical Clustering (AHC), all the documents are initially considered as based clusters and then using a bottom up approach clusters are merged iteratively until all documents are merged into one big cluster. There are three common cluster merging techniques: 1) single link 2) complete link and 3) average link.

In single link the clusters are merged based on the closest pairs of documents that do not belong to the same cluster. In complete link, the clusters are merged based on the farthest pairs of documents that do not belong to the same cluster. In average link clusters are merged based on the middle (or average) pairs of documents that do not belong to the same cluster.

\subsection{Centroid Based Clustering Methods}

A typical centroid based clustering methods is k-mean clustering algorithm [91]. K-means algorithm first performs an initialization step to randomly partition the data into $k$ different clusters. Then the k-mean algorithm performs an assignment and an update step in a loop until a specific condition is achieved ${ }^{1}$. In the assignment step, documents are assigned to their nearest cluster by computing the euclidean distance between a document and a cluster mean. A cluster mean is usually a hypothetical document or a vector based on the average distances of all the documents in a cluster. In the update step, all mean points of clusters are updated.

For $n$ number of documents $\left\{d_{1}, d_{2}, d_{3}, \ldots, d_{n}\right\}$ in a dataset, the k-mean algorithm will try to minimize within-cluster sum of squares (WCSS) of $k$ clusters by formulating the following objective function:

\footnotetext{
${ }^{1}$ a stopping criteria of the algorithm, for example no change in mean values of clusters or the specified number of iteration
} 


$$
\underset{C}{\operatorname{argmin}} \sum_{i=1}^{k} \sum_{d \in c_{i}}\left\|d-\mu_{i}\right\|^{2}
$$

where $\mu_{i}$ is a hypothetical documents based on the average distances of all the documents in cluster $c_{i}$.

\subsection{Search Result Clustering}

Search result clustering aims to improve searching for information on the internet. Instead of simply listing search results of a query as a list of pages, clustering search engines present the search results as clusters of similar web pages for users to browse $[7,65]$. It is desirable for the clusters to represent different meanings of the user query, hence lexical ambiguity and polysemy issues are given a special consideration.

In search result clustering, the input is generally a list of search results. These search results consist of a URL, a title, and a snippet (a description or summary) of a web page.

The output of a search result clustering method is a set of clusters and cluster labels representing different clusters. A user typically selects a cluster label to view a list of search results related to that specific cluster label.

Search result clustering is considered to be a special case of document clustering because of its following unique challenges:

- Meaningful cluster labels

The search result clustering methods need to address the polysemy issue and be able to produce semantically meaningful cluster labels.

Traditional clustering methods generally use common keywords of the web pages in a cluster as a cluster label. However, the common keywords might not be meaningful or represent all the web pages in a cluster. 
- Computational efficiency

Since fast retrieval is one of the primary concerns in a web search, search result clustering methods are desired to have a quick response time. Generally, an online clustering approach is encouraged in developing a search result clustering methods.

\section{- Limited data}

Unlike document clustering, limited data is available for search result clustering methods. Generally, the data consists of a URL, a title and a small description or snippet of a web page. The limited amount of data makes a clustering task more difficult because of the unavailability of the rich features such as hyperlinks, meta information, markups and other contextual information.

- Unknown number of clusters

Traditional clustering methods avoid having to determine the number of clusters by predefining the number of clusters. However, in search clustering methods, different sizes and number of clusters can result from different user queries. Therefore, it is important for search clustering methods to have a mechanism which can determine the number of clusters in the data.

- Overlapping clusters

It is a natural phenomena that a document can be organized under multiple topics, therefore it is often desired for search result clustering methods to have overlapping clusters. The overlapping clusters facilitates in finding a document using multiple path or browsing through multiple clusters.

Further details of existing search result clustering methods that address some of these challenges and limitations are provided in chapter 4 . 


\subsection{Subspace Clustering}

Subspace clustering methods are widely applied when the number of features is very large. They try to group similar objects using a subset of features (i.e. subspace) instead of all features. In subspace clustering, each cluster represents a set of objects clustered according to a subspace of features. As stated in introduction chapter, subspace clustering methods can be further categorized into two main sub-categories: hard subspace clustering and soft subspace clustering.

\subsubsection{Hard Subspace Clustering}

In hard subspace clustering methods, the feature space is divided into different subspaces where each feature is either present or absent in a subspace. Hard subspace clustering methods can be further categorized by their search approach i.e. bottom-up and top-down. Examples of bottomup hard subspace clustering methods are CLIQUE [5], ENCLUS [27], MAFIA [56] and FINDIT [139]. Examples of top-down hard subspace clustering methods are PROCLUS [1], ORCLUS [2] and $\delta$-Clusters [144].

\subsubsection{Soft Subspace Clustering}

In soft subspace clustering, each feature is assigned a different weight for different subspaces. Hence some proportion of a feature is present in all subspaces. In the clustering process, features that have higher weight values in a subspace contribute more to form a cluster than the features that have lower weights. Generally, soft subspace clustering methods employ variable weighting schemes and iteratively update the feature weights in the clustering process.

Variable weighting schemes are widely applied in data mining $[37,98$, $34,35,92]$. Some of the variable weighting methods, such as k-means type variable weighting, can be extended to develop soft subspace clustering 
algorithms [50, 23, 78, 51, 40, 48].

The main limitation of these feature weighting schemes is that they ignore the semantic aspect of the text. Further details about recent soft subspace clustering methods and their limitations are provided in chapter 5 .

\subsection{Clustering Ensembles}

A key challenge in clustering is that it is possible to have more than one good solution. In the last 50 years, thousands of different clustering methods have been proposed. In recent years, researchers have focused on getting better results by utilizing the fusion of different clustering methods. Clustering methods that try to combine different clustering solutions are commonly referred as Clustering Ensembles [129].

Clustering ensemble methods use a two step clustering process: step 1 generates candidate clustering solutions, and step 2 constructs a single candidate clustering solution from the generated candidate clustering solutions.

There are different variations for clustering ensembles, but median partition based clustering ensembles is the best approach so far for step 2 of the clustering ensembles [9]. The median partition approach forms a final solution by selecting a single candidate clustering solution from the set of candidate clustering solutions. A common way of selecting the final clustering solution is to pick the candidate clustering solution that has a maximum average similarity to all generated candidate clustering solutions.

Existing clustering ensemble methods include co-association matrix based methods [47], Bayesian approaches [136], hyper-graph partitioning $[119,53]$, mixture models $[125,124]$ and evolutionary approach $[145,87]$. Apart from rare methods like [66] and [67], many of the above mentioned methods only select from candidate clustering solutions and are not able to 
construct new solutions out of the candidates. For example, by selecting some clusters from one candidate clustering solution and other clusters from another candidate clustering solution. This means that, clusters that went undetected in initial steps can never be present in final solutions [62]. Furthermore, many of the methods follow a single objective approach and implement conflicting criteria as a single function, which is argued to be a bad practice in [62].

These limitations of clustering ensembles mean that they are not able to explore the full potential of clustering ensembles. Recent clustering ensemble methods are further discussed in chapter 6 .

\subsection{Clustering as an Optimization Problem}

Clustering problems are optimization problems, the goal of clustering is to maximize some criterion measuring the quality of the clusters given some similarity measure. Real life optimization problems are mostly multi-objective in nature and often have conflicting objectives. The goal of multi-objective optimization is to search for a set of solutions that optimize a number of functions along with satisfying some constraints. These solutions are often called Pareto optimal and their plotted form provides Pareto front. The solutions are called nondominated if they are on Pareto front. Nondominated solutions mean that there is no other feasible solutions which will provide better results on one objective without affecting another.

There are many techniques for multi-objective optimization problems, however only a few of them are not sensitive to the continuity and shape features of the Pareto front. Evolutionary Algorithms are considered to be very useful for multi-objective optimization problems because they are able to work with all types of Pareto fronts. Furthermore they can find multiple Pareto optimal solutions in single iteration of the algorithm and they can provide good approximation of the true Pareto front [28].

Generally, clustering methods are formulated as single objective prob- 
lems and optimize only one criterion function. However, it would be better to formulate clustering as a multi-objective problem and to use multiple criterion functions through ensemble methods $[89,119]$ or using multiobjective optimization algorithms $[62,81]$.

\subsection{Evolutionary algorithms for optimization prob- lems}

Evolutionary algorithms are search and optimization techniques inspired by biological evolution [43]. There are numbers of clustering ensemble methods which uses evolutionary algorithms to perform the clustering. Initial candidate clustering solutions in clustering ensemble are represented as initial population and then at each iteration selection, crossover (or recombination) and mutation are performed to generate a new population (a set of new candidate clustering solutions). The iteration is usually terminated by a fixed limit for maximum number of iterations.

Generally the set of candidate clustering solutions (also called individuals) are randomly generated in the initial population. A fitness function, which is based on the objective function and constraints, evaluates each individual (candidate clustering solution) to determine its fitness value, based on the quality of clusters in a clustering solution.

Traditional clustering methods based on evolutionary algorithms are mostly single objective. However, recent trend for clustering methods based on evolutionary algorithms are generally formulated as multi-objective optimization problem.

The main aim of Multi-objective Evolutionary Algorithm (MOEA) is to approximate the true Pareto front as accurate as possible. Therefore, identifying and keeping the non-dominated solutions in different iterations along with preserving diversity becomes an important factor for the algorithm. MOEAs generally include a dominance based ranking process in 
selection the step along with an external archive (elitism) to keep the nondominated solutions that are found during the number of iterations.

Chapter 6 and 7 show how multi-objective evolutionary algorithms can be used effectively for clustering.

\subsection{Multi-View Clustering}

Documents have rich features which are distinct from each other, different multi-view learning methods can be used to improve the clustering of a text data. These learning methods can be broadly classified into four groups: co-training, co-regularization, multiple kernel learning and subspace learning.

Co-training is one of the pioneer method proposed by Blum and Michell in 1998 [17] for multi-view learning and Bickel in 2004 [13] used co-training to develop the first multi-view clustering method. Bickel used the cotraining method by considering term frequency as one view of a web document and hyperlinks as the second view of the documents and applied traditional clustering algorithms such as k-means, hierarchical clustering and EM to improve the quality of clustering solution. Other co-training based methods include multi-view spectral clustering proposed by Kumar and Daume [84] and co-EM proposed by Nigam et al., [106].

Co-regularization based methods formulate an objective function to minimize a disagreement between multiple views. Generally, the objective function is based on the graph Laplacians of all views in the data and a regularization (on eigenvectors of the Laplacians) is formed in such a way that the cluster structure remain consistent. Examples of such methods are multi-view Laplacian SVM [120], sparse multi-view SVM [122], and two co-regularization based multi-view clustering methods proposed by Kumar et al [85].

Multiple kernel learning tries to achieve a good generalization, however, can also be applied in learning from multiple views. Since multiple 
kernels correspond to multiple views, combining kernels (in a linear or non-linear way) usually produce good results. Examples of such methods are [123] and [82].

Subspace learning is based on an assumption that multiple views are generated (or came from) a shared latent subspace. Two prominent examples of these methods are Canonical Correlation Analysis [24] and Kernel Canonical Correlation Analysis [6].

Other methods of multi-view clustering include convex mixture model based multi-view clustering proposed by Tzortzis and Likas [127] and a multi-view affinity matrix based multi-view clustering proposed by de Sa et al. [33].

The literature of multi-view learning and clustering methods overlaps with clustering ensemble and subspace clustering. Further details of multiview clustering are provided in upcoming chapters.

\subsection{Summary}

Text clustering facilitates the discovery of different groups of similar text items in a data set. It can be applied in almost all domains which involve textual data. Text clustering requires a preprocessing step and a notion of similarity to perform clustering.

The preprocessing step transforms the textual data into measurable features. The preprocessing steps generally use a bag of words approach for representing the data in a vector space model. The bag of words approach provides a very basic document representation and rich features of the text are not considered. Other text representation approaches which are better than the bag of words approach include phrase based representation, ngrams and concept/topic-based text representations. However, addressing lexical ambiguity and rich features in a text, especially in the case of clustering is generally considered to be a challenging task.

The current literature on text clustering describes a range of different 
text clustering methods. Some of these clustering methods are designed for specific tasks, for example clustering results of search engines for a given user query, while others are designed for general purpose tasks such as document analysis. In both cases, text data has certain rich features. However, in specific clustering tasks such as clustering search results, there are additional rich features. In general, existing text clustering methods can be improved by utilizing rich features of text data.

Subspace clustering is a common approach in general purpose text clustering. It is different from traditional clustering methods such as hierarchical and centroid clustering methods. Subspace clustering is further divided into hard and soft subspace clustering.

A recent trend in text clustering has shifted to clustering ensembles, which generally formulate clustering as a single or multi-objective optimization problem. In both cases, a clustering ensembles method firstly generates multiple candidate clustering solutions and then derives a better clustering solution. Since evolutionary algorithms are widely applied to solve multi-objective optimization problems, recent researchers encourage to develop clustering ensemble methods based on multi-objective evolutionary algorithms.

We had observed that the current literature on subspace clustering and clustering ensembles overlaps with Multi-view clustering methods. Also different rich features can be exploited to generate multiple views. The upcoming chapters provide further details of the most relevant text clustering methods for this thesis along with their limitations and our new clustering methods. 


\section{Chapter 3}

\section{Evaluation of Clustering Algorithms}

This thesis employed empirical research methods to conduct the research and achieve the goals of the thesis. The thesis hypothesis was to investigate whether exploiting the richness of text will improve the clustering performance of text data. In order to test the hypothesis, the research work performed controlled experiments. These experiments include different tasks such as developing new algorithms for clustering text data, running these algorithms to get the results, evaluating the validity of the results, comparing the results of new clustering methods with state-of-theart clustering methods and performing the statistical test.

The computer used in all the experiments performed in this research work was standalone workstation having Linux (64 bit) with Intel(R) Core(TM) i7-3770 CPU @ 3.40GHZ, 8GB RAM and 1TB HD. The code for the algorithm was written in Java using Lingpipe ${ }^{1}$, Wikiminer[96] and Weka ${ }^{2}$ toolkits. Some of the code was also written in Matlab. Specifics of the parameter settings for the newly developed clustering methods are described along with them in upcoming chapters. This chapter describes the

\footnotetext{
${ }^{1}$ http://alias-i.com/lingpipe/

${ }^{2}$ http: //www.cs.waikato.ac.nz/ml/weka
} 
datasets and the evaluation methodology of the research work.

\subsection{Datasets}

In order to compare with other state-of-art clustering methods, our experiments were designed to use publicly available text datasets. These datasets are commonly used in the literature for evaluating clustering methods. The details of the datasets used in this thesis are as follows:

1. $\mathrm{AMBIENT}^{3}[21]$ is generated from Wikipedia with a total of 4400 documents, i.e. 100 documents for each of 44 ambiguous queries. The documents were gathered from the Yahoo search engine and were assigned to different clusters (ground truth). This dataset consists of single word queries and the total number of clusters were different for each individual query.

2. MORESQUE ${ }^{4}$ [101] is an extension of AMBIENT dataset with a total of 11400 documents i.e. 100 documents for each of 114 ambiguous queries. Similar to AMBIENT, this dataset is also generated from Wikipedia and the total numbers of clusters vary from query to query. However, MORESQUE has queries containing more than one word and is more difficult for finding clusters.

3. ODP-239 ${ }^{5}$ [22] is generated from Open Directory Project with a total of 23900 documents i.e. 100 web documents for each of 239 ambiguous queries. This dataset has more ambiguous queries than AMBIENT and MORESQUE. Clusters of this dataset are very hard to distinguish as they often have similar documents, which makes this dataset more complex compared to AMBIENT and MORESQUE.

\footnotetext{
${ }^{3}$ http://credo.fub.it/ambient/

${ }^{4}$ http://lcl.uniromal.it/moresque/

${ }^{5}$ http://credo.fub.it/odp239/
} 
4. WebKB2 ${ }^{6}$ dataset contains 1051 webpages from Cora, Texas, Washington and Wisconsin universities. The WebKB2 dataset labels the webpages into two categories, course and non-course. We divided the dataset according to the universities and constructed four datasets WebKB2-Cornell, WebKB2-Texas, WebKB2-Washington and WebKB2Wisconsin. These four datasets were then pre-processed by applying tokenization and lemmatization on each web page. We then generated two views using Term Frequency Inverse Document Frequency (TFIDF) considering only terms of the webpage and using simple Term Frequency (TF) considering only hypertext of the inlinks in the webpage.

5. WebKB4 ${ }^{7}$ dataset contains 887 webpages from the Cora, Texas, Washington and Wisconsin universities. The webpages were required to be clustered into course, faculty, student, project and staff categories. After pre-processing, we constructed two views, a binary vector of terms, which specified whether the term was present in the document or not, and the Term Frequency considering the hypertext of the inlinks in the webpages.

6. Citeseer ${ }^{8}$ dataset contains 3312 articles from the citeseer publication database. These articles were required to be clustered into six groups (Agents, AI, DB, IR, ML, HCI). After pre-processing, we constructed two views: a binary vector of terms and Term Frequency of the citations.

7. Cora ${ }^{9}$ dataset contains 2708 articles related to machine learning. These articles were required to be clustered into seven groups (Case Base, Genetic Algorithms, Neural Networks, Probabilistic Methods, Rein-

\footnotetext{
${ }^{6}$ http: //www.cs.cmu.edu/ webkb/

${ }^{7}$ http: //www.cs. cmu. edu/ webkb/

${ }^{8}$ http: //www.cs.umd.edu/ sen/lbc-proj/data/citeseer.tgz

${ }^{9}$ http: //www.cs.umd.edu/ sen/lbc-proj/data/cora.tgz
} 
forcement Learning, Rule Learning and Theory). After pre-processing, we constructed two views: a binary vector of terms and Term Frequency of the citations.

\subsection{Evaluation Methodology}

Evaluation methodology of this thesis consists of comparing the quality of the newly developed clustering methods with the quality of the state-ofthe-art clustering methods. The quality is hard to define and is generally understood as compactness of a cluster and distinctiveness of different clusters in a clustering method. A cluster with high intra-cluster similarity indicates compactness and low inter-cluster similarity between two clusters indicate distinctiveness of the clusters. A clustering method which has highest intra-cluster similarity for all clusters and the lowest inter-cluster similarity within all clusters is considered the best clustering method.

An alternative approach for measuring the quality of produced clusters of a clustering method is to compare the clusters in a clustering method with a gold standard of benchmark datasets. The gold standard clustering (also called ideal clustering) is generally made by domain experts (human judges). A clustering method is compared with gold standard using an external criterion that provides a quantitative score (generally ranges from 0-1). This score determines how close the clustering is from the gold standard or ideal clustering. Clusters in the gold standard are also called classes in this thesis to distinguish them from clusters produced by the clustering methods.

A common misunderstanding in clustering is to think that the gold standard can be used to guide the clustering process. Note that clustering is unsupervised learning, and there is no information about the gold standard during the clustering process. The gold standard can only be used for evaluation after the clustering process is finished and a new clustering method is generated. Also the concepts and calculation of Precision and 
Recall are slightly different in evaluation of clustering methods than the literature of Information Retrieval.

Let $C=\left\{c_{1}, c_{2}, c_{3}, \ldots, c_{k}\right\}$ be a set of clusters and $\mathcal{G}=\left\{g_{1}, g_{2}, g_{3}, \ldots, g_{q}\right\}$ be a set of classes in a gold standard. $c_{i}, g_{r}$ are individual clusters and classes that represents a set of objects/documents in clustering $C$, and the gold standard $\mathcal{G}$ respectively. Then we can use the following evaluation metrics (external criterion) to evaluate the quality of clustering methods.

1. Precision: Precision ${ }^{10}$ measures the accurate assignments of the objects in a cluster by considering a corresponding cluster class of the gold standard. The correspondence is determined by the majority of the objects in a cluster, which are also grouped together in a class of the gold standard. Once the correspondence between the clusters and the classes are determined, then correct assignments of the objects in all clusters are divided by the total number of objects to compute the precision score. Formally, the precision is defined as:

$$
\operatorname{Precision}(C, \mathcal{G})=\frac{\sum_{i=1}^{k} \max _{r}\left(n_{i}^{r}\right)}{\sum_{i=1}^{k} n_{i}}
$$

where $k$ represents the total number of clusters, $n_{i}$ represents the number of documents in cluster $i$ and $n_{i}^{r}$ represents the number of documents in cluster $i$, which also belong to class $r$ of the gold standard. The value of precision ranges from 0 to 1 , where 1 means the clustering method exactly matches the ideal/gold standard. The precision is also called purity or clustering accuracy $[30,152,105]$.

2. Entropy: Entropy is used to measure the distortion or disorder in a cluster i.e members of different classes of a gold standard that should not have been grouped together in clusters [152]. Formally, the entropy is defined as:

\footnotetext{
${ }^{10}$ There are other methods for calculating precision, however, we made sure that we only use definition explained here
} 


$$
\operatorname{Entropy}(C, \mathcal{G})=\sum_{i=1}^{k} \frac{n_{i}}{n} E\left(c_{i}\right) ; \quad E\left(c_{i}\right)=-\frac{1}{\log q} \sum_{r=1}^{q} \frac{n_{i}^{r}}{n_{i}} \log \frac{n_{i}^{r}}{n_{i}}
$$

where $c_{i}$ is a cluster, $k$ is the number of clusters, $q$ is the number of classes in gold standard, $n_{i}$ is the number of documents in cluster $c_{i}$, $n_{i}^{r}$ is the number of documents in both $c_{i}$ and $g_{r}$ and $r$ ranges from 1 to $q$.

Generally the entropy ranges from 0 to 1 , where 0 means there is no distortion and each cluster is perfectly matched with one of the ideal/gold standard clusters.

3. Recall: Unlike precision and entropy, recall ${ }^{11}$ checks if all the classes were accurately covered by the clustering method. The majority of the objects in a cluster, which have the same class in the gold standard, forms a correspondence of that cluster to the class. Once each cluster formed a correspondence to a class in the gold standard, the recall computes a score based on the number of covered classes and all classes in the gold standard [30]. Formally recall is defined as:

$$
\operatorname{Recall}(C, \mathcal{G})=\frac{\sum_{r=1}^{q} \sum_{c_{i} \in C^{r}} n_{i}^{r}}{\sum_{r=1}^{q} n_{r}}
$$

where $C^{r}$ is a subset of clusters in a clustering method $C$ whose large portion of the documents belongs to class $r . n_{r}$ is the number of documents that belong to class $r, n_{i}^{r}$ is the number of documents that belongs to both $c_{i}$ and $g_{r}$ and $q$ is the number of classes.

The Recall value ranges from 0 to 1 , where 1 means all classes are accurately covered by the clustering method.

\footnotetext{
${ }^{11}$ There are other methods for calculating recall, however, we made sure that we only use definition explained here
} 
4. F-measure: F-measure ${ }^{12}$ is defined as the weighted harmonic mean of the Precision and Recall. Precision is the measure of the accuracy of a system whereas Recall is a measure for the coverage of different topics in a clustering method [30]. F-measure is defined as:

$$
F-\text { measure }=\frac{2 \text { Precision } \times \text { Recall }}{\text { Precision }+ \text { Recall }}
$$

5. Rand Index: Rand Index (RI) is widely used in literature to examine the agreement of a clustering method with the ground truth (gold standard) [70]. The RI can be computed as:

$$
R I(C, \mathcal{G})=\frac{T P+T N}{T P+F P+F N+T N}
$$

where $C$ is a clustering method and $\mathcal{G}$ is a ground truth. TP, TN, FP and FN are the total number of true positives (pairs that are in the same cluster in a $C$ and $G$ ), true negatives (pairs of documents that are in different clusters in both $C$ and $G$ ), false positive (pairs of documents that are in different clusters in $\mathcal{G}$ but in the same cluster in $C$ ) and false negatives (pairs of documents that are in different clusters in $C$ but in the same cluster in $\mathcal{G}$ ) respectively.

6. Normal Mutual Information (NMI): NMI also measures the quality of a clustering method (overall structural quality) by matching how close the clusters are to the classes in the gold standard. However, it differs in a sense that it is not biased towards high numbers of clusters [154]. The NMI can be computed in different ways. In this thesis, we used an entropy based geometrical mean. Formally the NMI is defined as:

$$
\mathrm{NMI}=\frac{\sum_{i=1}^{k} \sum_{r=1}^{q} n_{i}^{r} \log \left(\frac{n \cdot n_{i}^{r}}{n_{i} \cdot n_{r}}\right)}{\sqrt{\left(\sum_{i=1}^{k} n_{i} \log \frac{n_{i}}{n}\right)\left(\sum_{r=1}^{q} n_{r} \log \frac{n_{r}}{n}\right)}}
$$

\footnotetext{
${ }^{12}$ There are other methods for calculating f-measure, however, we made sure that we only use definition explained here
} 
where $q$ is the total number of classes in the gold standard, $k$ is the total number of clusters and $n$ is the total number of documents in a dataset. $n_{r}$ and $n_{i}$ represents the number of documents in class $r$ of the gold standard and in cluster $i$ of the clustering method respectively. $n_{i}^{r}$ represents the number of documents that are common in both class $r$ and cluster $i$. 


\section{Chapter 4}

\section{Search Result Clustering}

\subsection{Introduction}

The most important job of search engines is to display the results that matches a user query in such a way that users can easily find the desired information. A user query that has multiple senses is often called an ambiguous query. The user query jaguar is an ambiguous query e.g. it can mean American big cat, the British car manufacturer, an operating system by Apple, etc. Traditional search engines will return the list of ranked pages without considering the different senses of the query, leaving the user to browse through irrelevant pages. In contrast, clustering search engines such as Carrot use Search Result Clustering algorithms that group documents and provide descriptions for these groups enabling users to find their desired results in a more efficient way.

The goal of Search Result Clustering is not only to cluster search results but also to provide semantically meaningful cluster labels. A cluster label is a one-phrase description of all the documents in a cluster, and users often decide whether to browse the list of documents in a cluster by looking at the cluster label. It is a common practice to use the most common keywords shared by all the documents in a cluster as a cluster label. Documents can have common keywords that might represent either more than 
one or more senses of the user query. Therefore, cluster labels based on common keywords are not always useful to the user. Also the clusters will be more useful to the user if all the documents in a cluster represent only one particular sense of the user query.

Traditional Search Result Clustering algorithms which ignore the user query are more prone to the problems of low quality cluster and poor cluster labels. Low quality cluster is a cluster having documents that represent more than one sense of the user query and poor cluster labels are cluster labels that do not represent any senses of the user query.

In traditional clustering algorithms, the similarity between two documents is often measured based on the text representation using word frequency. Such similarity measures are regarded as syntactic measures because they only consider counts of words.

This chapter presents a new algorithm Query Sense Clustering (QSC) that exploits the user query and uses both syntactic and semantic features (topics) of a document for the clustering solution.

The chapter is organized as follows: section 4.2 highlights the related work; section 4.3 discusses the representation and similarity measures of the documents and the query senses; section 4.4 describes the algorithm; section 4.5 focuses on the evaluation and the analysis of the results and section 4.6 provides a brief summary of the chapter.

\subsection{Existing SRC Methods}

Search Result Clustering (SRC) methods can be classified into three categories: data-centric, description-aware and description-centric [19].

The data-centric category contains traditional clustering algorithms (hierarchical, partitioning) and the focus is on the clustering process. The Scatter/Gather algorithm $[65,111]$ is the pioneer example of the datacentric category. Other prominent examples are WebCat [54], AISearch [117], LASSI [90], TRSC [104] and Link-based clustering [151]. The main 
drawback of this category is the poor cluster labels which are often generated from the text and are often meaningless.

The description-aware methods carefully select one or more features to construct meaningful cluster labels. Suffix Tree Clustering (STC) $[147,146]$ was the first algorithm that used suffix trees to build cluster labels and performed clustering on search results. Later it was improved by fixing the scalability issue $[18,93]$, implementing a better scoring function (ESTC) [30] and approximating sentences for better document similarity (SnakeT) [45]. The issue with description-aware methods is that the cluster labeling procedure dominates the clustering process and the overall quality of the clusters is compromised.

The description-centric methods are specialized clustering methods that not only focus on cluster labels but also try to provide quality clusters. Examples in this category include LINGO [107], DisCover [86], CREDO [20], KeySRC [10] and STHAC [140]. Our algorithm QSC also belongs to this category.

The user query was first used by QDC [31] to guide the clustering process. Later EQDC [109] improved the QDC method to handle key phrases. However it was based on ESTC [30] and does not consider the query senses explicitly.

Apart from the above, word sense induction based methods such as Curvature [41], SquaT++ [101, 39], B-MST [38], HyperLex [130], Chinese Whispers [14] are related to our method QSC because they use query senses. They all identify multiple senses of the query by applying graph based word co-occurrence techniques. Our method QSC identifies query senses from Wikipedia and is more similar to SRCluster [95]. However both word sense induction based methods and SRCluster use syntactic features of the document whereas in QSC, we use both syntactic and semantic features of the document to construct the similarity measure. The comparison results of our algorithm with these algorithms are given in section 4.5 . 


\subsection{Representation and Similarity Measure}

This work uses query senses to generate initial clusters and then uses a new document similarity measure to refine the initial clusters. The document similarity measure is based on a new document representation using both syntactic and semantic features (topics). The following subsections introduce the new document representation, the document similarity measure, the query sense representation and the sense similarity measure. The algorithm is presented in section 4.4 .

\subsubsection{Document Representation}

The traditional bag-of-words model is widely used in document clustering to represent documents in Vector Space. Terms are commonly weighted using the Term Frequency Inverse Document Frequency (TFIDF) weighting scheme [114]. A document $d$ in term-space is represented as

$$
\operatorname{Tm}(d)=\left\{t f i d f\left(t_{1}, d\right), \operatorname{tfidf}\left(t_{2}, d\right), \operatorname{tfidf}\left(t_{3}, d\right), \ldots, t f i d f\left(t_{n}, d\right)\right\}
$$

where $n$ is the total number of terms and tfidf is the tf-idf function defined as

$$
t f i d f(t, d)=t f(t, d) \times \log \frac{|D|}{d f(t)}
$$

where $t f(t, d)$ is the frequency of term $t$ in the document $d,|D|$ is the total number of documents and $d f(t)$ is the number of documents containing term $t$. A criticism of this model is that it only uses a syntactic representation of the document and ignores the semantic representations of the document. One semantic representation is based on topics representing the subjects or concepts that a document is about. If we can identify all the topics of a document, then we can represent a document as a vector in topic space with weights for each topic representing the importance of the topic to the document. We propose a new document representation in which a document $d$ containing topics $\tau_{1} \ldots \tau_{m}$ in topic-space is represented 
as

$$
T p(d)=\left\{w\left(\tau_{1}, d\right), w\left(\tau_{2}, d\right), w\left(\tau_{3}, d\right), \ldots, w\left(\tau_{m}, d\right)\right\}
$$

where $m$ is total number of topics and $w(\tau, d)$ is a weight of a topic $\tau$ in document $d$, generated using the topic detector of Wikiminer Toolkit [96].

\subsubsection{Document Similarity Measure}

The most common and well known similarity measure for comparing documents is cosine the similarity function [108]. Inspired from [126], we define the combined cosine similarity that includes semantic and syntactic features of document $d_{i}$ and $d_{j}$ as

$$
\operatorname{Sim}\left(d_{i}, d_{j}\right)=\lambda \operatorname{Cosine}\left(\operatorname{Tp}\left(d_{i}\right), \operatorname{Tp}\left(d_{j}\right)\right)+(1-\lambda) \operatorname{Cosine}\left(\operatorname{Tm}\left(d_{i}\right), \operatorname{Tm}\left(d_{j}\right)\right)
$$

where $\lambda$ is a scaling variable and the value of $\lambda$ is 0.1 based on the preliminary experiments. $T p(d)$ is the document vector in topic-space and $T m(d)$ is the document vector in term space.

We initially set the value of lambda to 0.5 (i.e equal weights) and then fine tune the value of lambda based on our results.

\subsubsection{Query Sense Representation}

We represent a query using a set of senses $S=\left\{s_{1}, s_{2}, s_{3} \ldots s_{n}\right\}$ of the query which are generated using Wikiminer [96] word disambiguation. These raw senses are filtered and noise is removed by using tokenization, stemming and stop word removal techniques. Tokens generated from these senses are mostly bi-grams such as jaguar car, sepecat jaguar, fender jaguar, mac os. Other examples of senses are panthera and south alabama jaguar football. 


\subsubsection{Sense Document Similarity Measure}

We define the similarity score between a document $d_{i}$ and a sense $s_{j}$ as a weighted sum of six different criteria:

$$
\operatorname{SimSense}\left(d_{i}, s_{j}\right)=\frac{\left|s_{j}\right|}{\left|d_{i}\right|} \sum_{k=1}^{6} w_{k} \cdot \operatorname{cmp}_{k}\left(d_{i}, s_{j}\right)
$$

The six criteria for $\mathrm{cmp}$ are calculated using three measures (exact sequence matching, partial matching and semantic matching) in two spaces (term space and the topic space) of the document $d_{i}$ for sense $s_{j}$, where $\left|d_{i}\right|$ and $\left|s_{j}\right|$ denotes the number of tokens in document $d_{i}$ and sense $s_{j}$.

The exact sequence matching counts the number of occurrences of a sense $s_{j}$ in document $d_{i}$ whereas the partial matching counts overlap of individual tokens in a sense $s_{j}$ and document $d_{i}$. The semantic matching counts the overlap of either exact tokens or the synonyms of the tokens.

The six weights are described in Table 4.1. The weights were split first for the term space and the topic space, and then adjusted later. These weights are split and adjusted based on the following observations.

- The similarity in term space is normally more useful and accurate than the similarity in the topic space. The main reason is that the topics are often very short and small in number, and a match between topic words and query senses is hard to find.

- In case of topic space, the weights of exact sequence matching, semantic matching and partial token matching are equally important to get good results. Because both the query sense and the topics are often very short and normally do not have any ambiguity, so any matching between query senses and topics including partial token matching is considered equally good.

- In term space, we give high weights to exact and semantic matching. Partial token matching in term space is given the lowest weight based on the following reasons. 
The document is more relevant if the exact sequence of the tokens in a query sense is found in the document instead of a single token. For example, if the query sense is "Rider of the lost ark" then the most relevant document should be the one which contains the exact phrase.

The query sense "Jaguar Panthera" and "Jaguar Cat" refers to the same concept, however, the "Jaguar Car" refers to another concept. Therefore, in order to get better results we should consider higher weights for exact sequence matching or semantic matching than partial token matching. For example, a document with many occurrence of "Jaguar" (and "Jaguar" only) should not be counted as more relevant.

The total sum of all the weights $(0.26+0.26+0.09+0.13+0.13+0.13)$ is equal to 1 . Please note that our weights were tuned on the training data set and the above mentioned observations were also true for the test datasets. However, for other datasets the values of the weights might need to be slightly adjusted to get better results.

Table 4.1: Weight distribution of the Sense Document Similarity Measure

\begin{tabular}{lcc}
\hline & Term Space & Topic Space \\
Exact Sequence Matching & 0.26 & 0.13 \\
Semantic Matching & 0.26 & 0.13 \\
Partial Token Matching & 0.09 & 0.13 \\
\hline
\end{tabular}

\subsection{The Algorithm}

We developed a new algorithm called QSC that uses our new document representation and similarity measures. The algorithm falls under the area 
of incremental clustering with refinement step [102]. It includes three main steps: the first step is to group all the documents according to their similarity to the different senses of the user query; the second step is to iteratively optimize clusters by relocating documents from one cluster to another cluster based on the similarity between documents; the third step is to rank the clusters based on their similarity with the user query, and to sort the documents in each cluster.

\subsubsection{Step 1: Initial Cluster Generation}

The initial clusters are formed by calculating the similarity of each document with each user query sense and assigning each document the most similar sense. Each cluster is labeled with its associated sense. Documents that are not sufficiently similar to any senses are placed in a cluster labeled general. The set of initial clusters $C$ consists of all the clusters that contain at least one document.

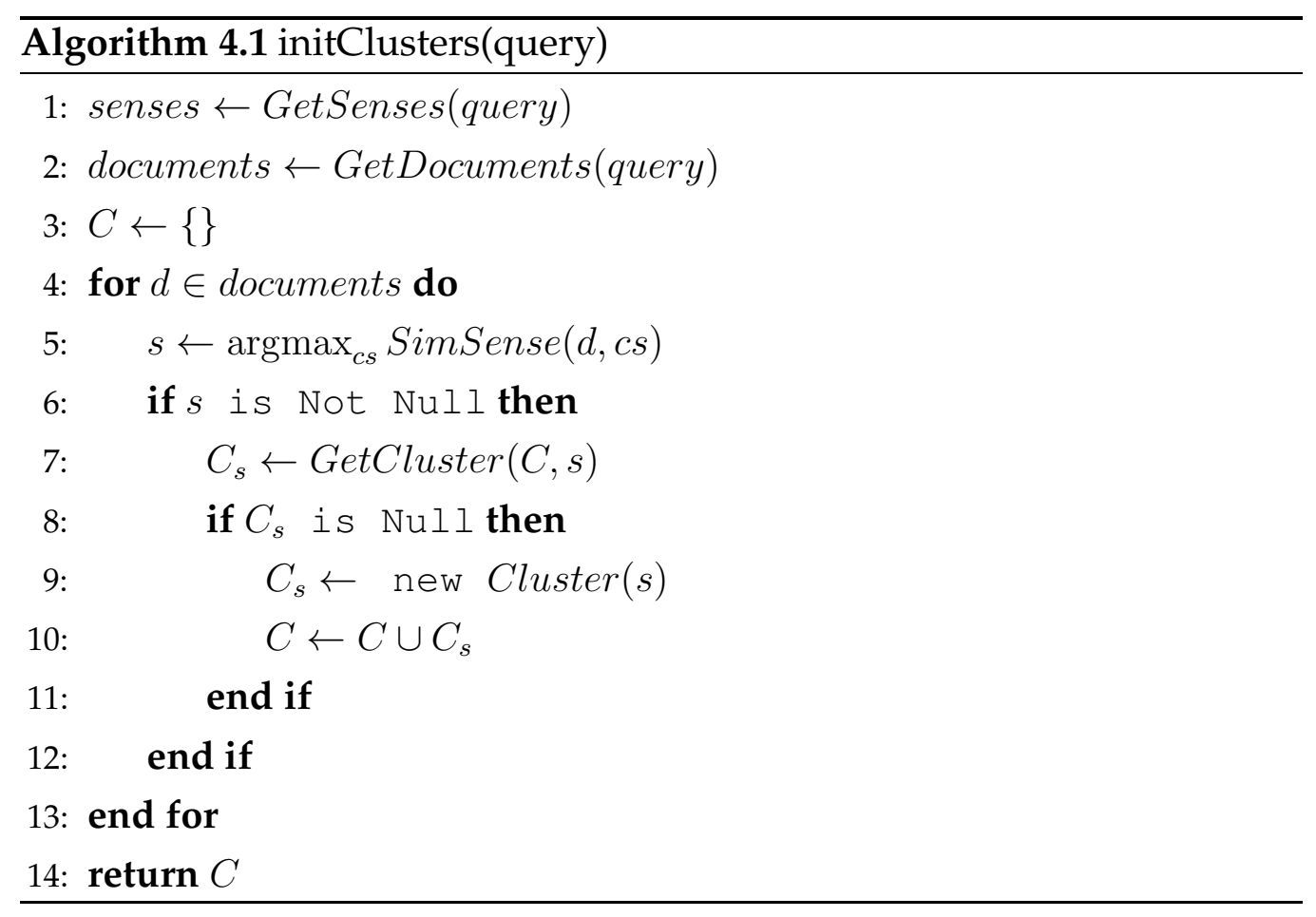


Algorithm 4.1 shows the pseudo code for generating the initial clusters. The function initClusters takes an argument query that is given by user on runtime. The function GetSenses provides all the senses of a query and the function GetDocuments provides all pre-processed documents along with their topics. The loop on Line 5 iterates over all senses and finds the most similar sense for document $d$. The function GetCluster is used to update an existing cluster that has cluster label $s$, or create a new cluster object $C_{s}$ which is added to the list of clusters $C$.

\subsubsection{Step 2: Cluster Optimization}

Initial clusters were based on the similarity between documents and the senses. Base cluster labels can provide quality labeling of clusters. However the clusters, especially the general cluster may contain a mixed group of documents that might not be similar. We developed an iterative method to reassign some documents in order to improve the cluster quality by increasing the intra-cluster coherence and the inter-cluster distinctiveness.

The pseudo code for this optimization is given in Algorithm 4.2. Just like k-means, the algorithm repeatedly attempts to relocate documents to more appropriate clusters. The algorithm terminates if it fails to make any changes, or if it reaches the predefined maximum number of iterations. The function UpdateMean updates the average similarity scores of all documents in clusters $c_{x}$ using the new similarity measure given in Equation 4.4. The function RelocateDocument removes document $d_{x}$ from cluster $c_{x}$ and adds it to another cluster $c_{y} \in C: c_{y} \neq c_{x}$ if the average similarity of the document $d_{x}$ in cluster $c_{x}$ is lower than its mean in $c_{y}$. The function Filter EmptyClusters removes all clusters with no documents. One result of this algorithm is that closely related clusters can be merged by relocating all documents from one cluster to the other. 


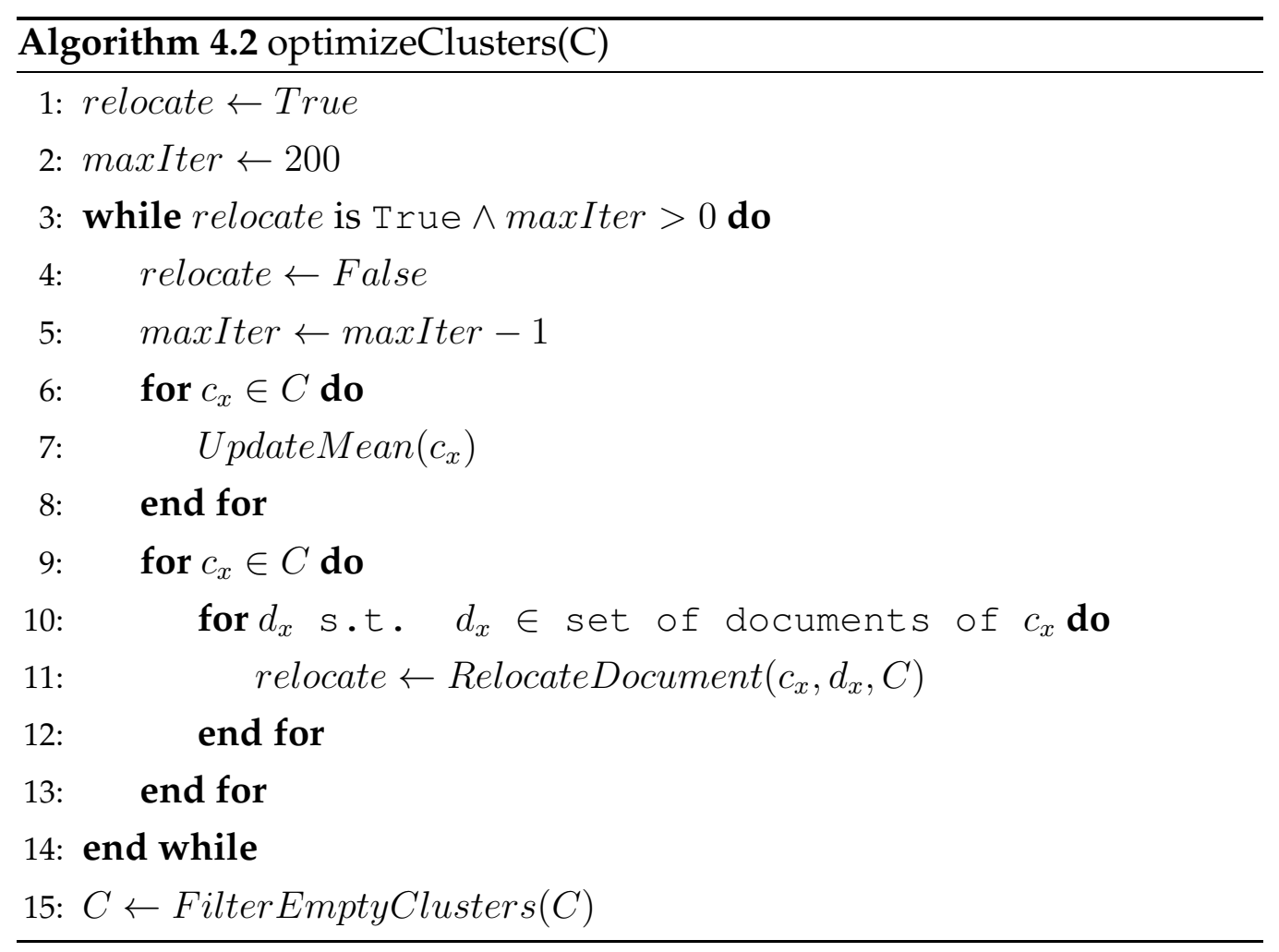




\subsubsection{Step 3: Cluster Ranking}

Users are interested in only those documents that are most closely related to the query. Therefore the ranking of clusters and documents are computed with respect to the query.

All the clusters were sorted, by calculating the relatedness score between the user query and the cluster label, using the term similarity measure WikiSim [73]. WikiSim is a Wikipedia based similarity measure that computes the relatedness between two terms. Documents in its own cluster were also sorted by calculating the similarity of a document to its mean in its own cluster. The ranked result list is then sent to the user for browsing.

\subsection{Results}

QSC was evaluated on two datasets, AMBIENT and MORESQUE interms of Purity, Entropy and F-measures which are described in chapter 3. Please note that the literature survey shows that F-measure can be calculated in different ways, but we make sure that the same implementation is used while comparing our results with previous research.

\subsubsection{Comparison 1}

This work was compared with two popular algorithms STC [146] and Lingo [107] and one recent algorithm SRCluster [95], on a subset of the AMBIENT dataset. This subset consists of 9 queries chosen by SRCluster [95]. The comparison is based on two evaluation criteria namely Entropy and Purity [152].These criteria are widely used in evaluating cluster quality and the coherence of a cluster.

Table 4.2 and 4.3 list the 9 queries of the AMBIENT dataset as chosen by the paper [95]. The values of Entropy and Purity for STC, LINGO and 
SRCluster were taken from the paper of SRCluster [95]. The values of QSC were computed on the same 9 queries to ensure fair comparison.

Table 4.2: The Values of Entropy on a subset of AMBIENT Dataset

\begin{tabular}{lcccc}
\hline & \multicolumn{4}{c}{ Entropy } \\
\cline { 2 - 5 } Query & STC & LINGO & SRCluster & QSC \\
\hline Aida & 0.210 & 0.449 & 0.103 & $\mathbf{0 . 0 1 8}$ \\
Camel & 0.142 & 0.650 & 0.032 & $\mathbf{0 . 0 1 9}$ \\
Indigo & 0.390 & 1.000 & 0.071 & $\mathbf{0 . 0 2 0}$ \\
Jaguar & 0.190 & 0.495 & 0.041 & $\mathbf{0 . 0 2 7}$ \\
Excalibur & 0.880 & 1.000 & 0.080 & $\mathbf{0 . 0 3 7}$ \\
Minotaur & 0.150 & 0.400 & 0.068 & $\mathbf{0 . 0 3 9}$ \\
Urania & 0.229 & 0.700 & 0.299 & $\mathbf{0 . 0 2 3}$ \\
Zenith & 0.245 & 1.000 & 0.047 & $\mathbf{0 . 0 1 7}$ \\
Zodiac & 0.520 & 1.000 & $\mathbf{0 . 0 0 0}$ & $\mathbf{0 . 0 0 0}$ \\
\hline
\end{tabular}

The cluster quality is determined on the basis of lower entropy and higher purity. QSC outperformed other methods in almost all queries except the query Zodiac. The query Zodiac has entropy 0 and purity 1 for both SRCluster and QSC which indicates that both methods are perfect for this query.

\subsubsection{Comparison 2}

The results on the larger dataset, which consists of all queries of AMBIENT and MORESQUE, based on purity and entropy were not given in [95]. However we found another recent paper [39] that compared nine algorithms using F1-measure on this large dataset. Therefore we compared our algorithm QSC with these nine algorithms using F1-measure 
Table 4.3: The Values of Purity on a subset of AMBIENT Dataset

\begin{tabular}{lcccc}
\hline & \multicolumn{4}{c}{ Purity } \\
\cline { 2 - 5 } Query & STC & LINGO & SRCluster & QSC \\
\hline Aida & 0.030 & 0.780 & 0.655 & $\mathbf{0 . 8 7 8}$ \\
Camel & 0.040 & 0.550 & 0.919 & $\mathbf{0 . 9 2 8}$ \\
Indigo & 0.000 & 0.400 & 0.867 & $\mathbf{0 . 9 2 5}$ \\
Jaguar & 0.290 & 0.290 & 0.888 & $\mathbf{0 . 9 2 0}$ \\
Excalibur & 0.000 & 0.090 & 0.774 & $\mathbf{0 . 8 4 0}$ \\
Minotaur & 0.220 & 0.300 & 0.760 & $\mathbf{0 . 8 7 1}$ \\
Urania & 0.140 & 0.348 & 0.782 & $\mathbf{0 . 8 8 8}$ \\
Zenith & 0.000 & 0.500 & 0.871 & $\mathbf{0 . 9 5 8}$ \\
Zodiac & 0.000 & 0.389 & $\mathbf{1 . 0 0 0}$ & $\mathbf{1 . 0 0 0}$ \\
\hline
\end{tabular}

calculated by taking the harmonic mean of precision and recall of the cluster [30]. The comparison was made between STC, LINGO, KeySRC [10], Curvature [41], SquaT++ [101, 39], B-MST [38], HyperLex [130], Chinese Whispers [14] and QSC.

Figure 4.1 shows the percentage values of F1-measure, Rand Index, Jaccad Index and Adjusted Rand Index of 10 methods on combined dataset of AMBIENT and MORESQUE taken from the paper [39] and the computed values of QSC. Clearly, QSC performed significantly better than others and have the highest values 83.62 and 82.18 of F1-measure and Rand Index respectively. QSC performed comparatively well on Adjusted Rand Index and Jaccad Index as compared to the rest of the clustering methods.

\subsubsection{Comparison 3}

This subsection compares our method with search engines in terms of information retrieval performance. The search results need to be diverse 


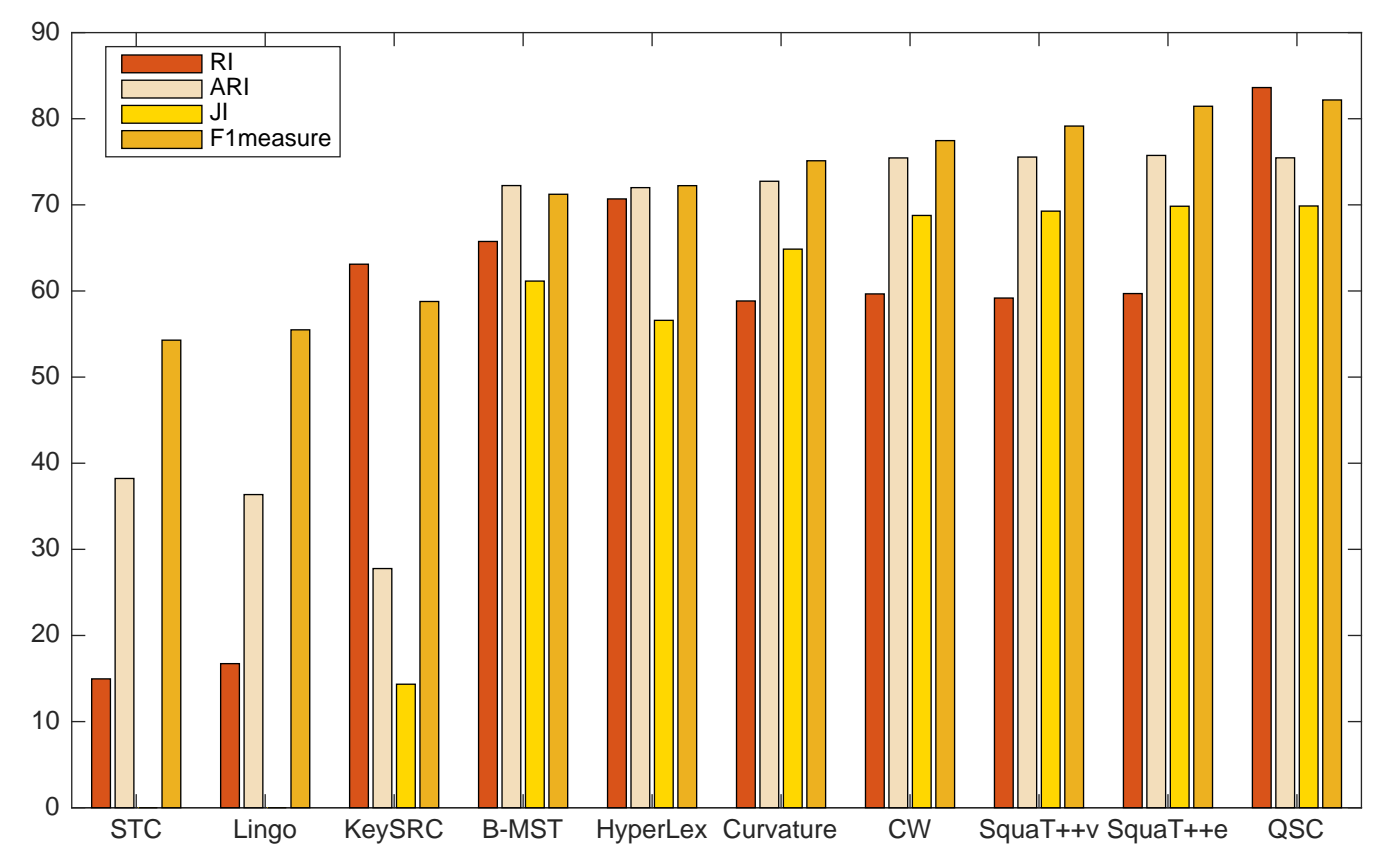

Figure 4.1: Comparison of SRC methods based on different evaluation measures

and top ranked results should represent different senses of the user query. In order to determine the diversification of this work, the search results were evaluated based on S-recall@K (Subtopic recall at rank K) and Sprecision@r (Subtopic precision at recall) [149] on the combined dataset of AMBIENT and MORESQUE. The former evaluates the performance of the system based on $K$ top-ranked results for the number of topics of query q. S-precision@r measures the ratio of subtopics covered by the minimum set of results at given recall $r$.

These two measures are used to compare search engines (Yahoo! and Essential Pages) that return ranked list of search results. The results returned by QSC were compared by flattening the clusters. The result list was formed by iterating through clusters and selecting top results. The clusters that only had one document were appended at the end to avoid noise. 


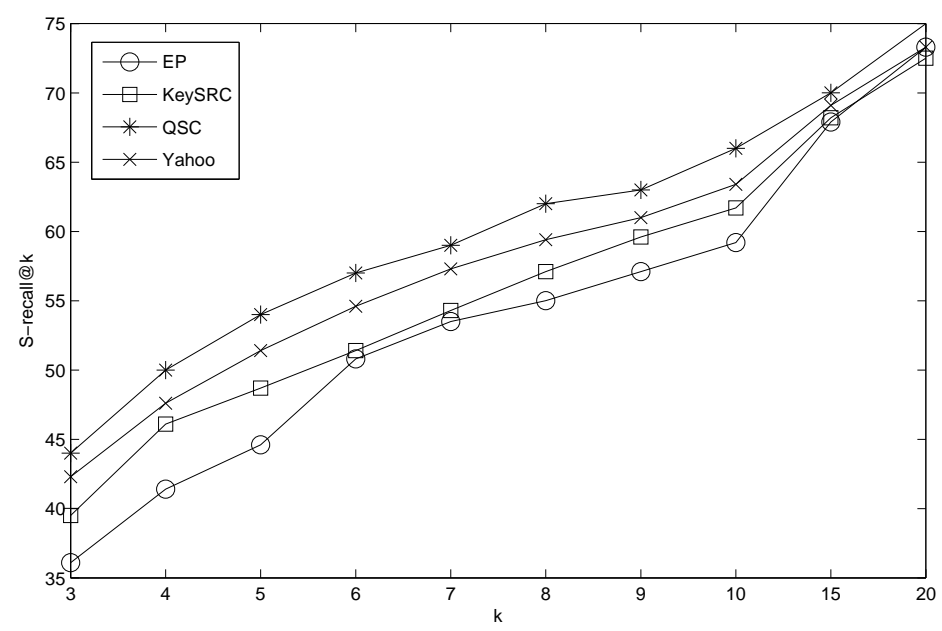

Figure 4.2: S-recall@k on all queries

Figure 4.2 and Figure 4.3 show the S-recall@k and S-precision@r respectively for search results of Yahoo, Essential Pages(EP), KeySRC and QSC. The QSC performs relatively better in terms of S-recall@k and significantly outperformed others in terms of S-precision@r for the given values of $k$ and $r$. Please note that QSC relies on yahoo to get the initial documents. These results show that QSC can improve the diversity of the top ranked results.

\subsubsection{Further Analysis}

The detailed analysis consists of three sub sections: the first discusses the cluster labels; the second discusses the processing time of QSC, and the third discusses the cluster numbers and some observations about final clusters.

\section{Cluster Label Analysis:}

The goal of the QSC algorithm is to generate a useful set of distinct clusters with informative labels. 


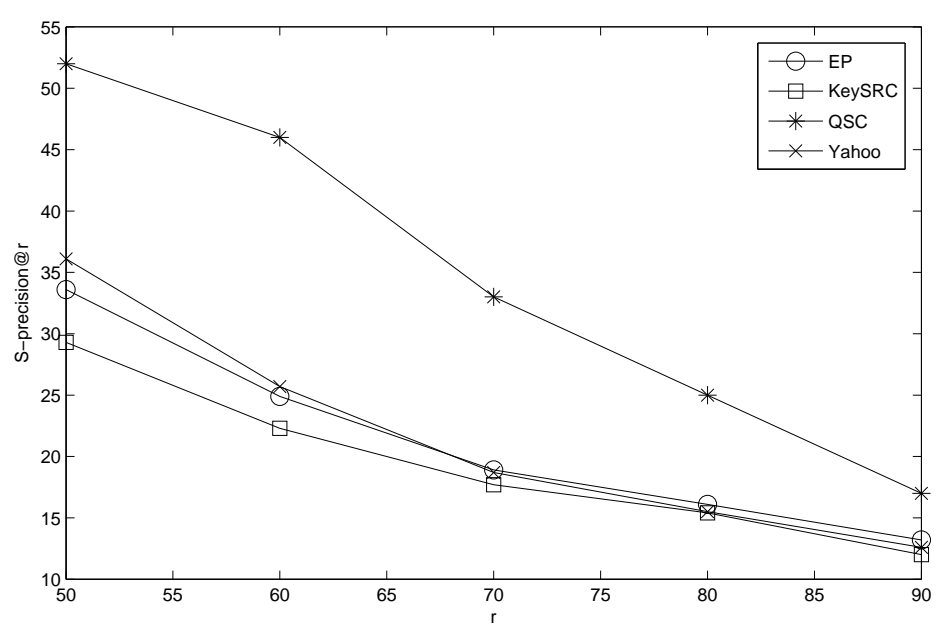

Figure 4.3: S-precision@r on all queries

Table 4.4 shows the cluster labels of the clusters generated for the query Jaguar by STC, LINGO and QSC (the cluster labels are not in ranked order). The labels for STC and LINGO were generated using the Carrot2 framework by adjusting the parameter of maximum clusters number to 8. Table 4.4 shows that the cluster labels generated by QSC provide more precise, intuitive and distinct labels than the cluster labels from STC and LINGO.

\section{Processing Time:}

QSC was evaluated on standalone workstation using Linux (64 bit) with Intel(R) Core(TM) i7-3770 CPU @ 3.40GHZ, 8GB RAM and 1TB HD. Figure 4.4 shows the processing time of all the queries in AMBIENT Dataset. The average time required for processing the query is under 1.0 second for both AMBIENT and MORESQUE datasets. Most of the queries were processed under one second with a few exceptional cases. The maximum processing time was 6.3 seconds on a query jaguar because it had 54 senses to be processed. This processing time was reduced to 1 second by eliminating overlapping senses and processing only 10 distinct senses. 
Table 4.4: Cluster labels of STC, LINGO and QSC of the query Jaguar

\begin{tabular}{lll}
\hline STC & LINGO & QSC \\
\hline Jaguar Car & Auto Show & Jaguar Car \\
S-Type, Used Jaguar & Jaguar Parts & Jaguar E-Type \\
XK, 2006 2007, Price- & Dealer Price Quotes & Jaguar XK \\
Quotes and Reviews & and- Reviews & \\
Ford Motor & Ford Motor & \\
Company- Division & Company- Division & \\
Jaguar Cars & & \\
Jaguar Panthera & Jaguar Panthera & Panthera \\
Onca & Onca & \\
Jaguar Animal & & Fender Jaguar \\
& Website of Fender & Mac OS X \\
Information & Musical- Instrument & SEPECAT Jaguar \\
New & Jaguar Video & South Alabama \\
& & Jaguar- Football \\
\hline
\end{tabular}

Strictly speaking, we cannot directly compare the processing time of algorithms due to different machines and platforms. However we would like to give indications that word sense induction based algorithms (Curvature, Squat++, B-MST, HyperLex and Chinese Whispers) need to construct the graph to identify the senses from the huge corpus, whereas QSC extract the senses from Wikipedia. Therefore the word sense induction based algorithms might require more processing time than QSC. The processing time of clustering, without considering the time spent on graph construction, for all algorithms is under 1 second except for SquaT++ al- 
gorithm. The SquaT++v and SquaT++e spent around 28 and 21 seconds respectively for clustering results as described in their paper [39].

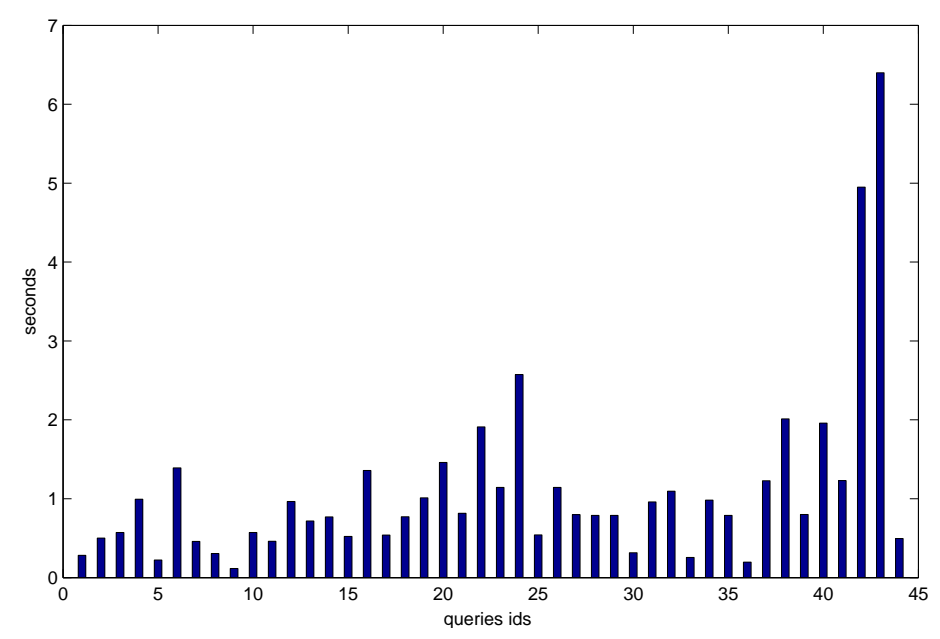

Figure 4.4: Processing Time for All Queries

\section{Cluster Analysis:}

The average number of clusters for all queries in the AMBIENT dataset was 7.84 i.e on average 7-8 clusters are formed for each query. The average number of clusters for all queries in the AMBIENT and MORESQUE datasets was 5.4. There were a few queries with a high number of clusters and the maximum number of clusters was 18 for the query Monte Carlo. In contrast the query Life on Mars just had 1 big cluster. The reason for having many clusters was the large number of distinct query senses. The query Life on Mars had very few senses and they were overlapping with each other, e.g. Life on Mars (TV series), Life on Mars (U.S TV series), and this causes single cluster for the query.

QSC provided a more fine-grained clustering solution than the gold standard (manually labeled search results). The gold standard for the query jaguar had 7 clusters but QSC solution provided 9 clusters. The three clusters jaguar car, jaguar e-type and jaguar $x k$ in QSC were sub 


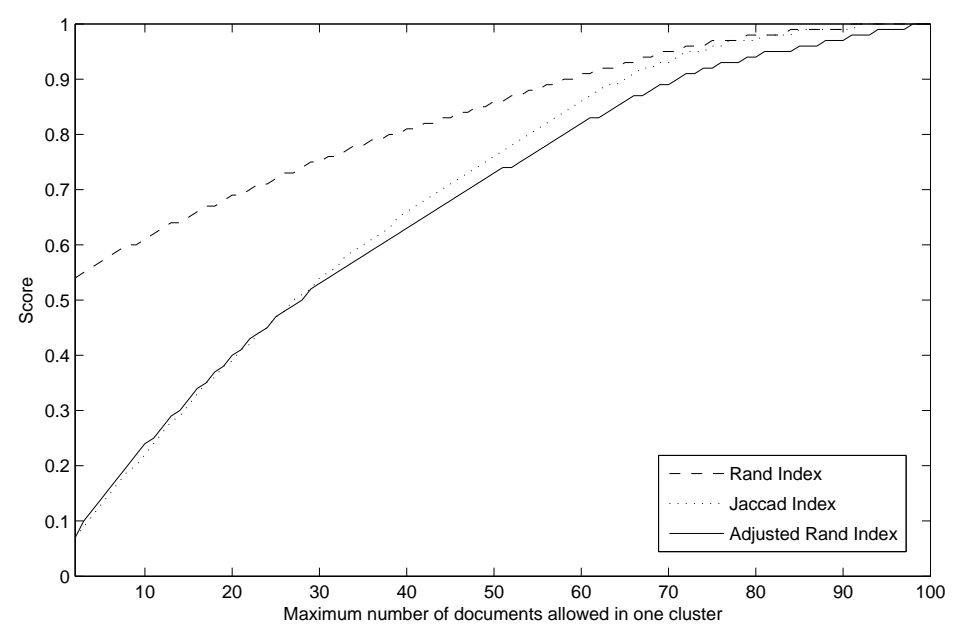

Figure 4.5: RI, ARI and JI Analysis

clusters of the gold standard cluster jaguar car.

QSC was compared with other algorithms using index based evaluation measures (RI, ARI and JI) however, these measures have many issues $[113,94]$. One of the problems is that they do not handle fine-grained clustering solutions. For example, if a gold standard $G$ has a cluster $g_{i}$ that contains 90 documents and the clustering solution $C$ has further refined the cluster $g_{i}$ into two distinct clusters $c_{j}$ and $c_{j+1}$, then the index based evaluation measure (RI, ARI and JI) will heavily penalize the clustering solution $C$. Although, the fine-grain clustering solution $C$ is consistent with the coarser solution $G$. In fact, the clustering solution $C$ may even be a better solution because it provides the distinctiveness which is not provided by the gold standard. Hence, using the index based evaluation measures (RI, ARI and JI) are not suitable to use when the data set can have fine grained clustering solutions.

It is worth mentioning that Recall, Precision, Entropy and F1-measure are biased towards pure clusters and trivial solutions such as all singleton clusters are not penalized. Therefore, we would need combination of index based evaluation measures (such as RI, ARI or JI) and purity based evaluation measure (such as F1-measure) to get a reliable evaluation of a 
clustering method.

Figure 4.5 shows the phenomena of heavy penalty of ARI and JI as compared to Rand Index (RI) [70] on sub clusters. This experiment was performed on the AMBIENT and MORESQUE datasets by evaluating the perfect sub-clusters that gradually increased the limit of the maximum number of documents allowed in a cluster from 2 to 98 . All the documents were perfectly assigned to the clusters and the values of RI, ARI and JI were computed at each iteration. The lowest value of RI, ARI and JI were $0.54,0.05$ and 0.04 respectively when the maximum allowed number of documents in sub clusters were 2. Figure 4.5 shows ARI and JI penalize small clusters and small sub-clusters heavily. The gold standard in our dataset had very unbalanced number of clusters. A few clusters were very small, and had 2 documents in a cluster and others were very large and had more than 90 documents. It was observed that the comparison based on ARI and JI is suitable only when the gold standard does not have sub clusters and all the clusters have almost the same number of members.

\subsection{Summary}

This chapter presents a new description-centric search result clustering algorithm QSC that exploits query senses to generate meaningful cluster labels and use syntactic, and semantic features of documents to generate quality clusters.

The results section in this chapter shows that QSC outperforms existing algorithms. QSC is computationally faster and provides better quality clusters with meaningful labels as compared to other algorithms, hence it has the potential to be applied to real time search result clustering applications. 


\section{Chapter 5}

\section{Soft Subspace Clustering}

\subsection{Introduction}

Clustering methods try to find similar documents and group them together in different clusters. Documents are generally represented in a Vector Space Model, where each distinct term is treated as a feature. Hence, the feature space becomes very large. In order to cluster in a large feature space, usually a dimensionality reduction techniques are applied to reduce the number of features before clustering the data. However, Kriegel et al. [83] outlined the limitations of using dimensionality reduction techniques and encourage researchers to develop subspace clustering methods.

Subspace clustering is categorized into soft and hard subspace clustering methods. Generally, soft subspace clustering methods are more suitable for clustering a text dataset because of their ability to partially represent a feature of the document in a subspace.

Common soft subspace clustering methods for text data, focus on finding different clusters in subspaces using a weighted distance measure. The weighting scheme heavily affects the clustering method and requires special consideration. Since text data has a semantic information along with a syntactic information, a weighting scheme, which uses a semantic information, is more likely to generate a better clustering solution. 


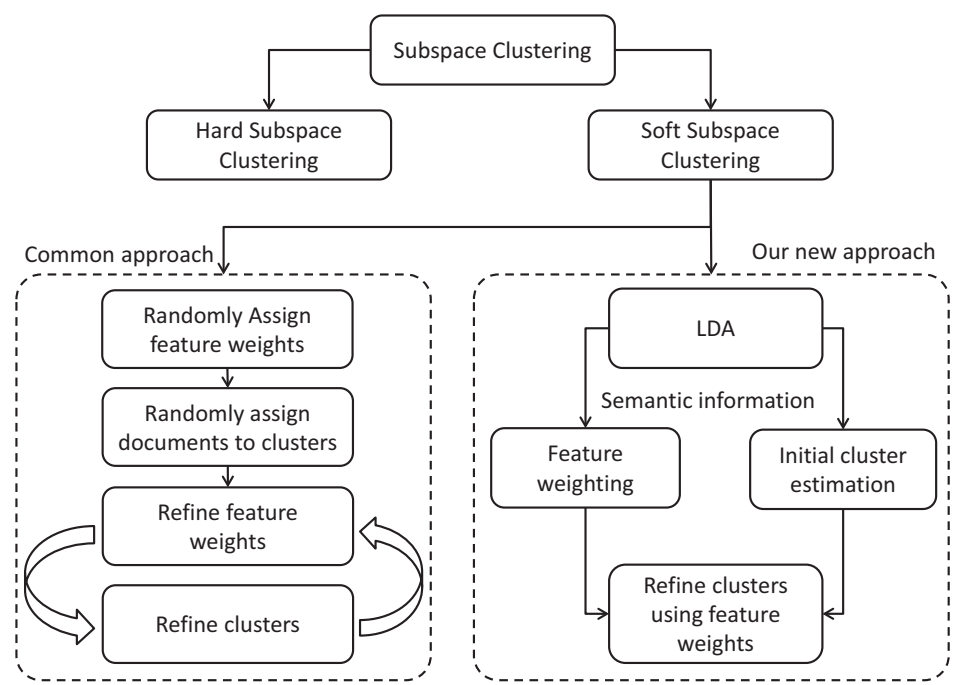

Figure 5.1: Differences between subspace clustering approaches and our new approach

The most popular soft subspace methods include FWKM [78], EWKM [77] and FGKM [26]. The main issue with these methods is that they ignore the semantic information of the documents, which might be helpful in improving the clustering process.

Latent Dirichlet Allocation (LDA) is a popular topic modeling method which can be used to extract semantic information from a collection of documents. LDA is based on a generative model, where a document is assumed to be generated from the distribution of terms which form a special theme or topic. We treat topics (generated from the LDA model) as subspaces because each topic specifies a soft subset of related terms (features). Subspaces generated by the LDA can be utilized in initializing the clusters.

This chapter introduces a novel soft subspace clustering method, Dirichlet weighted K-mean (DWKM), which uses a probabilistic model to extract semantic information from documents for weighting features. DWKM creates a feature weight matrix from the probability distribution of terms in 
subspaces and develops a weighted distance measure for finding similar documents in relevant subspaces.

DWKM uses LDA model to compute the probabilities that a term is relevant in a subspace (topic/subset of terms). These probabilities can represent the semantic information and are used as term or feature weightings in our soft subspace clustering to improve the clustering process. Figure 5.1 shows the differences between existing subspace clustering approaches and our new approach. Common existing soft subspace clustering methods use a random approach to initialize weightings and randomly assign objects to clusters. The feature weightings and clusters are refined iteratively. DWKM first uses LDA to assign the feature weights and assign objects to the initial clusters. Then DWKM iteratively refines the clusters according to the feature weights.

This chapter is organized as follows: section 5.2 discusses the related work; section 5.3 describes our proposed method; section 5.4 explains the experimental design and section 5.5 presents results along with discussions and section 5.6 provides a summary of the chapter.

\subsection{Existing Soft Subspace Clustering Algortihms}

Recent approaches such as FWKM [78], EWKM [77] and FGKM [26, 25] use k-means type variable weighting algorithms and formulate a minimization problem for data clustering. FWKM uses Lagrange multiplier and forms a polynomial weighting formula to compute the feature weights and iteratively refines the clusters using the following objective function.

$$
\sum_{i=1}^{k} \sum_{j=1}^{n} u_{i j} \sum_{t=1}^{m} \lambda_{i t}\left[\left(\mu_{i t}-d_{j t}\right)^{2}+\sigma\right]
$$

where

- $u$ is a $k \times n$ binary matrix representing the assignment of objects to clusters. $u_{i j}=1$ iff object $j$ is in cluster $i, u_{i j}=0$ otherwise. 
- $\lambda$ is $k \times m$ feature weight matrix. It represents $k$ subspaces in rows and $m$ features in columns. The value in a cell is a weight of the feature to its corresponding subspace and the value ranges from 01. The sum of the weights of all features in a subspace is 1. i.e. $\sum_{t=1}^{m} \lambda_{i t}=1,1 \leq i \leq k, 0<\lambda_{i t}<1$

- $\mu$ is a $k \times m$ matrix representing the mean value of a feature in a cluster.

- $d_{j t}$ represents a feature $t$ of the $j^{t h}$ object $^{1}$.

- $\sigma$ is an average spread/variance of all the features in a dataset.

EWKM clusters the data in a similar fashion but uses the exponential weighting formula to compute the feature weights. Its objective function is similar to equation 5.1, but instead of using $\sigma$, it uses Shannon entropy to control the weights. FGKM has a slightly different approach, it not only uses the individual feature weightings but also uses the feature group weightings scheme. The feature groups weightings are computed by combining features into different groups and then assigning weights to those groups.

The above soft subspace clustering methods ignore the semantic information of the documents in a clustering process. The main motivation of our research work is to investigate the use of semantic information (e.g. topics) of documents in soft subspace clustering process.

\subsubsection{Latent Dirichlet Allocation}

Latent Dirichlet Allocation (LDA) [15] extracts topics/themes from documents, which have semantic information. It is widely used in other domains such as topic modeling [16] and Entity Resolution [12]. The topics

\footnotetext{
${ }^{1}$ for clustering a collection of documents, $d_{j t}$ is often the term-frequency of a term in a document
} 


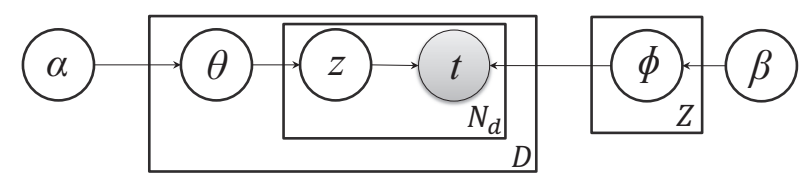

Figure 5.2: A common LDA graphical model using plate notation.

generated by LDA can be considered as subspaces and for each subspace, LDA facilitates to compute a term weight. Our soft subspace clustering method is related to FWKM and EWKM, however our method uses LDA based weighting scheme to utilize the semantic information of the documents.

LDA is a probabilistic model with an assumption that a document is a random mixture over latent topics and each topic is a distribution over terms. The two main parameters in this model are topic-document distributions $\theta$ and topic-term distributions $\phi$.

Figure 5.2 represents a graphical model for LDA. Arrows represent conditional dependencies between two variables and plates/rectangles represent loop or repetition of the variable mentioned in the corner of the plate. The shaded circle represents the observed variable while unshaded represent unobserved variables. Hyperparameter $\alpha$ is a prior on topic distribution. High value of $\alpha$ favors topic distributions with more topics and low value $(<1)$ of $\alpha$ favors topic distribution with a few topics. Hyperparameter $\beta$ is a prior on term distribution in every topic, which controls the number of times terms are sampled from a topic. The LDA model infers three latent variables $\theta, \phi$ and $z$ (topics) while observing $t$ (terms) in a document set $D$.

In Figure 5.2, the inner plate $(z$ and $t$ ) denotes the continuous sampling of topics and terms until $N_{d}$ terms are created from document $d$. The outer plate (which is surrounding $\theta$ ) denotes the continuous sampling of a topic distribution for each document $d$ in a document set $D$. The plate surrounding $\phi$ denotes the continuous sampling of a term distribution over each topic $z$ until a total of $Z$ topics are generated. More details of LDA 
can be found in [16].

To the best of our knowledge, our method DWKM is the first attempt that applies LDA to assign weights and use it in text soft subspace clustering.

\subsection{Our LDA Weighted K-Means Model}

This section presents our new subspace clustering method which builds on LDA for document clustering ${ }^{2}$. Figure 5.3 shows the overall design of our method. The documents are pre-processed by implementing stop words filtration, low frequency words filtration and WordNet lemmatization. Then we use LDA based on Gibbs sampling to generate two matrices: topic-document matrix $\theta$ and topic-term matrix $\phi . \theta$ is then used for initializing the clusters and $\phi$ is used as feature weights for refining the clusters.

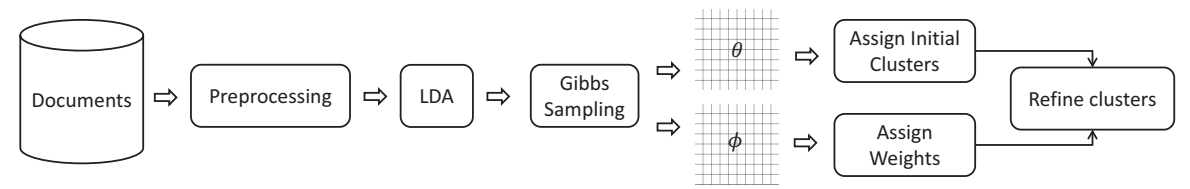

Figure 5.3: System diagram of our new method. $\theta$ and $\phi$ are the topicdocument and topic-term matrices respectively.

\subsubsection{Gibbs Sampling}

We implemented LDA model in an unsupervised way (without training datasets) using Gibbs sampling algorithm explained in [118]. The Gibbs sampling algorithm iteratively computes the conditional probability of assigning an occurrence of a term (token of a term) to each topic. The common Gibbs sampling method provides the estimates of the posterior distribution over $z$ (topics) but does not provides $\theta$ and $\phi$. However, we can

\footnotetext{
${ }^{2}$ The code of our method was implemented using lingpipe toolkit (http://aliasi.com/lingpipe/)
} 
use the Gibbs sampling method to approximate $\theta$ and $\phi$ from posterior estimates of $z$.

For each token $i$ (an occurrence of a term), let $v_{i}, d_{i}, z_{i}$ denote the term for the token, the document for the token and the topic of the token respectively in a document collection. The Gibbs sampling method iteratively processes each term token in the document collection and estimates the conditional probability of assigning the current term token to an individual topic, based on the topic assignments to all other term tokens. The conditional distribution is formalized as:

$$
\operatorname{Prb}\left(z_{i}=r \mid \mathbf{z}_{-i}, \ldots\right)
$$

where $z_{i}=r$ is the assignment of $i^{\text {th }}$ token to topic $r . \mathbf{z}_{-i}$ denotes the topic assignment of all the tokens excluding the $i^{\text {th }}$ token. Other variables for equation 5.2 represented by (...) are $v_{i}, d_{i}, \mathbf{v}_{-i}, \mathbf{d}_{-i}, \alpha$ and $\beta . \mathbf{v}_{-i}$ represents all terms tokens except the $i^{\text {th }}$ term token and $\mathbf{d}_{-i}$ represents document tokens except the $i^{t h}$ document token. Griffiths and Steyvers [118] provided a simple way to compute Equation 5.2 as:

$$
\operatorname{Prb}\left(z_{i}=r \mid \mathbf{z}_{-i}, \ldots\right) \propto \frac{\mathcal{C}_{r v_{i}}^{(1)}+\beta}{\sum_{l=1}^{m} \mathcal{C}_{r l}^{(1)}+m \beta} \frac{\mathcal{C}_{r d_{i}}^{(2)}+\alpha}{\sum_{z=1}^{Z} \mathcal{C}_{z d_{i}}^{(2)}+Z \alpha}
$$

where $\mathcal{C}^{(1)}$ and $\mathcal{C}^{(2)}$ are $Z \times m$ and $Z \times D$ matrices respectively and $Z, m$, $D$ are the number of topics, terms and documents respectively. The cell values of these matrices represent the frequency of the term/document for the corresponding topics. $\mathcal{C}_{r v_{i}}^{(1)}$ denotes the number of times the term $v_{i}$ is assigned to the topic $r$ excluding the $i^{t h}$ instance and $\mathcal{C}_{r d_{i}}^{(2)}$ denotes the number of times a term token in document $d$ is assigned to the topic $r$ excluding the $i^{t h}$ instance. 


\subsubsection{Generating $\theta$ and $\phi$}

After applying the Gibbs sampling algorithm, we create two matrices: 1) $\phi$ topic-term matrix and 2) $\theta$ topic-document matrix. These matrices are generated from the two count matrices $\mathcal{C}^{(1)}$ and $\mathcal{C}^{(2)}$ as follows:

$$
\phi_{r t}=\frac{\mathcal{C}_{r t}^{(1)}+\beta}{\sum_{l=1}^{m} \mathcal{C}_{r l}^{(1)}+m \beta}, \theta_{r j}=\frac{\mathcal{C}_{r j}^{(2)}+\alpha}{\sum_{z=1}^{Z} \mathcal{C}_{z j}^{(2)}+Z \alpha}
$$

$\phi$ corresponds to the probability that a term $t$ is assigned to topic $r$ and $\theta$ corresponds to the probability that a document $j$ is assigned to topic $r$.

The rows of the topic-document matrix $\theta$ represent topics and the columns represent documents. The cells of $\theta$ represent the probability that a document has the corresponding topic. We use this matrix to form the initial clusters. One should note that LDA naturally provides a simple way for clustering the documents. However, this clustering is not soft subspace clustering. The following is a way to improve the clusters generated from LDA by utilizing the information from LDA and forming a soft subspace clustering method.

In LDA model, each term is a feature and each topic corresponds to a subspace, therefore topic-term matrix $\phi$ can be considered as a feature weight matrix for different subspaces where each feature or term has a degree of presence in all subspaces or topics. We used the values of topicterm matrix $\phi$ for determining relevant subspaces and developed a new weighted distance measure, which finds similar documents in relevant subspaces.

\subsubsection{Objective Function}

We perform clustering by formulating the clustering as a minimization problem and our objective is to minimize the sum of squared distances between documents and the nearest cluster centers weighted by different subspaces. The objective function is similar to the objective functions 
(equation 5.5) of the FWKM or EWKM, however, we do not include $\sigma$ or Shannon entropy because we are already controlling the feature weighting using two hyper parameters of LDA model ( $\alpha$ and $\beta$ ). Moreover, the objective function of DWKM uses previously computed LDA based feature weights instead of computing the feature weights in iterative manner.

Let $D=\left\{d_{1}, d_{2}, d_{3}, \ldots, d_{n}\right\}$ be a set of $n$ documents and $T=\left\{t_{1}, t_{2}, t_{3}, \ldots, t_{m}\right\}$ represents $m$ terms in the documents. Then the objective function for clustering the $n$ documents into $k$ clusters can be defined as:

$$
\sum_{i=1}^{k}\left(\sum_{j=1}^{n} \sum_{t=1}^{m} \delta_{i j} \phi_{i t}\left(\mu_{i t}-d_{j t}\right)^{2}\right)
$$

where

- $\delta$ is a $k \times n$ binary matrix representing the assignment of documents to clusters. $\delta_{i j}=1$ iff document $j$ is in cluster $i, \delta_{i j}=0$ otherwise.

- $\phi$ is $k \times m$ topic-term matrix generated from LDA model. It represents $k$ subspaces in rows and $m$ terms in columns. The value in a cell is a weight of the term to its corresponding subspace and the value ranges from 0-1. The sum of the weights of all terms in a subspace is 1. i.e. $\sum_{t=1}^{m} \phi_{i t}=1,1 \leq i \leq k, 0<\phi_{i t}<1$

- $\mu$ is a $k \times m$ matrix representing the mean value of a term in a cluster. It is calculated as:

$$
\mu_{i t}=\frac{\sum_{j=1}^{n} \delta_{i j} d_{j t}}{\sum_{j=1}^{n} \delta_{i j}}
$$

- $d_{j t}$ represents a term $t$ (a feature) of the $j^{\text {th }}$ document, which is the term-frequency of the term in the document.

We iteratively assign documents to their nearest cluster centers until the algorithm converges. We minimize the objective function by updating $\delta$ using the following: 


$$
\delta=\left\{\begin{array}{l}
\delta_{i j}=1, \text { if } i=\operatorname{argmin}_{x} \operatorname{dist}\left(\mu_{x}, d_{j}\right) \\
\delta_{i j}=0, \text { otherwise }
\end{array}\right.
$$

where $\operatorname{dist}\left(\mu_{x}, d_{j}\right)$ is defined as

$$
\operatorname{dist}\left(\mu_{x}, d_{j}\right)=\sum_{t=1}^{m} \phi_{x t}\left(\mu_{x t}-d_{j t}\right)^{2}
$$

Equation 5.8 defines our distance measure. Inspired from k-means, our distance measure computes the distance of a document from the cluster centers by using a LDA parameter $\phi$, which provides a semantic based feature weighting to different subspaces. Higher value of the probability that a term is assigned to a topic, indicates that the term has a higher degree of presence in a subspace. Therefore the difference between a term in the document and the mean value of the term in the cluster for that particular term is more important. The use of LDA differentiates our method from other soft subspace clustering methods.

\subsubsection{Our Algorithm: DWKM}

Our Dirichlet Weighted K-mean algorithm is a modified version of k-means algorithm. The details are shown in Algorithm 5.1.

Algorithm 5.1 takes two arguments: a document set and the number of clusters and outputs the clustering solution. The algorithm performs preprocessing step on the documents, which includes stop word removal, lemmatization and tokenization of words. Then the algorithm randomly assigns all term tokens to $Z$ topics and performs Gibbs sampling. Once $\phi$ and $\theta$ matrices are generated. Line 4 of the algorithm groups documents to different clusters according to their highest probability using $\theta$. The algorithm then, fine tunes the clusters by repeating the update and assignment steps according to equation 5.6 and 5.7 until convergence criteria is met. The convergence criteria terminates the loop if there is no more docu- 


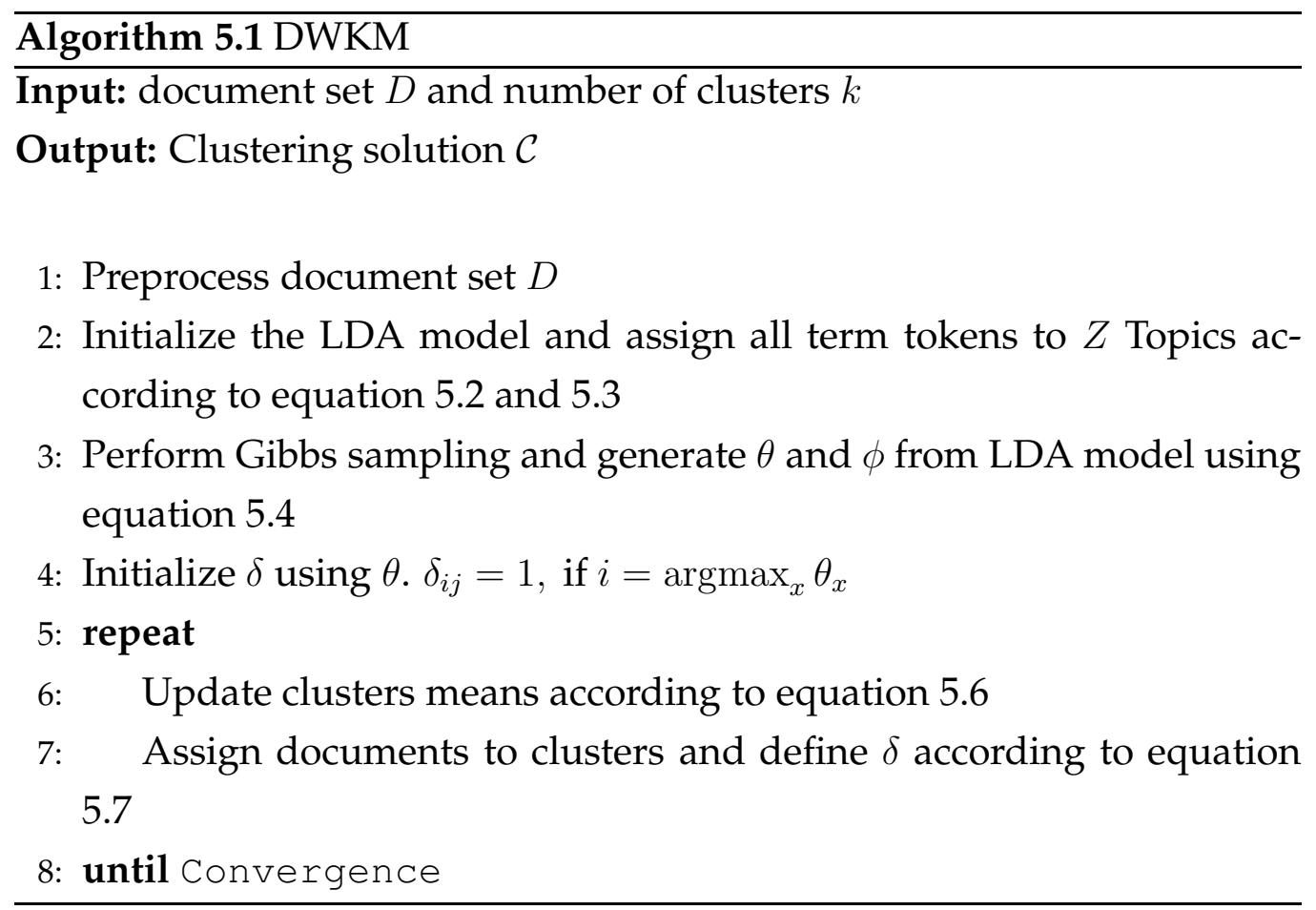

ments to relocate to any clusters or the total number of specified iterations exceeds the predefined limit.

\subsection{Experimental Setup}

Our experiments are designed based on two recent papers [26] and [77]. Our method DWKM was evaluated on four synthetic and six real world datasets, and compared with five clustering methods using different cluster quality measures. Our experiments are based on the assumption that we already know the number of clusters (i.e. The value of $k$ ) for all clustering methods. 


\subsubsection{Datasets}

The synthetic datasets SD1, SD2, SD3, SD4 were generated according to [26]. Each consists of 6000 objects, 200 features, three subspaces and three clusters. The noise level in SD1, SD2, SD3 and SD4 are 0, 0.2, 0 and 0.2 respectively (as described in [26]). The percentage of missing values in DS1, DS2, DS3 and DS4 are 0, 0, 0.12, 0.12 respectively. Detailed information about how to reproduce the synthetic datasets can be found in [26].

The six real-word datasets with two or more clusters from 20-Newsgroup ${ }^{3}$ are the same as [77]. Table 5.1 shows the details of these six datasets. The dataset D1, D2 and D3 are easier than datasets D4, D5 and D6. D1 and D2 have semantically different clusters whereas D4 and D5 have semantically related clusters. D3 and D6 have unbalanced clusters (as shown in table 5.1).

\subsubsection{Evaluation Measures}

In order to compare our method with other methods, we used two evaluation measures: Cluster Accuracy [105] and F-measure [30] for synthetic dataset and three evaluation measures: F-measure, Normal Mutual Information(NMI) [154] and Entropy [153] for the real-world datasets. These measures are chosen based on [77] and [26]. Lower entropy values of a clustering solution indicates the clustering solution has better quality, whereas higher values of all other evaluation measures indicate better cluster quality. These evaluation measures were previously described in chapter 3.

\subsection{Results}

We compared our method DWKM with k-means, LDA based simple clustering, FWKM [78], EWKM [77] and FGKM [26].

\footnotetext{
${ }^{3}$ http://qwone.com/ jason/20Newsgroups/
} 
Table 5.1: Six real world datasets created from 20-Newsgroup dataset

\begin{tabular}{clc}
\hline dataset & clusters & \# of docs \\
\hline D1 & alt.atheism & 100 \\
& comp.graphics & 100 \\
D2 & comp.graphics & 100 \\
& rec.sport.baseball & 100 \\
& sci.space & 100 \\
& talk.politics.mideast & 100 \\
D3 & comp.graphics & 120 \\
& rec.sport.baseball & 100 \\
& sci.space & 59 \\
& talk.politics.mideast & 20 \\
D4 & talk.politics.mideast & 100 \\
& talk.politics.misc & 100 \\
D5 & comp.graphics & 100 \\
& comp.os.ms-windows & 100 \\
& rec.autos & 100 \\
& sci.electronics & 100 \\
D6 & comp.graphics & 120 \\
& comp.os.ms-windows & 100 \\
& rec.autos & 59 \\
& sci.electronics & 20 \\
\hline
\end{tabular}




\subsubsection{Comparison}

K-means and LDA based simple clustering algorithm were implemented in lingpipe toolkit (http://alias-i.com/lingpipe/)). We provided predefined number of clusters as a parameter for both algorithms. The simple LDA clustering algorithm uses the same initial steps described in our method without the cluster refinement step. We treated initial clusters as final clusters and skipped the loop which refines the cluster using feature weights. The parameters for LDA are number of topics $=$ number of clusters in ground truth, number of clusters = number of clusters in ground truth, $\alpha=0.1$ and $\beta=0.01$. We tuned the parameter $\alpha$ and $\beta$ for the best performance. FWKM, EWKM and FGKM clustering algorithm were implemented in Weka ${ }^{4}$ and we used standard parameters as described by the authors.

Table 5.2: Comparison of clustering methods on synthetic dataset using Accuracy (AC) and F-measure (FM). The values on left are the mean values of 100 runs and the values in parenthesis are standard deviation of 100 runs.

\begin{tabular}{|c|c|c|c|c|c|c|c|}
\hline datasets & Metric & k-means & LDA & FWKM & EWKM & FGKM & DWKM \\
\hline \multirow{2}{*}{ SD1 } & $\mathrm{AC}$ & $0.65(0.09)$ & $0.66(0.11)$ & $0.77(0.14)$ & $0.69(0.10)$ & $0.82(0.16)$ & $0.87(0.15)$ \\
\hline & $\mathrm{FM}$ & $0.63(0.13)$ & $0.65(0.09)$ & $0.73(0.19)$ & $0.59(0.13)$ & $0.75(0.22)$ & $0.81(0.20)$ \\
\hline \multirow{2}{*}{ SD2 } & AC & $0.63(0.04)$ & $0.68(0.06)$ & $0.76(0.10)$ & $0.72(0.13)$ & $0.87(0.16)$ & $0.92(0.15)$ \\
\hline & $\mathrm{FM}$ & $0.64(0.05)$ & $0.69(0.09)$ & $0.75(0.12)$ & $0.63(0.17)$ & $0.82(0.22)$ & $0.88(0.21)$ \\
\hline \multirow{2}{*}{ SD3 } & $\mathrm{AC}$ & $0.62(0.04)$ & $0.64(0.07)$ & $0.67(0.07)$ & $0.70(0.09)$ & $0.94(0.13)$ & $0.94(0.12)$ \\
\hline & FM & $0.62(0.06)$ & $0.63(0.13)$ & $0.64(0.11)$ & $0.59(0.11)$ & $0.91(0.18)$ & $0.92(0.17)$ \\
\hline \multirow{2}{*}{ SD4 } & $\mathrm{AC}$ & $0.60(0.04)$ & $0.61(0.15)$ & $0.61(0.06)$ & $0.69(0.08)$ & $0.91(0.13)$ & $0.93(0.13)$ \\
\hline & FM & $0.59(0.05)$ & $0.60(0.16)$ & $0.60(0.07)$ & $0.58(0.11)$ & $0.88(0.18)$ & $0.90(0.19)$ \\
\hline
\end{tabular}

The performance of all six clustering algorithms for synthetic dataset is shown in Table 5.2 and for real-world dataset is shown in Table 5.3.

Table 5.2 shows the comparison of clustering methods in terms of Ac-

\footnotetext{
${ }^{4}$ The code for FWKM, EWKM and FGKM was provided by the authors
} 
Table 5.3: Comparison of clustering methods in terms of F-measure, NMI and Entropy on six real-world datasets created from 20-Newsgroup dataset. The values listed in the table are the mean values of 100 runs of five clustering methods on six real-world datasets

\begin{tabular}{clcccccc}
\hline Datasets & Metric & k-means & LDA & FWKM & EWKM & FGKM & DWKM \\
\hline \multirow{2}{*}{ D1 } & F-measure & $\mathbf{0 . 9 6}$ & $\mathbf{0 . 9 6}$ & 0.95 & $\mathbf{0 . 9 6}$ & $\mathbf{0 . 9 6}$ & $\mathbf{0 . 9 6}$ \\
& NMI & 0.78 & 0.78 & 0.79 & 0.83 & 0.85 & $\mathbf{0 . 8 6}$ \\
& Entropy & 0.21 & 0.21 & 0.20 & 0.16 & 0.15 & $\mathbf{0 . 1 3}$ \\
D2 & F-measure & 0.93 & 0.92 & 0.90 & 0.91 & 0.94 & $\mathbf{0 . 9 6}$ \\
& NMI & 0.80 & 0.78 & 0.75 & 0.76 & 0.78 & $\mathbf{0 . 8 0}$ \\
& Entropy & 0.19 & 0.24 & 0.25 & 0.23 & 0.17 & $\mathbf{0 . 1 5}$ \\
D3 & F-measure & 0.89 & 0.90 & 0.95 & 0.95 & 0.95 & $\mathbf{0 . 9 6}$ \\
& NMI & 0.71 & 0.72 & 0.84 & 0.86 & 0.87 & $\mathbf{0 . 8 8}$ \\
& Entropy & 0.28 & 0.20 & 0.15 & 0.11 & 0.10 & $\mathbf{0 . 0 8}$ \\
D4 & F-measure & 0.88 & 0.90 & 0.90 & 0.94 & 0.95 & $\mathbf{0 . 9 6}$ \\
& NMI & 0.47 & 0.55 & 0.60 & 0.72 & 0.75 & $\mathbf{0 . 7 8}$ \\
& Entropy & 0.52 & 0.30 & 0.40 & 0.28 & 0.27 & $\mathbf{0 . 2 0}$ \\
D5 & F-measure & 0.70 & 0.75 & 0.86 & 0.89 & 0.90 & $\mathbf{0 . 9 2}$ \\
& NMI & 0.38 & 0.48 & 0.64 & 0.68 & 0.70 & $\mathbf{0 . 7 3}$ \\
& Entropy & 0.61 & 0.41 & 0.35 & 0.31 & 0.30 & $\mathbf{0 . 2 9}$ \\
D6 & F-measure & 0.65 & 0.81 & 0.92 & 0.92 & 0.93 & $\mathbf{0 . 9 4}$ \\
& NMI & 0.37 & 0.68 & 0.73 & 0.75 & 0.76 & $\mathbf{0 . 7 8}$ \\
& Entropy & 0.53 & 0.28 & 0.23 & 0.23 & 0.22 & $\mathbf{0 . 1 9}$ \\
\hline \multirow{2}{*}{ N-.9. } & & & & & &
\end{tabular}


curacy and F-measure on four synthetic datasets. The values in bold represent the best results. In general, DWKM performs better than other clustering methods in terms of both Accuracy and F-measure on the synthetic datasets. The Accuracy and F-measure values on datasets SD1 and SD2 for DWKM and FGKM have large gaps, whereas the differences of the values on datasets SD3 and SD4 are relatively smaller. The LDA based simple clustering performed better than standard k-means, but performed worse than soft subspace clustering algorithms.

Table 5.3 shows the mean values of F-measure, NMI and Entropy for kmeans, FWKM, FGKM and DWKM clustering methods on six real-world datasets. In general, on the six real-world data set DWKM performed better than other clustering methods in terms of F-measure, NMI and Entropy values. The D1 dataset is the easiest dataset. K-means, EWKM, FGKM and DWKM have the same F-measure value 0.96 on D1 dataset, which means these clustering methods produced equally good clustering solutions. However, if we consider the NMI and Entropy values along with F-measure values of the D1 dataset, we can see that DWKM performed slightly better than other clustering methods. The LDA based simple clustering followed the same trend as in synthetic datasets and performed better than standard k-means, but worse than soft subspace clustering algorithms.

Table 5.4: Percentage improvement of DWKM over FGKM in terms of Accuracy $(\mathrm{AC})$ and F-measure (FM) on synthetic datasets

\begin{tabular}{ccc}
\hline & AC \% (IMP) & FM \% (IMP) \\
\hline SD1 & 5.75 & 7.41 \\
SD2 & 5.43 & 6.82 \\
SD3 & 0.00 & 1.09 \\
SD4 & 2.15 & 2.22 \\
\hline
\end{tabular}

It was also observed from the results that DWKM performed well on 
Table 5.5: Percentage improvement of DWKM over FGKM in terms of Fmeasure (FM), NMI and Entropy (EN) on real datasets

\begin{tabular}{cccc}
\hline & FM \% (IMP) & NMI \% (IMP) & EN \% (IMP) \\
\hline D1 & 0.000 & 1.163 & 2.299 \\
D2 & 2.083 & 2.500 & 2.353 \\
D3 & 1.042 & 1.136 & 2.174 \\
D4 & 1.042 & 3.846 & 8.750 \\
D5 & 4.255 & 4.110 & 1.408 \\
D6 & 2.105 & 2.564 & 3.704 \\
\hline
\end{tabular}

data with different level of difficulties (data without noise, with noise, with balanced clusters and with unbalanced clusters). This shows that our semantic weighting of subspaces derived from LDA is reasonably effective for finding clusters in different types of data. Moreover the LDA based simple clustering algorithm performed much better than k-means algorithm when datasets had semantically related clusters (results of D4 and D5). It was also noted that the use of the cluster refinement step based on feature weighting of LDA model boosted the performance of clustering. The DWKM algorithm without the cluster refinement step, performed better than k-means algorithm and slightly worse than other clustering methods.

Table 5.4 and table 5.5 provide percentage improvement of DWKM over FGKM on synthetic datasets and real datasets respectively. The results in all tables suggest that DWKM is a better clustering method. We further investigate the performance of all clustering methods by conducting a statistical analysis. 
Table 5.6: P-values of unpaired ttest of DWKM and FGKM on synthetic datasets

\begin{tabular}{ccccc}
\hline & SD1 & SD2 & SD3 & SD4 \\
\hline Accuracy & $\mathbf{0 . 0 2 3 7}$ & $\mathbf{0 . 0 2 3 7}$ & $\mathbf{1}$ & 0.278 \\
F-measure & $\mathbf{0 . 0 4 4 9}$ & $\mathbf{0 . 0 4 4 9}$ & 0.6867 & 0.4457 \\
\hline
\end{tabular}

\subsubsection{Statistical Analysis}

We performed two types of statistical tests: 1) unpaired ttest and 2) paired Wilcoxon statistical significance test [138] by considering DWKM as the control group. The unpaired ttest was performed using the standard deviation and mean values of evaluation measures listed in table 5.2. In general the results from unpaired ttest showed that DWKM achieved statistically significant improvement over three methods k-means, FWKM and EWKM on all synthetic datasets with p-value less then $\mathbf{0 . 0 5}$. The p-values of unpaired ttest computed for FGKM on SD1 and SD2 synthetic datasets are less than $\mathbf{0 . 0 5}$, which indicates that our method DWKM has statistical significant improvement on SD1 and SD2 over FGKM. The performance of our method on other SD3 and SD4 synthetic dataset was found to be comparable to FGKM.

For the six real-world dataset we used paired Wilcoxon statistical significance test. The p-values of F-measure, NMI and Entropy values for FGKM were $\mathbf{0 . 0 3 0 5}, \mathbf{0 . 0 0 2 8}$ and $\mathbf{0 . 0 2 2 8}$ respectively. In general the $\mathrm{p}$-values for all five clustering methods were found to be less than $\mathbf{0 . 0 5}$, which suggested that our method DWKM shows a better performance and significant improvement over five clustering methods. 


\subsection{Summary}

In this chapter, we introduced a new soft subspace clustering method which uses LDA model to weight the features in the subspaces for clustering documents. The LDA model was implemented using a standard Gibbs sampling algorithm, and two matrices: topic-term and topic-documents were generated. We used the topic-term matrix to develop a new weighted distance measure, where topics are used as subspaces. We developed a kmean based soft subspace clustering method based on our new weighted distance measure. The algorithm is initialized using the topic-document matrix, where documents with the same topics from the initial clusters.

Our new method DWKM, was found to have a statistically significant improvement over recently developed soft subspace clustering methods on synthetic and real-world datasets. 


\section{Chapter 6}

\section{Multi-View Multi-Objective Evolutionary Approach}

\subsection{Introduction}

Thousands of clustering algorithms have been proposed in the last 50 years [75]. As no single clustering algorithm is suitable for all types of problems, researchers have been trying different techniques for combining different clustering algorithms, which are called clustering ensembles $[47,119,124,55,137,128]$.

The main goal of clustering ensembles is to solve the problem of producing a superior clustering solution from a given set of clustering solutions. This problem was previously approached by researchers from different angles and so far the best known approach for clustering ensembles is the median partition based approach [9] in which a single candidate clustering solution that has the maximum similarity from all candidate clustering solutions is selected as the final clustering solution.

As stated in the introduction chapter, clustering ensembles methods try to address two key issues: how to generate different candidate solutions and how to combine them in two steps. In step 1, a general practice is to use different clustering algorithms with different initialization parameters 
to generate different candidate clustering solutions. This common step 1 strategy might work for single view data sets. However, text data is generally multi-view and requires step 1 to generate a diverse set of candidate clustering solutions from different views.

As previously mentioned that a document can be represented by multiple views e.g., semantic view (i.e. topics, title, hypertext etc.) and syntactic view (i.e. term frequencies), and considering multiple views at the same time has already proven to result in better clusters [13]. Therefore, it is reasonable to use multiple views for generating different candidates in a clustering ensemble method.

In step 2, clustering ensembles methods generally choose the best clustering solution among a given set of clustering solutions. However, this might not lead to a better solution because each clustering solution generally consists of a mixture of high and low quality clusters ${ }^{1}$. In order to generate a better clustering solution, a selection of high quality clusters from different candidate clustering solutions should be combined instead of selecting one solution from the set of candidate clustering solutions.

Generally, clustering ensemble methods focus on optimizing a single objective function (i.e., either maximizing the inter-cluster distances or maximizing the intra-cluster similarity). However, in recent years the trend has shifted toward formulating clustering ensembles as multi-objective optimization problems to gain better results [89, 119, 62].

This chapter presents a Multi-view Multi-objective Evolutionary Algorithm (MMOEA) which uses a multi-objective optimization approach for improving document clustering, focusing on the following key ideas:

1. Generate different candidate clustering solutions for step 1 from multiple views of data.

2. Select the best combination of clusters from all candidate clustering

\footnotetext{
${ }^{1}$ high quality clusters are the ones that have high intra-cluster similarity and are dissimilar from other clusters
} 
solutions to form a new clustering solution instead of selecting an existing single clustering solution from the candidate clustering solutions.

- Using a multi-objective optimization approach instead of a single objective approach.

- Allowing overlapping clusters so that one document can be in multiple clusters. This is often desirable for document clustering because many documents can be classified under multiple topics or categories.

The chapter is organized as follows: section 6.2 discusses related methods for our approach; section 6.3 provides the details of the approach; section 6.4 describes the experimental setup; section 6.5 discusses the comparison of the approach with other methods and provides a statistical analysis; lastly section 6.6 provides the summary of the chapter.

\subsection{Existing Multi-Objective Clustering Methods}

Handl and Knowles [62,63] proposed a state-of-the-art evolutionary approach for multi-objective clustering named Multi-objective clustering algorithm with K-determination (Mock) based on Pareto Envelop based Selection Algorithm (PESA-II) [29]. They used a fitness function based on two objectives: connectedness and compactness of clusters. The placement of neighboring objects in the same cluster is called connectedness and is defined as:

$$
\operatorname{Conn}(C)=\sum_{i=1}^{N} \sum_{j=1}^{M} y_{i, x_{i j}}
$$

where $x_{i j}$ is the $j^{\text {th }}$ nearest neighbour of the $i^{t h}$ object $o_{i}$ and $M$ is the total number of neighbors that contribute to the measure and $y_{i, x_{i j}}$ is given by 


$$
y_{i, x_{i j}}=\left\{\begin{array}{ll}
\frac{1}{j} & \text { if } \nexists C_{k}: o_{i} \in C_{k} \wedge x_{i j} \in C_{k} \\
0, & \text { otherwise }
\end{array}\right\}
$$

The cluster compactness is how similar the objects are in a cluster of a clustering solution. It improves when the number of clusters are high, whereas improving connectivity requires less number of clusters. These conflicting objectives make the algorithm to explore interesting areas of the search space.

Another multi-objective clustering algorithm was proposed in [81]. It uses two objectives: the number of clusters and the intra-cluster variation (which is computed over all clusters). Both objectives are required to be minimized, although they are in conflict with each other. The algorithm uses Pareto dominance to discover a set of nondominated clustering solutions that are different from each other (diversity is preserved) by finding the smallest intra-cluster variance for the minimum number of clusters.

Bandyopadhyay et al. [8] introduced a multi-objective evolutionary algorithm that also performs fuzzy clustering. It uses two objective functions: $J_{m}$ criterion [88] and the Xie-Beni index [142], which is a distance between two closest clusters.

Other variations of multi-objective clustering algorithms include [42, 99, 112]. Mostly these algorithms used two objectives and their main focus was on intra-cluster distance that needed to be minimized. The main limitations of these algorithms were considering only single view of the data and selecting the best existing solution from the set of candidate solutions.

\subsection{Our Multi-View Multi-Objective Evolution- ary Approach}

Our method MMOEA, is based on multi-view clustering in which different clustering solutions are derived from different views of the data, and 
uses a modified version of NSGA-II [36] approach.

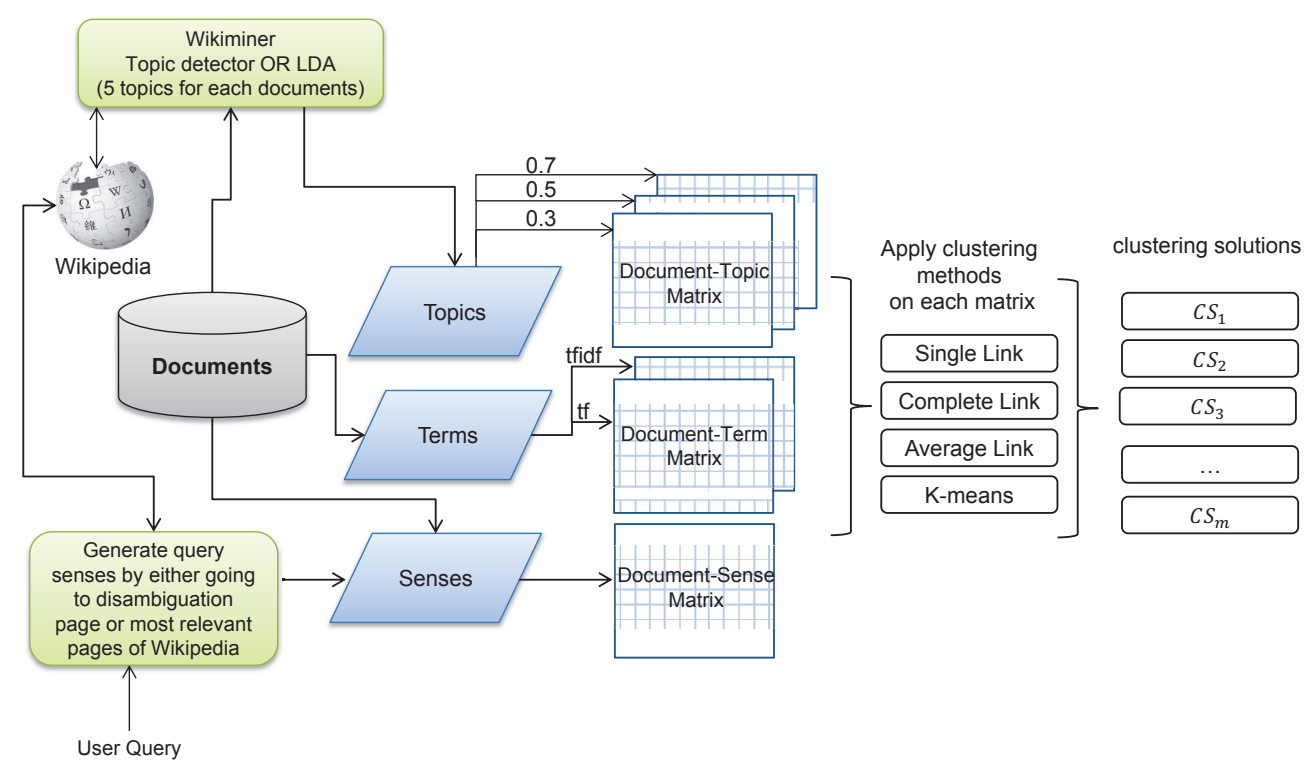

Figure 6.1: Process of generating 24 different clustering solutions.

\subsubsection{Initialization Method}

Unlike previous clustering ensemble methods, we use different views for generating initial candidate clustering solutions. In our method, we considered terms in documents (bag of words), user query senses and topics in documents as three views. We assume the set of documents was generated using a query to a search engine.

The term view is generated by extracting the terms in the documents. The query senses view is generated by parsing Wikipedia disambiguation pages and extracting different senses of that query ${ }^{2}$. The topics for each documents are generated using the topic detection component of Wikiminer toolkit ${ }^{3}$ [96] and Latent Dirichlet Allocation (LDA). Wikiminer

\footnotetext{
${ }^{2}$ We can skip the query sense view for datasets that do not provide queries

${ }^{3}$ for more information visit http:/ / wikipedia-miner.cms.waikato.ac.nz/
} 
generates the topics of a document by matching its terms with the titles of Wikipedia articles. Wikiminer is not able to generate multiple topics for documents that do not have sufficient terms corresponding to any Wikipedia article. Therefore, we use a simple implementation of LDA to tackle these (rare) cases and ensure that we will get at least five topics per document. The term view provides a syntactic representation of the document whereas the query sense and the topic view provide a semantic representation of the document.

We created two document-term matrices from the term view, one documentsense matrix from the query sense view and three document-topic matrices from the topic view of the documents.

In a document-term matrix, each row represents a document, each column represents a term, and a cell contains the weighted value of a term for a document. The first matrix is created using tfidf (a common weighting scheme) and the cell contains the tfidf score. The second matrix is created by simply computing the term frequencies (tf) for each cell of the matrix.

In a document-sense matrix, rows represent documents and columns represent different senses of the query. A cell contains 1 if the sense was present in the document and 0 if the sense is not present (exact string matching).

In a document-topic matrix, rows represent documents and columns represent topics. A cell contains 1 if the similarity score between the topic and any topics of the document is above a threshold, otherwise the value is 0 . We use $\mathrm{Wu}$ and Palmer similarity measure [141] to match topics and created three document-topic matrices based on three thresholds $(0.3,0.5$ and 0.7).

We then applied four different clustering algorithms: single link hierarchical clustering, complete link hierarchical clustering, average link hierarchical clustering and k-means clustering on the six matrices(described earlier) to generate 24 candidate clustering solutions.

Figure 6.1 depicts the initialization process. The total of 24 clustering 
solutions are generated from six matrices by applying four different clustering methods. The six matrices were generated from three views of the document.

\subsubsection{Genetic Representation}

Using MOEA for a clustering problem requires a representation of the clustering solutions, objective functions and genetic operators (crossover and mutation methods).

The representation scheme used in our method is a matrix based binary encoding scheme [68]. In this scheme each clustering solution or individual is represented in the form of a $k \times N$ matrix. In this matrix, columns represent documents and rows represent clusters. Figure 6.2 depicts a sample clustering solution and its matrix based encoding is shown in Figure 6.3. The value 1 in cell $(c, d)$ of the matrix means that the cluster $c$ includes the document $d$.

The key advantage of this representation is that it can represent overlapping clusters. Note that this representation would allow a clustering in which some documents are not allocated to any clusters.

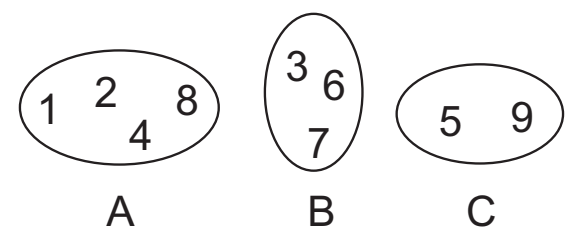

Figure 6.2: Sample Clustering

\subsubsection{Objective Functions}

Since a good clustering solution should have compact clusters and all clusters should be distinct from each other, therefore, we used intra-cluster similarity and inter-cluster distances to formulate our objective function. 


\begin{tabular}{|c|c|c|c|c|c|c|c|c|c|}
\hline & 1 & 2 & 3 & 4 & 5 & 6 & 7 & 8 & 9 \\
\hline A & 1 & 1 & 0 & 1 & 0 & 0 & 0 & 1 & 0 \\
\hline B & 0 & 0 & 1 & 0 & 0 & 1 & 1 & 0 & 0 \\
\hline C & 0 & 0 & 0 & 0 & 1 & 0 & 0 & 0 & 1 \\
\hline
\end{tabular}

Figure 6.3: A sample individual in a form of matrix-based binary encoding where columns represent the document ids and rows represent the cluster numbers

The only issue with these two criteria was that they were biased towards producing small (possibly similar) number of clusters, therefore we formulated a third objective function which is based on the number of clusters criterion, to get a good trade off between all three objective functions.

We implemented the criteria as three functions to be minimized. The optimization functions $\Phi_{s}, \Phi_{f}$ and $\Phi_{a}$ corresponds to minimum clusters, minimum intra-cluster distances and minimum inter-cluster similarity respectively. The optimization functions are defined as follows:

$$
\Phi_{s}(C)=\frac{|C|}{N}
$$

where $|C|$ is the total number of clusters and $N$ is total number of documents in clustering solution $C$. The main motivation behind this objective function was to favor the clustering solutions that have small number of clusters.

$$
\Phi_{f}(C)=1-\frac{\sum_{c \in C} \frac{\left|\bigcap_{d \in c} \mathcal{F}(d)\right|}{|F|}}{|C|}
$$

where $c$ is a cluster in clustering solution $C, d$ is a document in cluster $c$, $\mathcal{F}(d)$ is the set of features in document $d$ and $|F|$ is the total number of features in clustering solution $C$. The main motivation behind this objective function was to favor the clustering solution that have compact clusters.

$$
\Phi_{a}(C)=\frac{\sum_{c, c^{\prime} \in C \wedge c \neq c^{\prime}} \delta\left(c, c^{\prime}\right)}{|C|(|C|-1)}
$$


where $c$ and $c^{\prime}$ are the clusters in the clustering solution $C$, and $\delta$ is a similarity function that computes the shared number of features between all the documents of cluster $c$ and $c^{\prime}$. The main motivation for this objective function was to favor the distinct clusters in a clustering solution.

$$
\delta\left(c, c^{\prime}\right)=\frac{\left|\bigcap_{d \in c \cup c^{\prime}} \mathcal{F}(d)\right|}{|F|}
$$

where $d$ represents a document in either cluster $c$ or $c^{\prime}$. The denominator in equation 6.3, 6.4 and 6.5 ensures that the objective function always gives values from 0 to 1 . Since we are minimizing the all three objective functions our fitness function becomes a minimization problem and can be formulated as:

$$
\mathcal{C}=\underset{C \in P}{\operatorname{argmin}}\left\{\Phi_{s}(C), \Phi_{f}(C), \Phi_{a}(C)\right\}
$$

where $C$ is a candidate clustering solution. $P$ is a set of candidate clustering solutions.

\subsubsection{Crossover}

In the crossover step, the child clustering is constructed from a pair of clustering solutions from the current population. Pairs of parents are randomly selected from the population, then a subset of clusters from each parent is randomly selected to form the child. When each cluster is selected, it is checked against the feasibility criterion to ensure that it has less than $40 \%$ overlap with any previously selected cluster. Infeasible clusters (which have more than $40 \%$ of overlap) are rejected. Clusters continue to be selected for a child until all documents are in at least one cluster of the child. If parents are exhausted before all documents are covered, the algorithm will add additional parents. 


\subsubsection{Algorithm: MMOEA}

The algorithm MMOEA developed in this research work solves the multiple objectives without combining them into a single objective function. The algorithm ranks individuals at each generation based on Pareto ranking and has the following features:

- it is based on the elitist principle.

- it implements a mechanism for explicitly preserving the diversity of solutions.

- it finds non-dominated solutions for multi-objective optimization problems.

Following are the important definitions [59]:

Definition 1. (Pareto Dominance) Let $\mathcal{Z}$ be a multi-objective optimization problem of the form: $p^{*}=\operatorname{argmin}_{p}\left\{f_{1}(p), \ldots f_{n}(p)\right\}$. Let $p^{\prime}$ and $p^{\prime \prime}$ be two candidate solutions of $\mathcal{Z} . p^{\prime}$ dominates $p^{\prime \prime}\left(p^{\prime} \prec p^{\prime \prime}\right)$ if the value of $p^{\prime}$ is lower than that of $p^{\prime \prime}$ according to at least one objective function and is less than or equal to the remaining objective functions.

Definition 2. (Pareto non-dominated set) Let $\mathcal{Z}$ be a multi-objective optimization problem of the form: $p^{*}=\operatorname{argmin}_{p}\left\{f_{1}(p), \ldots f_{n}(p)\right\}$. Let $\mathcal{X}$ be a population of individuals for $\mathcal{Z}$, i.e a set of candidate solutions of $\mathcal{Z}$. $\mathcal{X}_{\mathcal{Z}}^{*} \subseteq \mathcal{X}$ is a Pareto non-dominated solution set of $\mathcal{Z}$ w.r.t $\mathcal{X}$ if and only if $p \nprec p^{*}, \forall p \in \mathcal{X}, \forall p^{*} \in \mathcal{X}_{\mathcal{Z}}^{*}$

Definition 3. (Pareto ranking) Let $\mathcal{Z}$ be a multi-objective optimization problem of the form: $p^{*}=\operatorname{argmin}_{p}\left\{f_{1}(p), \ldots f_{n}(p)\right\}$. Let $\mathcal{X}$ be a population of individuals for $\mathcal{Z}$. The Pareto ranking function $\sigma: \mathcal{X} \rightarrow \mathbb{N}^{+}$for $\mathcal{Z}$ is defined iteratively as follows. Let $\mathcal{X}_{1}=\mathcal{X}$. For any given set of individuals $\mathcal{X}_{i}$, the Pareto rank of any $p$ belonging to the maximal Pareto non-dominated solution set $\mathcal{X}_{\mathcal{Z}, i}^{*}$ of $\mathcal{Z}$ w.r.t. $\mathcal{X}_{i}$ defined to be $i$ (i.e., $\sigma(p)=$ $i, \forall p \in \mathcal{X}_{\mathcal{Z}, i}^{*}$ ), and $\mathcal{X}_{i+1}=\mathcal{X}_{i} \backslash \mathcal{X}_{\mathcal{Z}, i}^{*}$. 
The Pareto ranking function formally described in Definition 3 provides a ranking (i.e., a score) of all solutions in a given population $\mathcal{X}$. The remaining solutions are ranked iteratively by considering the nondominated solutions which do not have a rank. This means all non-dominated solutions in $\mathcal{X} \backslash \mathcal{X}_{\mathcal{Z}, 1}^{*}$ have rank 2 and all the non-dominated solutions in $\left(\mathcal{X} \backslash \mathcal{X}_{\mathcal{Z}, 1}^{*}\right) \backslash \mathcal{X}_{\mathcal{Z}, 2}^{*}$ have rank 3 and so on. The iterative process will continue ranking until a rank (or score) is assigned to all the solutions in $\mathcal{X}$.

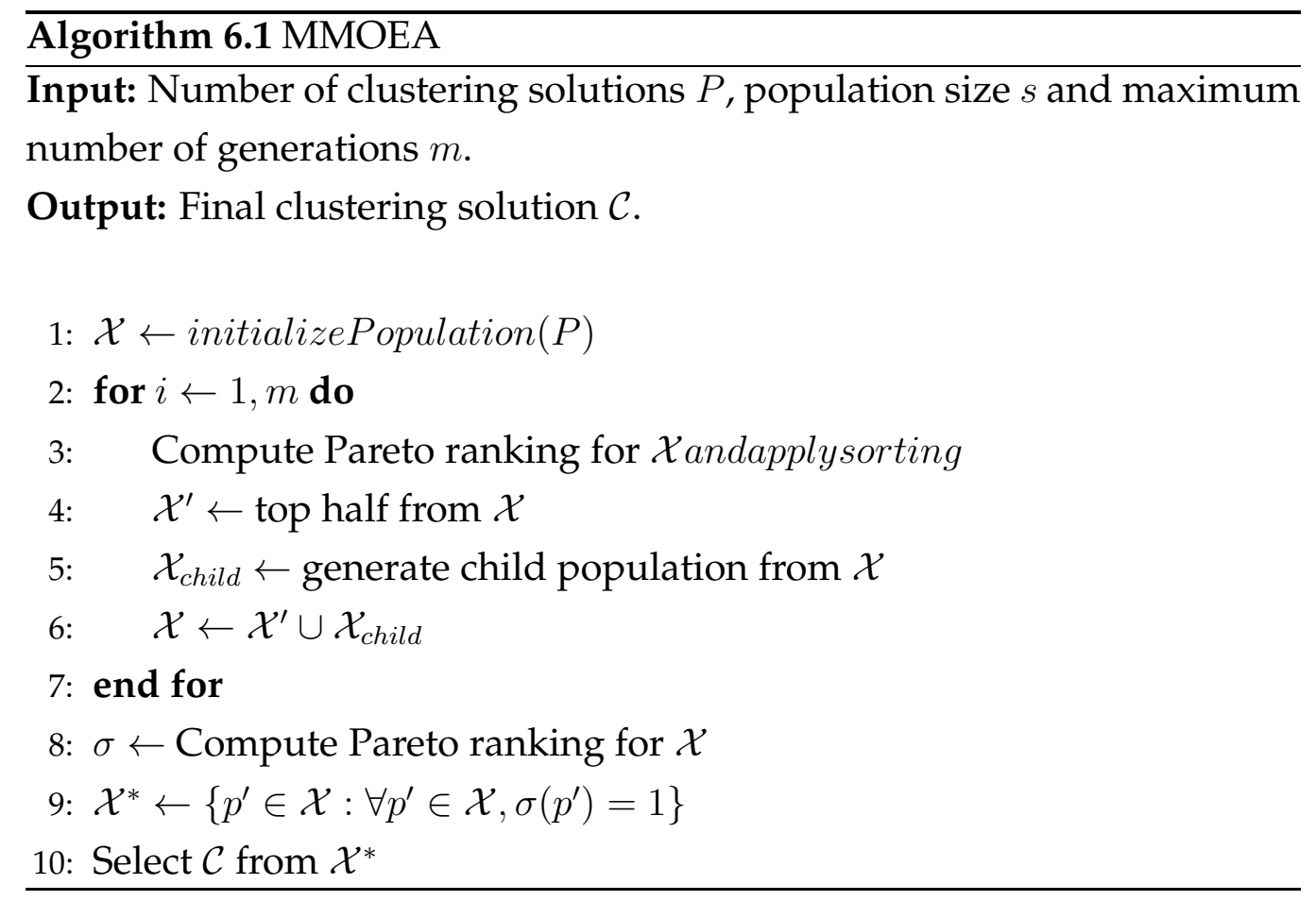

Algorithm 6.1 describes the high level operation of our algorithm MMOEA. It takes three arguments, the initial clustering solutions, the population size and the number of generations. The initial population is the set of candidate solutions $\mathcal{X}$ described in section 6.3.1 and using crossover to double the population size from 24 to 48 individuals. The loop specified on line 2 is repeated until the maximum number of iterations $m$ is reached. In each iteration the Pareto ranking function $\sigma$ is computed for current population $\mathcal{X}$ according to Definition 3 using the multi-objective function described 
in equation 6.7.

The ranking function $\sigma$ is used to sort the candidate solutions in $\mathcal{X}$ and a top half $\mathcal{X}^{\prime}$ is generated. $\mathcal{X}$ goes under a crossover to generate a new generation $\mathcal{X}_{\text {child }}$. A new population is formed by combining the set of candidate solutions of $\mathcal{X}^{\prime}$ and $\mathcal{X}_{\text {child }}$.

The set of Pareto optimal solutions $\mathcal{X}^{*}$ is computed from $\mathcal{X}$ once the loop is completed. Lastly, the final clustering solution $\mathcal{C}$ is randomly selected from rank 1 Pareto front. Please note that there are better ways to select the final clustering solutions like using crowding distance, however, our main focus was in improving the clustering process instead of improving the genetic search.

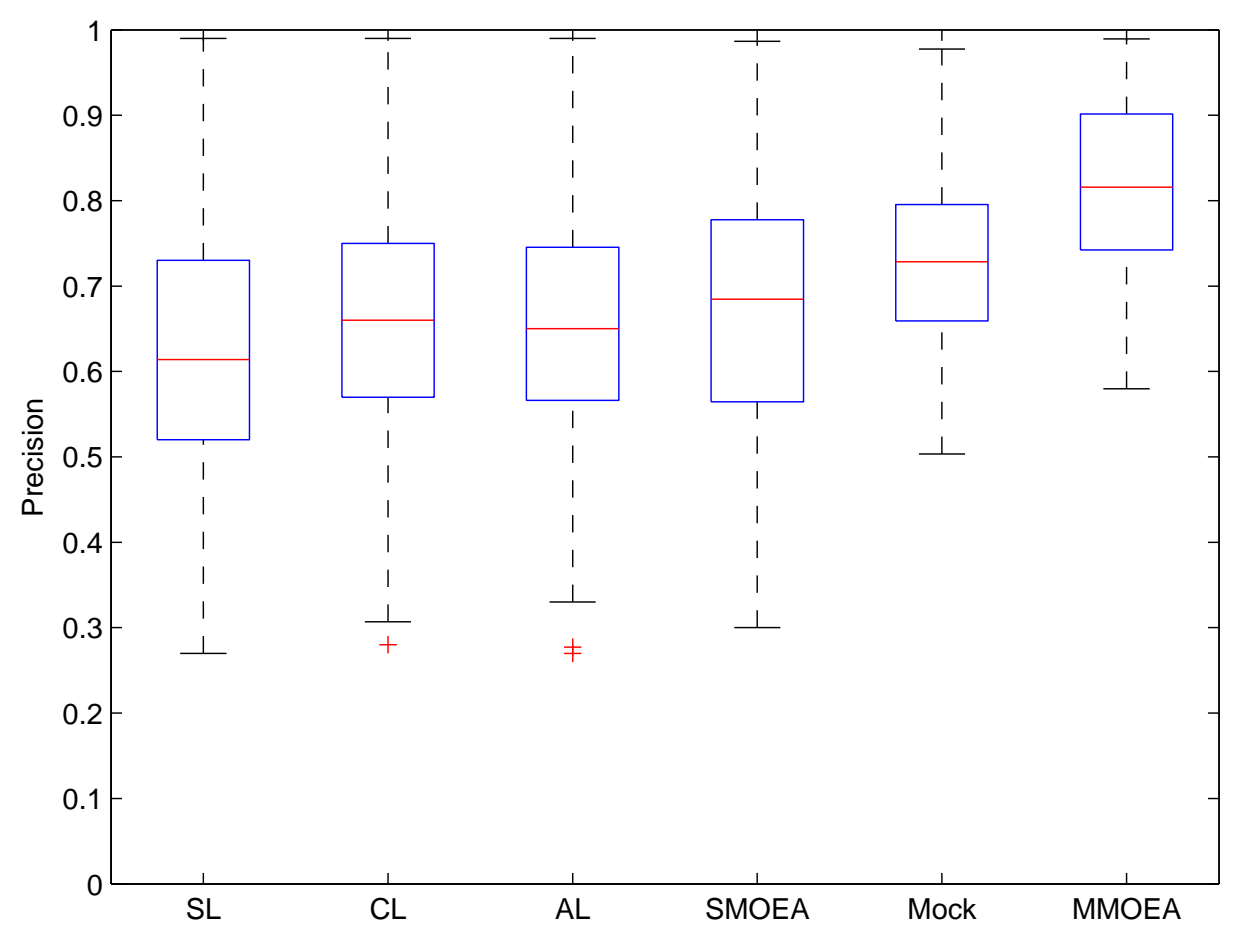

Figure 6.4: Precision of the final solution on all datasets 


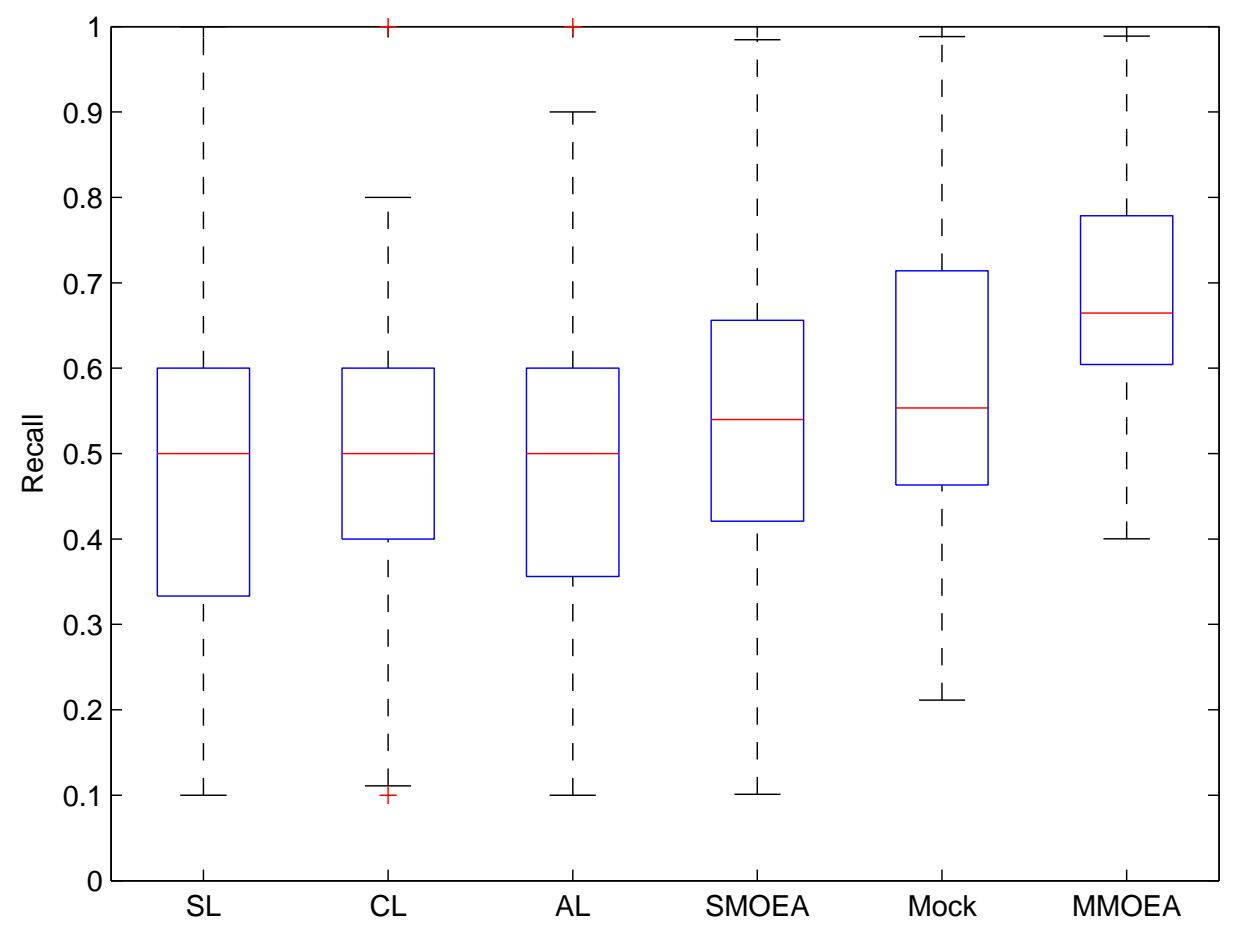

Figure 6.5: Recall of the final solution on all datasets

\subsection{Evaluation Experiments}

Our multi-view ensemble method was compared with five existing clustering methods by evaluating the quality of the clusters against the gold standard provided by the datasets. We used F-measure scores [30] and Rand Index (RI) values [70] for evaluation ${ }^{4}$.

The datasets used for evaluation are $\mathrm{AMBIENT}^{5}, \mathrm{MORESQUE}^{6}$ and ODP-2397, which has different levels of difficulty in finding the clusters. The details of the datasets and evaluation measures are provided in chapter 3

\footnotetext{
${ }^{4}$ Note that the evaluation measures are necessarily different from the objective functions, because the gold standard cannot be made available to the clustering system

${ }^{5}$ downloaded from http://credo.fub.it/ambient/

${ }^{6}$ downloaded from http://lcl.uniroma1.it/moresque/

${ }^{7}$ downloaded from http://credo.fub.it/odp239/
} 


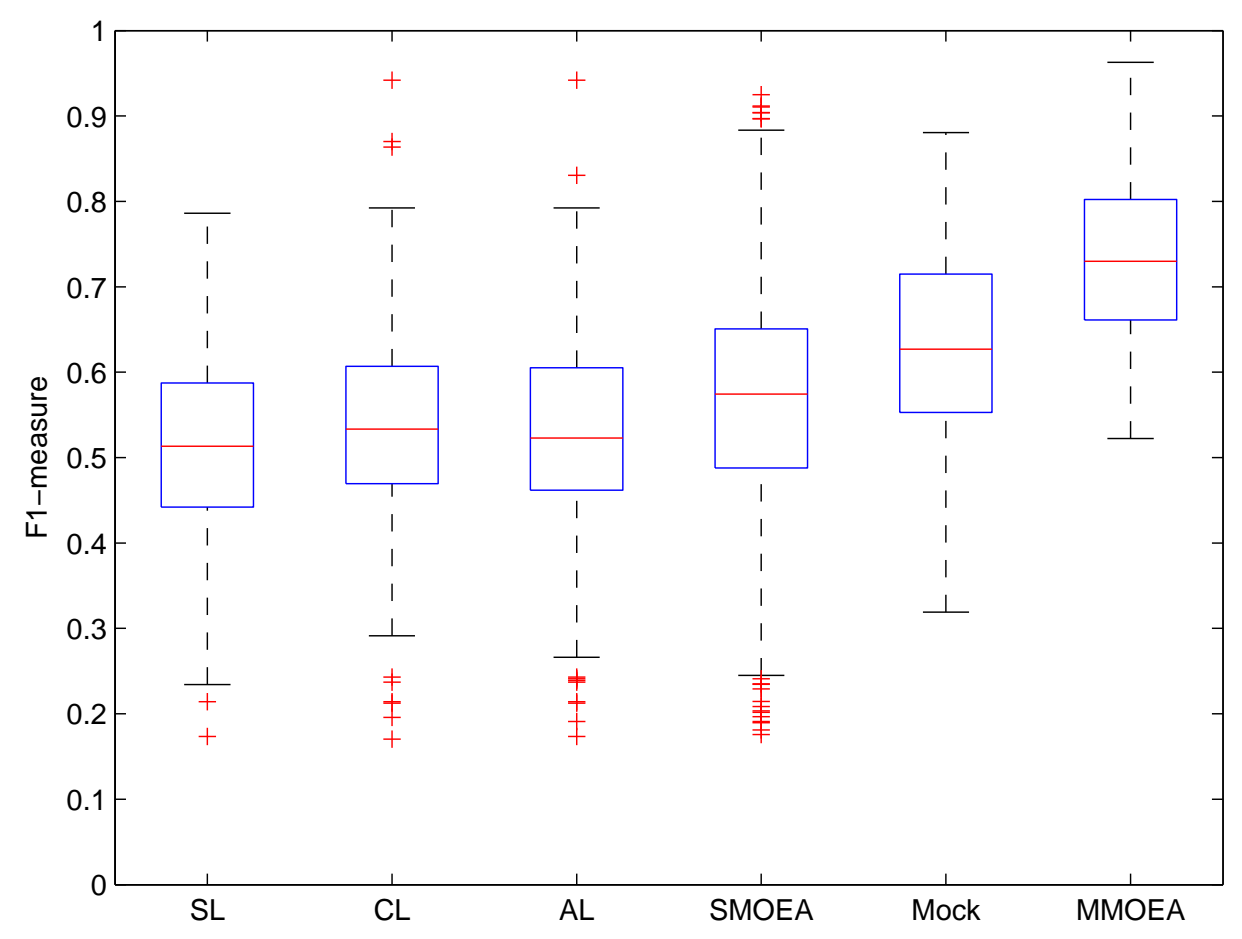

Figure 6.6: F1-measure on all datasets

Our method MMOEA was compared with five methods: Single Link (SL), Complete Link (CL), Average Link (AL), Single-view MOEA (SMOEA) and Mock. In our method MMOEA, the total population size was 24 and maximum number of generations was 1000 .

We implemented SL, CL and AL as three single objective clustering ensemble methods based on link-based pairwise similarity matrix known as Approximate SimRank-based similarity matrix (ASRS) [72] which is a modified version of SimRank-based similarity matrix (SRS) [71]. For the step 1 of SL, CL and AL, we generated candidate clustering by applying 10 different initializations with fixed number of clusters of k-means algorithm on one feature matrix (term view with tfidf scheme only). For Step 2 ASRS matrix was generated from the results of k-means algorithm, and then in order to generate a final clustering solutions then we applied single-link hierarchical clustering for SL; complete-link hierarchical clus- 


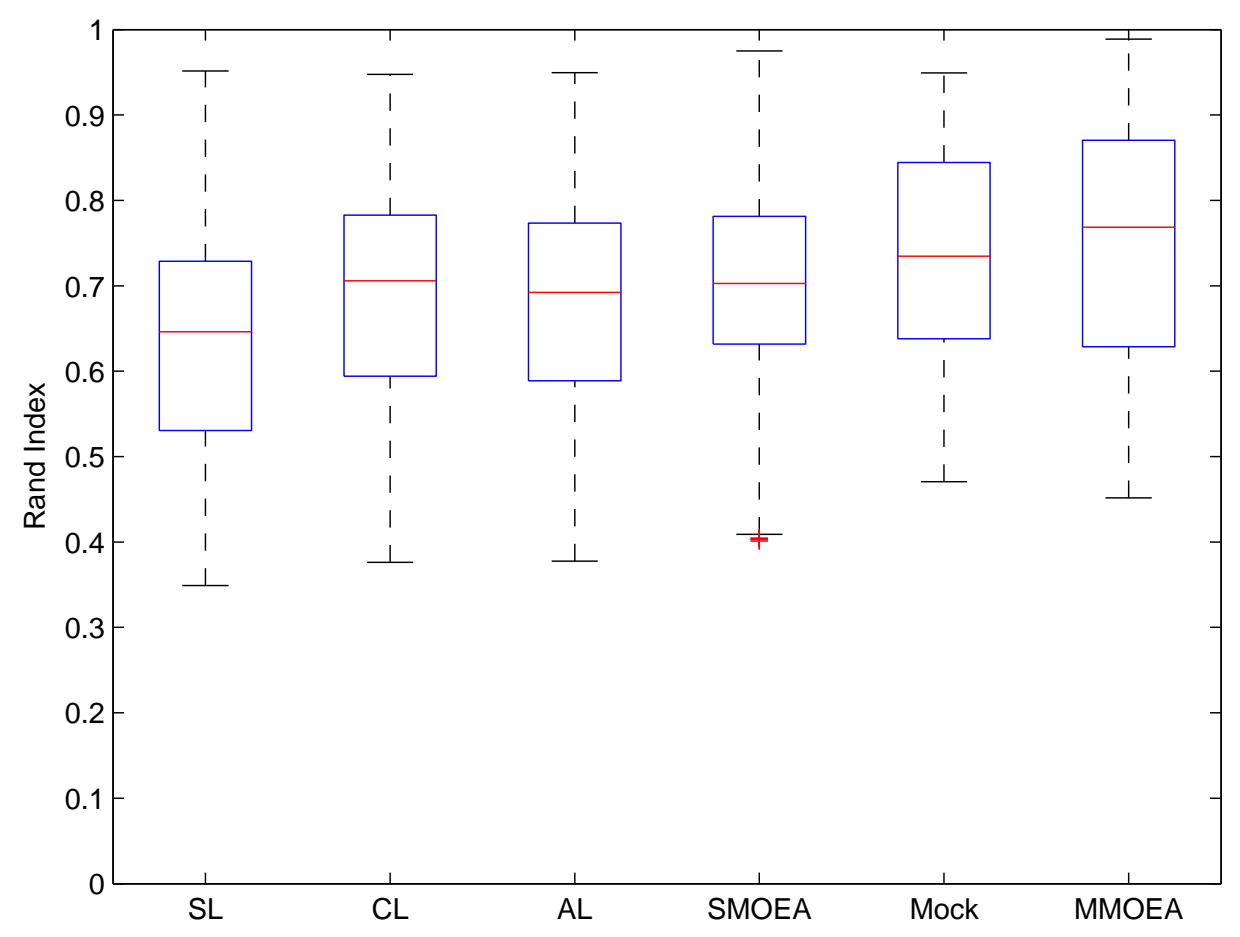

Figure 6.7: Rand Index on all datasets

tering for CL; and average-link hierarchical clustering for AL on ASRS matrix.

SMOEA is a single view version of our algorithm MMOEA and uses only the term view (tfidf view) with the same GA parameters as MMOEA. Mock is the state-of-the-art multi-objective evolutionary algorithm ${ }^{8}$ [62] and was used with standard parameters (code was provided by the author).

\subsection{Results and Discussion}

Figures 6.4, 6.5, 6.6 and 6.7 display boxplots of precision, recall, F1-measure and RI values computed for all the queries (397 queries in total where each

${ }^{8}$ http:/ / personalpages.manchester.ac.uk/mbs/julia.handl/mock.html 
query corresponds to 100 documents that are required to be clustered) in all three datasets using Single Link Agglomerate Hierarchical Clustering Ensemble (SL), Complete Link Agglomerate Hierarchical Clustering Ensemble (CL), Average Link Agglomerate Hierarchical Clustering Ensemble (AL), Mock and our methods (SMOEA and MMOEA). The y-axis represents the score from 0 to 1 and $x$-axis represents the clustering methods. The mean value is marked by a line in the middle of the box and the box represents the quartiles ( $25 \%$ to $75 \%$ ) of the values. The lower and upper bound of the dashed line on the box represents the deviation (5\% to 95\%) of the values and the rest of the values are marked as plus signs (generally considered as outliers).

Figures 6.4, 6.5, 6.6 and 6.7 show that the mean values of MMOEA in terms of Precision, Recall, F1-measure and RI are significantly higher. SMOEA had better mean values than SL, CL and AL but worse than Mock and MMOEA in all experiments.

The spread of Precision, Recall and F1-measure show that MMOEA is much better, however in terms of RI, MMOEA is comparatively better than Mock and much better than other methods. SMOEA produced better results according to F1 and RI measure than SL, CL and AL, however its results were not better than Mock, and MMOEA. The experiments on individual datasets (AMBIENT, MORESQUE and ODP239) also showed similar results.

\subsubsection{Statistical Analysis}

Statistical analysis was performed to draw a precise conclusion from the results of F1-measure and RI. Table 6.1 shows a ranking of clustering methods computed on F1 and RI values on all datasets based on Friedman's method [49]. It is evident that MMOEA is at the top with the ranking of 2.1511 and 2.1738 in terms of F1-ranking and RI-ranking respectively. Mock is at second place with ranking of 2.4169 and 2.7960. SMOEA se- 


\begin{tabular}{|l|c|c|}
\hline Method & F1-Ranking & RI-Ranking \\
\hline MMOEA & 2.1511 & 2.1738 \\
\hline Mock & 2.4169 & 2.7960 \\
\hline SMOEA & 2.8249 & 3.1008 \\
\hline Complete Link & 3.3249 & 3.1998 \\
\hline Average Link & 3.4005 & 3.2821 \\
\hline Single Link & 4.5466 & 3.6474 \\
\hline
\end{tabular}

Table 6.1: Average ranking of clustering methods on F1-measure and RI values

cured third place with ranking of 2.8249 and 3.1008 in terms of F1-ranking and RI-ranking respectively. Friedman's measure, $\chi_{F}^{2}$, for 4 degrees of freedom is 648.03 for F1 values and 198.1019 for RI values. These values signify that F1 and RI values are not random (observed by considering the critical values) and these results are statistically significant.

Since our interest was to compare MMOEA with other methods, we took F1 values and RI values of MMOEA as control group separately and performed the Bonferroni-Dunn test for $\alpha=0.05$. The $p$-values were significantly lower than 0.0001 which indicated that MMOEA is significantly better than others.

It is interesting to know how using multiple views in clustering methods can affect the clustering results. We did more experiments by using the multiple views in single objective clustering ensembles (SL, CL and $\mathrm{AL}$ ) and observed that results were almost as good as the original version of SL, CL and AL but worse compared to Mock, SMOEA and MMOEA. Using multiple views will result in diverse clusters in different candidate clustering solutions. However directly applying standard algorithms is not enough to achieve better performance. Generating diverse clusters and choosing the high quality clusters from candidate clustering solutions, both in conjunction results in a better clustering approach. 


\subsection{Summary}

This chapter has presented a multi-objective approach for clustering ensembles (MMOEA) that uses multiple views to generate a set of candidate solutions and selects high-quality overlapping clusters from the candidate solutions to form a superior clustering solution. This chapter has three contributions to improve clustering results. The first contribution is to use multiple views to generate an initial set of candidate clustering solutions. This results in diverse candidate clustering solutions having mixtures of high quality and low quality clusters. The second contribution is to make a new final clustering solution by combining the individual clusters from different candidate clustering solutions. The third contribution is to use the multi-objective ranking system from NSGA-II to guide the optimization because our objective criteria are in conflict with each other.

The experiments have shown that MMOEA outperformed other methods in terms of F1 and RI index. SMOEA, the single view version of MMOEA, was better than the single objective methods but as it did not have a diverse set of clusters in step 1 of the clustering algorithm, it was not able to produce a better result than Mock or MMOEA. Therefore we conclude that having a diverse set of clusters from multiple meaningful views plays an important role in our approach.

MMOEA is limited to domains that can have overlapping clusters and whose data can be represented by multiple views. Since documents can naturally be categorized under different topics and can be represented from different views, this work can be applied to various collections of documents (corpus). The only modification required is in the method which generates the matrix. For example, in the case of the Reuters data set in which there are no queries, we would just disable the user query sense and use only two views (topics and terms).

In next chapter we will present another multi-objective approach (MOMVEC) which is an improved version of this approach that has better crossover, 
mutation operators and objective functions. 


\section{Chapter 7}

\section{Improving Multi Objective Multi-View Ensemble Clustering}

\subsection{Introduction}

Recently, evolutionary approaches have become popular for multi-objective clustering ensembles because of their ability to provide good results [62], but evolutionary algorithms depend heavily upon the choice of evolutionary operators (such as selection, crossover, mutation etc.) and the fitness evaluation criteria which are both domain specific. In previous chapter we discussed a Multi-view Multi-Objective Evolutionary Algorithm (MMOEA) [132] which uses multiple views to generate an initial set of candidate clustering solutions and then applies an evolutionary approach (NSGA-II) using a simple crossover method and three objective functions to select better quality clusters. MMOEA has several limitations. The simple crossover method might not result in diverse clustering solutions and hence needs to be replaced by better crossover functions. MMOEA has no mutation step, but mutation and tuning steps could help in exploring interesting search space and give faster convergence.

Köppen et.al, argued that NSGA-II is not suitable for solving many (more than two) objective optimization problems [80]. Therefore the use 
of three objective functions is questionable and it would be better to either use other multi-objective algorithms or reduce the number of objectives.

The main motivation for this chapter was to address the limitation in MMOEA and provide a comparison of SPEA-II and NSGA-II multi objective optimization approaches with respect to clustering problem.

This chapter introduces two new approaches of clustering ensembles: Multi-objective Document clustering (MDC) based on SPEA-II and MultiObjective Multi-View Ensemble Clustering (MOMVEC) based on NSGAII. MOMVEC is a variation of MDC and the main difference between the two methods is the evolutionary approach. The following are the key innovations for these two methods.

1. Developing more sophisticated crossover methods for generating new clusters for candidate clustering solutions.

2. Developing mutation methods for splitting and merging clusters.

3. Developing a two objective fitness function for multi-objective clustering ensembles.

Section 7.2- 7.4 presents MDC and MOMVEC methods; section 7.6 describes the experimental setup and presents the results for MDC; section 7.7 describes the experimental setup for MOMVEC; section 7.7.1 provides the experiment results of MOMVEC with discussions and lastly section 7.9 provides a summary of the chapter.

\subsection{Common Elements of MDC and MOMVEC}

This section describes the common steps of MDC and MOMVEC. Just like MMOEA, both methods exploit multiple views of documents to generate various candidate clustering solutions in step 1 of the clustering process 
and then form a final clustering solution by combining a set of high quality clusters from different candidate clustering solutions using an evolutionary process. The details of MMOEA are given in previous chapter.

MDC and MOMVEC use two criteria based on intra-cluster and intercluster distances to evaluate the fitness of individuals and perform Selection, Crossover, Mutation and Tuning steps on the population to generate a new set of candidates. The difference between the two algorithms is the method for finding the Pareto fronts and the evolutionary approach for selecting final clusters. The initialization and genetic representation for MDC and MOMVEC are described in chapter 6 . The rest of this section describes the common components of the two methods.

\subsubsection{Fitness Evaluation}

The fitness evaluation of candidate clustering solutions is based on the following two objective criteria.

1. Intra-cluster distance: Minimize the distances between the objects within each cluster of a clustering solution.

2. Inter-cluster similarity: Minimize the similarity between each pair of clusters in a clustering solution.

The first objective is strongly biased towards small clusters (e.g., singletons will have minimum intra-cluster distance) whereas the second objective is less biased towards small clusters. We added a weighting parameter $\left(\frac{1}{\sqrt{|c|}}\right)$ based on cluster size $(|c|)$ in the first objective function to penalize the small clusters and favor a clustering solution with big clusters. We used $\sqrt{|c|}$ instead of $|c|$ to reduce the impact of cluster size.

Now the two objectives trade off against each other. The first objective gives preference to clustering solutions that have big clusters of closely related objects and the second objective gives preference to small clusters that are different from each other. 
The first objective function is defined as follows:

$$
\Phi_{a}(C)=\frac{1}{|C|} \sum_{c \in C} \frac{1}{\sqrt{|c|}}\left(\frac{1}{|c|(|c|-1)} \sum_{d, d^{\prime} \in c} \delta\left(d, d^{\prime}\right)\right)
$$

where $c$ is a cluster in clustering solution $C, d$ and $d^{\prime}$ are documents in cluster $c$, and $\delta$ is a distance function which computes the distance between two documents. $\Phi_{a}(C)$ is the average intra-cluster distance of all clusters weighted by inverse square root of the cluster size $\frac{1}{\sqrt{|c|}}$. The total number of clusters in clustering solution $|C|$ was used as a denominator to ensure that the value of $\Phi_{a}(C)$ ranges from $0-1$.

We used cosine similarity for computing the distances between the documents where $\delta\left(d, d^{\prime}\right)=1-\gamma\left(d, d^{\prime}\right)$, where $\gamma\left(d, d^{\prime}\right)$ represents the cosine similarity between $d$ and $d^{\prime}$.

The second objective function computes the average similarity of clusters in a clustering solution where the similarity between two clusters is the average similarity of the pairs of documents in the two clusters. The second objective function is defined as follows:

$$
\Phi_{e}(C)=\frac{1}{|C|(|C|-1)} \sum_{c, c^{\prime} \in C \wedge c \neq c^{\prime}}\left(\frac{1}{|c|\left|c^{\prime}\right|} \sum_{d \in c, d^{\prime} \in c^{\prime}} \gamma\left(d, d^{\prime}\right)\right)
$$

where $c$ is a cluster in clustering solution $C, d$ and $d^{\prime}$ are the documents in cluster $c$, and $\gamma$ is a similarity function which computes the similarity between two documents. $\Phi_{e}(C)$ is the average similarity over all pairs of clusters and ranges from $0-1$.

Please note that the above mentioned objective criteria are not suitable for a clustering solution which only has one cluster. Our algorithms do not produce clustering solutions with only one cluster.

Both objective functions are coded in such a way that their values are required to be minimized. Therefore our algorithm must solves the multiobjective optimization problem which attempts to find a solution that minimizes both $\Phi_{a}(C)$ and $\Phi_{e}(C)$ objective functions. 
The evolutionary approach not only requires a good fitness evaluation criteria but also needs mechanisms to generate new and diverse candidate clustering solutions in order to avoid local optima.

\subsubsection{Selection}

The selection step selects a number of candidate solutions from the previous iteration to generate a set of new candidate clustering solutions. We used selection method based on SPEA-II for MDC [155] and selection method based on NSGA-II for MOMVEC [36].

\subsubsection{Crossover}

In evolutionary approaches, crossover is a process for generating a new individual from two previous individuals. MDC and MOMVEC used two crossover methods: a row-wise method and a column-wise crossover method (rows represent clusters and columns represent documents).

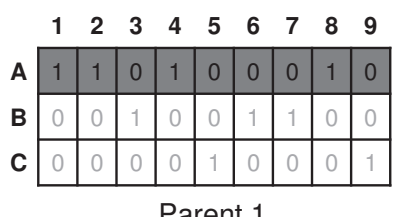

Parent 1

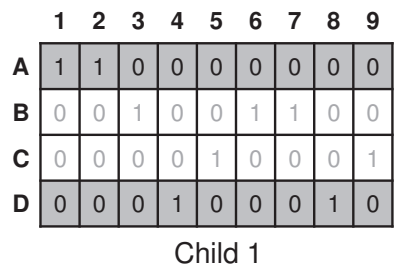

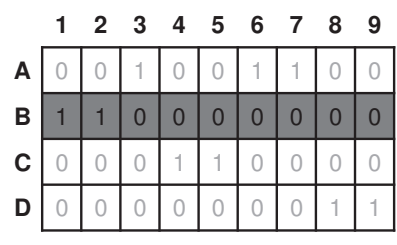

Parent 2

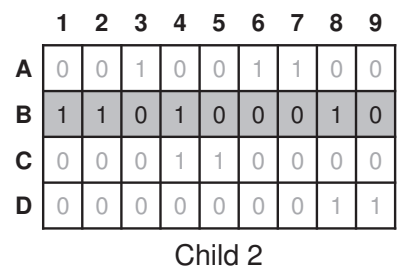

Figure 7.1: Example of row-wise crossover method.

The row-wise method swaps a randomly chosen row from one parent with a randomly chosen row from the other parent, creating two new children. If either child has any columns that are all zeros (i.e. unassigned 
documents), it adds an extra row to that child containing these documents. This crossover method may result in overlapping clusters but the coverage will always be $100 \%$.

Figure 7.1 shows an example of the row-wise crossover method in which the rows with dark gray background are swapped between Parent 1 and Parent 2 and as a result Child 1 and Child 2 are created. The extra row highlighted in light gray in Child 1 contains the unassigned documents after the swapping.
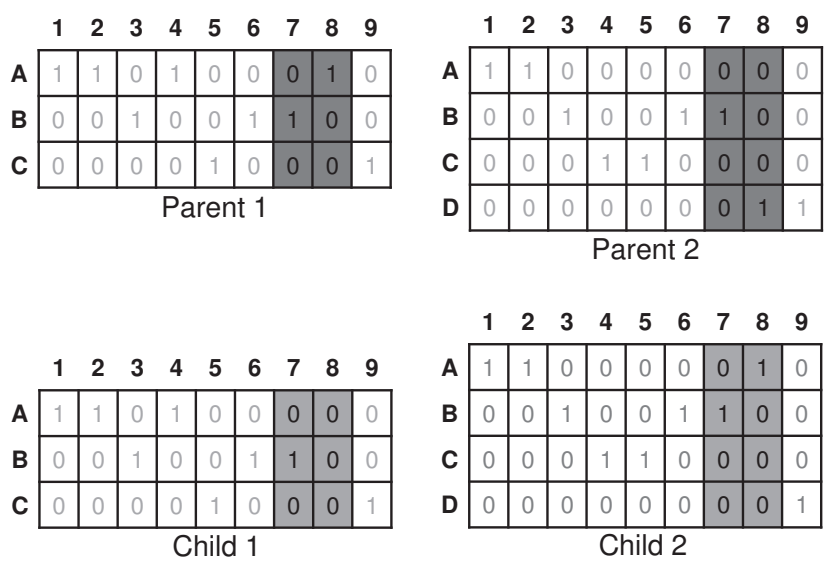

Figure 7.2: Example of column-wise crossover method.

The column-wise method swaps (from 2 to 9) randomly chosen columns of one parent with the corresponding columns of the other parent. If the parents have different number of clusters, then excess rows are given zero values for chosen columns. An additional step in the column-wise crossover is to remove empty clusters. This type of crossover does not affect the overlapping of documents but may result in less than $100 \%$ coverage.

Figure 7.2 shows an example of the column-wise crossover method in which the columns with dark gray background are swapped between Parent 1 and Parent 2 and as a result Child 1 and Child 2 are created. Column 8 in Child 1 contains a zero value for all rows indicating that the document was not assigned to any cluster. 

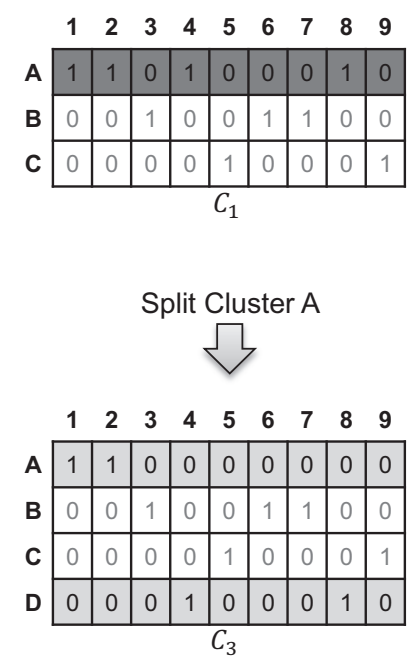

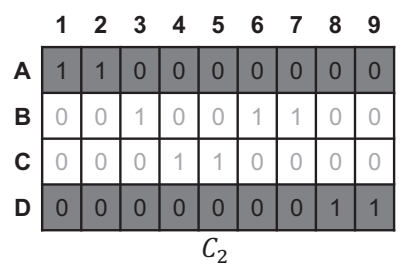

Merge Cluster $\mathrm{A}$ and $\mathrm{D}$
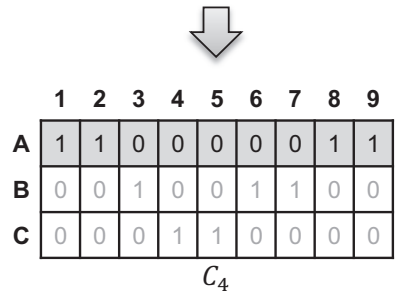

Figure 7.3: Example of split and merge mutation method.

\subsubsection{Mutation}

MDC and MOMVEC use two different types of mutation methods. Splitmutation splits big clusters into two clusters and merge-mutation merges two small clusters into one cluster. We used a random approach for splitmutation which forms two clusters randomly from one cluster. The mergemutation is based on inter-cluster distances and merges the two small clusters in a candidate clustering solution that have the minimum inter cluster distance.

Figure 7.3 shows two examples of mutation. On the left, split-mutation is applied to $C_{1}$ to generate $C_{3}$. Cluster (row) $\mathrm{A}$ is randomly selected and split into two clusters A and D. On the right, merge-mutation is applied to $C_{2}$. Clusters $\mathrm{A}$ and $\mathrm{D}$ in $C_{2}$ are merged into cluster $\mathrm{A}$ in $C_{4}$.

\subsubsection{Tuning}

The newly generated candidate clustering solutions are mostly based on a random approach and may not represent sensible clusterings. This would cause the evolutionary approach to converge slowly. Therefore we need 
a local search mechanism to find local optima more quickly. This tuning is based on k-means and includes the following two steps for all newly generated candidate clustering solutions.

1. Calculating cluster centroids.

2. Relocating each document to the cluster with the nearest centroid.

These two steps are repeated until there is no change to centroids and no more relocation of documents.

\subsection{Multi-objective Document Clustering (MDC)}

The algorithm that MDC uses for finding Pareto fronts and selecting candidate clustering solutions is based on SPEA-II.

Algorithm 7.1 shows the process of our new method MDC. The algorithm is a modified version of SPEA-II and starts with the initial population, which is generated by exploiting the multiple views of the documents. It computes the objective functions $\Phi_{a}(C)$ and $\Phi_{e}(C)$ for each individual in a population. Then, using the SPEA-II method, we identify the non-dominated solutions and finally perform our selection, crossover, mutation and tuning steps (as described earlier) on the current population to generate a new population. The truncation operator, mentioned in the algorithm, removes $50 \%$ of the worst individuals in a population by considering their fitness value. The stopping criterion for the algorithm is the total number of generations.

\subsection{Multi-objective Multi-View Ensemble Clus- tering (MOMVEC)}

MOMVEC, on the other hand, is based on NSGA-II. Figure 7.4 presents an overview of MOMVEC. The first step of the MOMVEC method is to 


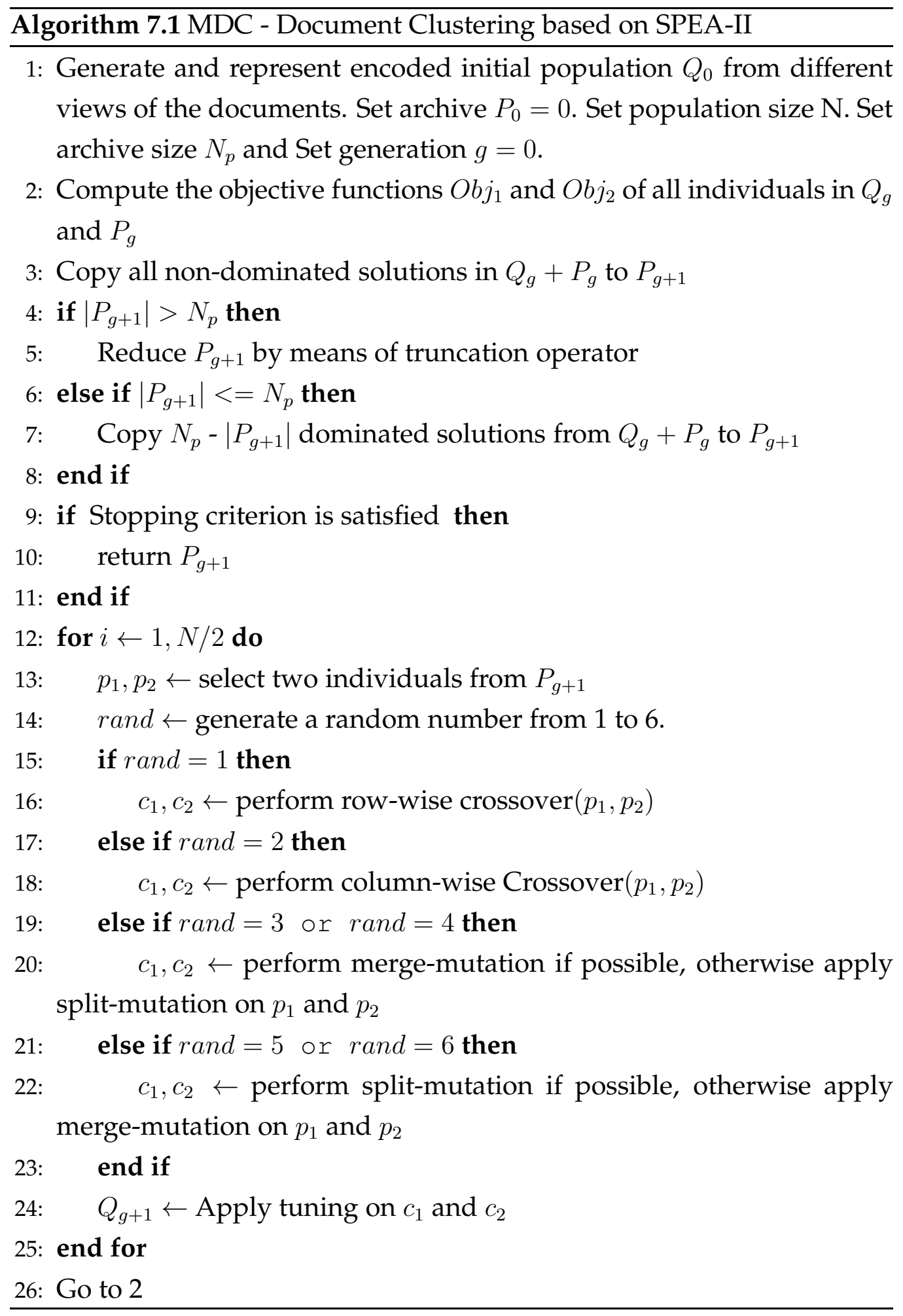


identify multiple views of the dataset. Then these views are used to generate multiple feature matrices to which clustering algorithms are applied to generate multiple candidate clustering solutions. These initial candidate solutions are then encoded in the genetic representation and form the initial population, and their fitness is evaluated on the basis of two criteria.

The evolutionary process is then applied. The current population consists of $Q$ (the set of new individuals just created) and $P$ (the elite individuals obtained from the previous iteration).

After fitness evaluation on $Q$, we use NSGA-II to compute Pareto fronts (F1, F2 etc.) and rank all the candidate clustering solutions (i.e. $P$ and $Q$ ). If the stopping criterion is not met then we retain the top half of the ranked candidate solutions of $\mathrm{P}$ and $\mathrm{Q}$, and then perform Selection, Crossover, $\mathrm{Mu}$ tation and Tuning steps to generate a set of new candidate clustering solutions $Q$.

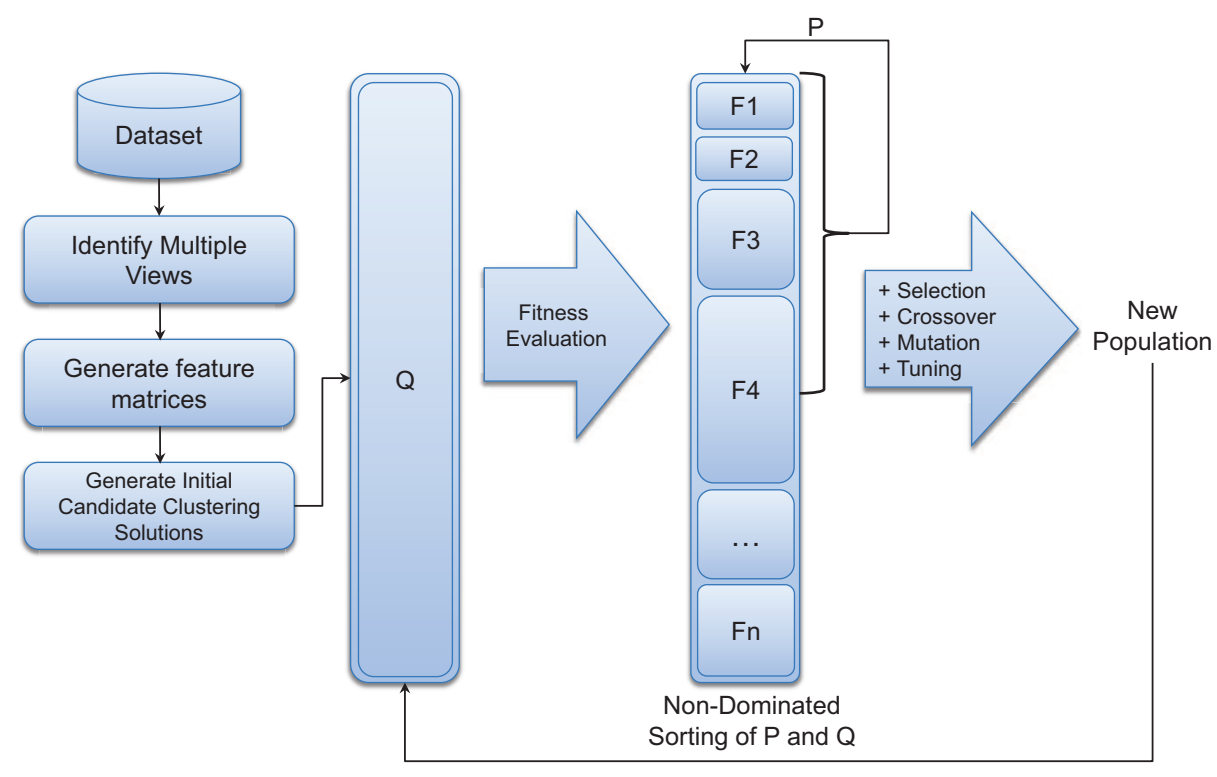

Figure 7.4: Overview of MOMVEC clustering method

The algorithm MOMVEC, uses a multiple objectives approach for selecting the final clustering solution. Similar to NSGA-II, the algorithm 


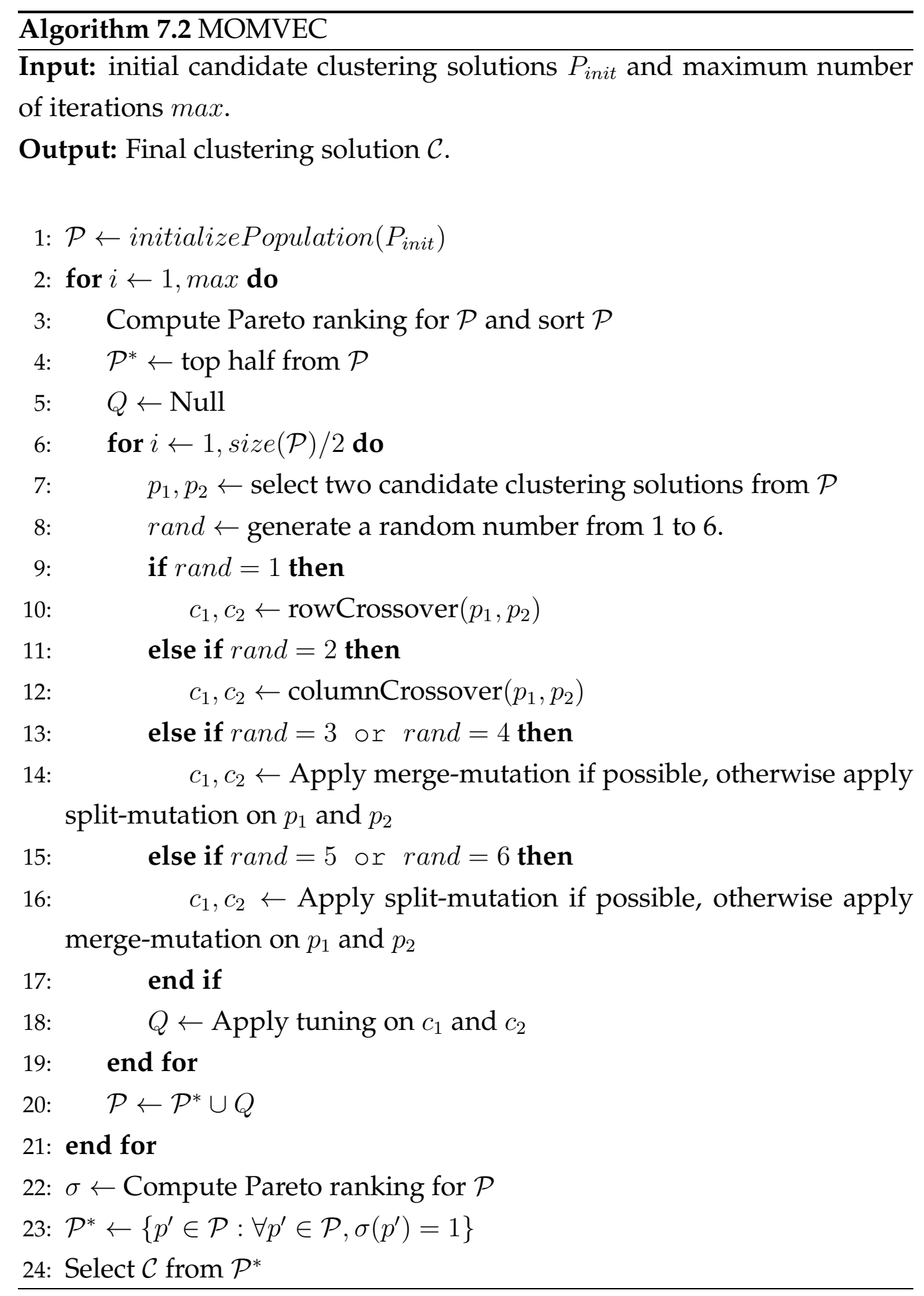


ranks individuals iteratively based on Pareto ranking and crowding distance.

Algorithm 7.2 outlines the operation of our algorithm MOMVEC. It takes two arguments as inputs, the initial candidate clustering solutions and the maximum number of iterations. The initial candidate clustering solutions are the set of candidate solutions $\mathcal{P}$ generated from multiple views (as described in section 6.3.1). The Pareto ranking function $\sigma$ is calculated for the current set of candidate clustering solutions $\mathcal{P}$ according to Definition 3 provided in chapter 6 using the objective functions $\Phi_{a}(C)$ and $\Phi_{e}(C)$.

The ranking function $\sigma$ sorts the candidate clustering solutions $\mathcal{P}$. The loop on line 6 generates a new set of candidate clustering solutions by applying different methods on $\mathcal{P}$. The row-wise and column-wise crossover methods are chosen with a probability of $\frac{1}{6}$ whereas the merge-mutation and split-mutation methods are chosen with a probability of $\frac{1}{3}$. The new candidate clustering solutions go under the tuning step and are then merged with previous top rank (non-dominated) set of candidate clustering solutions $\mathcal{P}^{*}$.

Once the main loop is completed, the set of Pareto optimal solutions $\mathcal{P}^{*}$ is computed from $\mathcal{P}$. The final clustering solution $\mathcal{C}$ is finally selected based on the longest crowding distance from the rank 1 Pareto front as described by [36].

The random method for selecting a final clustering solution might not result in selecting the best solution from the Pareto optimal front and the same is true for using crowding distance. However, using the crowding distance increases the chances of choosing a better solution.

Please note that there are better ways to select the final clustering solutions (e.g.[110]), however, our main focus was on clustering algorithms instead of improving the genetic search. Further discussions about crowding distance can be found at [46]. 


\subsection{Experimental Setup}

\subsubsection{Datasets}

We created eight datasets (D1-D8) from WebKB2 and WebKB4 datasets which have two predefined views of webpages ${ }^{1}$. Also, we used Citeseer 2 and Cora $^{3}$ dataset which also have two predefined views. Apart from two view datasets we used a three view dataset which is the combination of AMBIENT ${ }^{4}$, MORESQUE ${ }^{5}$ and ODP-2396 datasets. The three views of combined dataset were generated from topics, terms and ambiguous queries of the document using wikiminer toolkit [96].

\subsubsection{Evaluation Measure}

The clustering performance was evaluated using Clustering Accuracy (CA) [105], F1-measure (F1) [131, 30] and Rand Index (RI) [70]. F1-measure is defined as the weighted harmonic mean of Precision and Recall. Precision measures the accuracy of a system by considering the majority topics of the clusters and Recall measures the coverage of different topics in a clustering solution [30].

The details of the datasets and evaluation measures were provided in chapter 3.

\subsection{Experimental Setup for MDC}

The algorithm was run multiple times and the average of all the runs is reported in this research work. The parameters for the algorithms include

\footnotetext{
${ }^{1}$ Both of the datasets can be downloaded from http : / / www . cs . cmu . edu / webkb/

${ }^{2}$ can be downloaded from http: / / www. cs. umd.edu/ sen/lbc-proj/data/citeseer.tgz

${ }^{3}$ can be downloaded from http: //www. cs. umd. edu/ sen/lbc-proj/data/cora.tgz

${ }^{4}$ can be downloaded from http: / / credo. fub.it/ambient/

${ }^{5}$ can be downloaded from http: / / lcl . uniromal.it/moresque/

${ }^{6}$ can be downloaded from http: //credo. fub. it/odp239/
} 
the following: maximum number of generations was set to 1000, crossover probability was set to $\frac{1}{6}$, mutation probability was set to $\frac{1}{3}$, population size was set to 20. These parameters were fixed for all runs of the algorithm. The number of clusters were randomly chosen at the initial stage ranging from 2-10 for k-mean clustering and fixed number of clusters (predefined number of clusters from gold standard) for other clustering methods.

MDC was compared with a simple ensemble clustering method (using an average link hierarchical method as consensus function) and two multiobjective clustering approaches based on evolutionary approaches (Mock and MMOEA). The results were compared using three different evaluation metrics on different datasets. These evaluation metrics are widely used to measure the quality of clustering by comparing the clustering solutions produced by clustering methods with given gold standard clustering solution provided by the datasets ${ }^{7}$.

Table 7.1: Clustering Accuracy computed on 10 two view datasets.

\begin{tabular}{lccccccccccc}
\hline \multirow{2}{*}{ Dataset } & \multicolumn{2}{l}{ Avg-Ensemble } & \multicolumn{2}{c}{ MOCK } & \multicolumn{3}{c}{ MMOEA } & \multicolumn{3}{c}{ MDC } \\
\cline { 2 - 11 } & \multicolumn{2}{c}{$\mathrm{v} 1$} & $\mathrm{v} 1+\mathrm{v} 2$ & $\mathrm{v} 1$ & $\mathrm{v} 1+\mathrm{v} 2$ & $\mathrm{v} 1$ & $\mathrm{v1+v2}$ & 2view & $\mathrm{v} 1$ & $\mathrm{v1+v2}$ & 2view \\
\hline D1 & 83.95 & 83.95 & 84.34 & 84.43 & $\mathbf{9 1 . 1 2}$ & $\mathbf{9 1 . 1 2}$ & 91.12 & $\mathbf{9 1 . 1 2}$ & $\mathbf{9 1 . 1 2}$ & $\mathbf{9 2 . 5 1}$ \\
D2 & 86.22 & 87.94 & 87.01 & 87.39 & $\mathbf{9 0 . 1 2}$ & 90.21 & 90.42 & $\mathbf{9 0 . 1 2}$ & $\mathbf{9 0 . 2 1}$ & $\mathbf{9 1 . 4 5}$ \\
D3 & 71.09 & 71.09 & 80.12 & 81.23 & 84.91 & 86.43 & 86.72 & $\mathbf{8 7 . 0 5}$ & $\mathbf{8 8 . 1 2}$ & $\mathbf{9 2 . 1 3}$ \\
D4 & 73.83 & 74.16 & 74.98 & 75.33 & 85.54 & 86.96 & 88.54 & $\mathbf{8 8 . 1 7}$ & $\mathbf{9 0 . 1 5}$ & $\mathbf{9 2 . 8 8}$ \\
D5 & 56.41 & 57.69 & 58.01 & 59.98 & 68.12 & 68.82 & 70.12 & $\mathbf{7 0 . 1 2}$ & $\mathbf{7 1 . 1 3}$ & $\mathbf{7 5 . 2 2}$ \\
D6 & 72.36 & 60.17 & 72.36 & 60.17 & 73.01 & 74.87 & 75.45 & $\mathbf{7 4 . 1 1}$ & $\mathbf{7 6 . 8 5}$ & $\mathbf{7 8 . 1 3}$ \\
D7 & 69.13 & 69.65 & 69.82 & 70.23 & 72.17 & 72.51 & 74.17 & $\mathbf{7 2 . 3 1}$ & $\mathbf{7 2 . 5 4}$ & $\mathbf{7 7 . 9 3}$ \\
D8 & 78.11 & 78.49 & $\mathbf{7 9 . 9 1}$ & 80.45 & $\mathbf{7 9 . 9 1}$ & 80.98 & 79.91 & $\mathbf{7 9 . 9 1}$ & $\mathbf{8 1 . 4 4}$ & $\mathbf{8 3 . 9 1}$ \\
Citeseer & 43.87 & 44.32 & 45.98 & 45.98 & 50.01 & 51.45 & 54.34 & $\mathbf{5 1 . 1 2}$ & $\mathbf{5 3 . 3 2}$ & $\mathbf{6 0 . 1 2}$ \\
Cora & 44.72 & 46.35 & 46.51 & 47.22 & 50.95 & 51.12 & 55.11 & $\mathbf{5 1 . 3 1}$ & $\mathbf{5 2 . 3 2}$ & $\mathbf{6 0 . 1 5}$ \\
\hline
\end{tabular}

\footnotetext{
${ }^{7}$ It is important to note that clustering is an unsupervised learning method and the evaluation metrics can not be used as objective functions because the evaluation metrics requires the gold standard clustering solutions, which are not present during the clustering process.
} 
Table 7.2: F1-measure computed on 10 two view datasets

\begin{tabular}{lccccccccccc}
\hline \multirow{2}{*}{ Dataset } & \multicolumn{2}{c}{ Avg-Ensemble } & \multicolumn{2}{c}{ MOCK } & \multicolumn{3}{c}{ MMOEA } & \multicolumn{3}{c}{ MDC } \\
\cline { 2 - 11 } & v1 & v1+v2 & v1 & v1+v2 & v1 & v1+v2 & 2view & v1 & v1+v2 & 2view \\
\hline D1 & 91.28 & 91.28 & 91.54 & $\mathbf{9 1 . 6 8}$ & $\mathbf{9 1 . 9 5}$ & $\mathbf{9 1 . 6 8}$ & $\mathbf{9 2 . 0 1}$ & $\mathbf{9 1 . 9 5}$ & $\mathbf{9 1 . 6 8}$ & $\mathbf{9 2 . 0 1}$ \\
D2 & $\mathbf{9 2 . 6 1}$ & 92.61 & 92.56 & $\mathbf{9 2 . 6 9}$ & $\mathbf{9 2 . 6 1}$ & $\mathbf{9 2 . 6 9}$ & $\mathbf{9 3 . 2 8}$ & $\mathbf{9 2 . 6 1}$ & $\mathbf{9 2 . 6 9}$ & $\mathbf{9 3 . 2 8}$ \\
D3 & 88.71 & 88.71 & 89.91 & 89.91 & 90.71 & 91.74 & 92.14 & $\mathbf{9 1 . 0 3}$ & $\mathbf{9 2 . 1 1}$ & $\mathbf{9 3 . 8 2}$ \\
D4 & 84.38 & 86.45 & 86.12 & 86.93 & 86.32 & 87.23 & 89.91 & $\mathbf{8 6 . 9 9}$ & $\mathbf{8 9 . 1 8}$ & $\mathbf{9 2 . 7 8}$ \\
D5 & 66.52 & 67.56 & 67.56 & 68.32 & 69.12 & 70.34 & 71.12 & $\mathbf{7 0 . 1 2}$ & $\mathbf{7 1 . 6 1}$ & $\mathbf{7 5 . 8 2}$ \\
D6 & 65.09 & 47.97 & 66.01 & 48.13 & 67.19 & 67.73 & 70.01 & $\mathbf{6 8 . 2 1}$ & $\mathbf{7 0 . 7 2}$ & $\mathbf{7 4 . 0 5}$ \\
D7 & 56.24 & 63.67 & 58.23 & 64.39 & 62.99 & 67.16 & 71.25 & $\mathbf{6 3 . 8 7}$ & $\mathbf{6 9 . 6 3}$ & $\mathbf{7 4 . 4 2}$ \\
D8 & 67.87 & 68.01 & 70.03 & 70.97 & 74.32 & 75.46 & 76.23 & $\mathbf{7 5 . 1 2}$ & $\mathbf{7 6 . 7 4}$ & $\mathbf{7 8 . 1 3}$ \\
Citeseer & 52.87 & 56.78 & 54.01 & 58.94 & 58.87 & 59.73 & 61.23 & $\mathbf{6 1 . 1 8}$ & $\mathbf{6 1 . 7 3}$ & $\mathbf{6 8 . 2 9}$ \\
Cora & 55.54 & 56.53 & 57.98 & 58.17 & 59.62 & 61.15 & 62.21 & $\mathbf{6 0 . 5 4}$ & $\mathbf{6 2 . 1 7}$ & $\mathbf{6 3 . 5 4}$ \\
\hline
\end{tabular}

Table 7.3: Rand Index computed on 10 two view datasets

\begin{tabular}{|c|c|c|c|c|c|c|c|c|c|c|}
\hline \multirow{2}{*}{ Dataset } & \multicolumn{2}{|c|}{ Avg-Ensemble } & \multicolumn{2}{|c|}{ MOCK } & \multicolumn{3}{|c|}{ MMOEA } & \multicolumn{3}{|c|}{ MDC } \\
\hline & v1 & $\mathrm{v} 1+\mathrm{v} 2$ & v1 & $\mathrm{v} 1+\mathrm{v} 2$ & v1 & $\mathrm{v} 1+\mathrm{v} 2$ & 2view & v1 & $\mathrm{v} 1+\mathrm{v} 2$ & 2view \\
\hline D1 & 71.94 & 72.94 & 71.01 & 72.94 & 72.66 & 72.94 & 75.21 & 73.13 & 73.64 & 75.43 \\
\hline D2 & 76.14 & 76.14 & 76.99 & 76.34 & 77.89 & 78.31 & 79.89 & 78.23 & 78.51 & 80.21 \\
\hline D3 & 57.01 & 58.41 & 68.19 & 69.12 & 70.41 & 75.12 & 79.32 & 71.11 & 78.12 & 87.12 \\
\hline D4 & 59.38 & 61.55 & 69.11 & 70.33 & 74.93 & 74.93 & 75.58 & 75.45 & 78.91 & 88.21 \\
\hline D5 & 61.02 & 61.96 & 63.12 & 63.76 & 70.79 & 75.12 & 76.17 & 73.12 & 75.97 & 82.72 \\
\hline D6 & 66.73 & 48.13 & 67.53 & 50.11 & 72.65 & 73.12 & 74.65 & 74.95 & 76.32 & 80.88 \\
\hline D7 & 65.52 & 67.96 & 72.12 & 73.01 & 73.13 & 74.76 & 76.21 & 74.41 & 75.11 & 80.56 \\
\hline D8 & 75.58 & 76.83 & 75.88 & 77.63 & 80.76 & 81.12 & 82.12 & 82.92 & 83.41 & 85.14 \\
\hline Citeseer & 60.43 & 73.06 & 72.23 & 74.34 & 76.26 & 77.13 & 77.23 & 78.26 & 79.36 & 85.21 \\
\hline Cora & 70.21 & 73.92 & 76.12 & 74.23 & 78.79 & 80.21 & 81.83 & 80.11 & 81.32 & 89.75 \\
\hline
\end{tabular}


Table 7.4: Improvement of clustering quality of MDC compare to MMOEA

\begin{tabular}{lcccccccccc}
\hline & \multicolumn{3}{c}{ CA Improvement } & \multicolumn{3}{c}{ F1 Improvement } & \multicolumn{3}{c}{ RI Improvement } \\
\cline { 2 - 10 } Dataset & v1 & v1+v2 & 2view & v1 & v1+v2 & 2view & v1 & v1+v2 & 2view \\
\hline D1 & 0.000 & 0.000 & 1.525 & 0.000 & 0.000 & 0.000 & 0.647 & 0.960 & 0.293 \\
D2 & 0.000 & 0.000 & 1.139 & 0.000 & 0.000 & 0.000 & 0.437 & 0.255 & 0.401 \\
D3 & 2.520 & 1.955 & 6.238 & 0.353 & 0.403 & 1.823 & 0.994 & 3.994 & 9.834 \\
D4 & $\mathbf{3 . 0 7 5}$ & 3.668 & 4.902 & 0.776 & 2.235 & 3.192 & 0.694 & $\mathbf{5 . 3 1 2}$ & $\mathbf{1 6 . 7 1 1}$ \\
D5 & 2.936 & 3.357 & 7.273 & 1.447 & 1.806 & 6.609 & 3.291 & 1.132 & 8.599 \\
D6 & 1.507 & 2.645 & 3.552 & $\mathbf{1 . 5 1 8}$ & $\mathbf{4 . 4 1 5}$ & 5.771 & 3.166 & 4.376 & 8.346 \\
D7 & 0.194 & 0.041 & 5.069 & 1.397 & 3.678 & 4.449 & 1.750 & 0.468 & 5.708 \\
D8 & 0.000 & 0.568 & 5.006 & 1.076 & 1.696 & 2.492 & 2.675 & 2.823 & 3.678 \\
Citeseer & 2.220 & 3.635 & $\mathbf{1 0 . 6 3 7}$ & 3.924 & 3.348 & $\mathbf{1 1 . 5 3 0}$ & 2.623 & 2.891 & 10.333 \\
Cora & 0.707 & 2.347 & 9.145 & 1.543 & 1.668 & 2.138 & 1.675 & 1.384 & 9.679 \\
\hline
\end{tabular}

\subsubsection{Comparison on Two View Datasets}

Our method MDC was compared with three clustering methods: AVGEnsemble, MOCK and MMOEA. The first method is a single objective average-link clustering ensemble method based on link pairwise similarity matrices [72]. The second method MOCK is a multi-objective evolutionary algorithm [62] based on SPEA- $\mathrm{II}^{8}$. Our recent work, MMOEA is a multi-objective multi-view evolutionary algorithm based on the NSGAII approach [132]. MMOEA uses the standard crossover method with three objectives. We implemented Avg-Ensemble and MMOEA clustering methods and the code for MOCK was provided by their authors.

Tables 7.1, 7.2 and 7.3 show the values of CA, F1 and RI respectively computed on 10 different two-view datasets. The column label "v1" means view one and "v1+v2" means that view one and view two were concatenated in a single feature matrix. "2view" means the two views v1 and v2 were used separately and two feature matrices were constructed in the clustering process. The bold values indicate the algorithm producing the highest value for the given dataset in a specific view. All three tables show

\footnotetext{
${ }^{8} \mathrm{http}: / /$ personalpages.manchester.ac.uk/mbs/julia.handl/mock.html
} 
a general trend that our new method, MDC, outperforms other clustering methods in terms of CA, F1 and RI on all views.

Table 7.4 provides the percentage improvements of MDC over MMOEA. The percentage values are calculated in terms of CA, F1 and RI on 10 different datasets. In general, MDC performs equally well for a few cases, but most of the time shows a reasonable improvement over MMOEA.

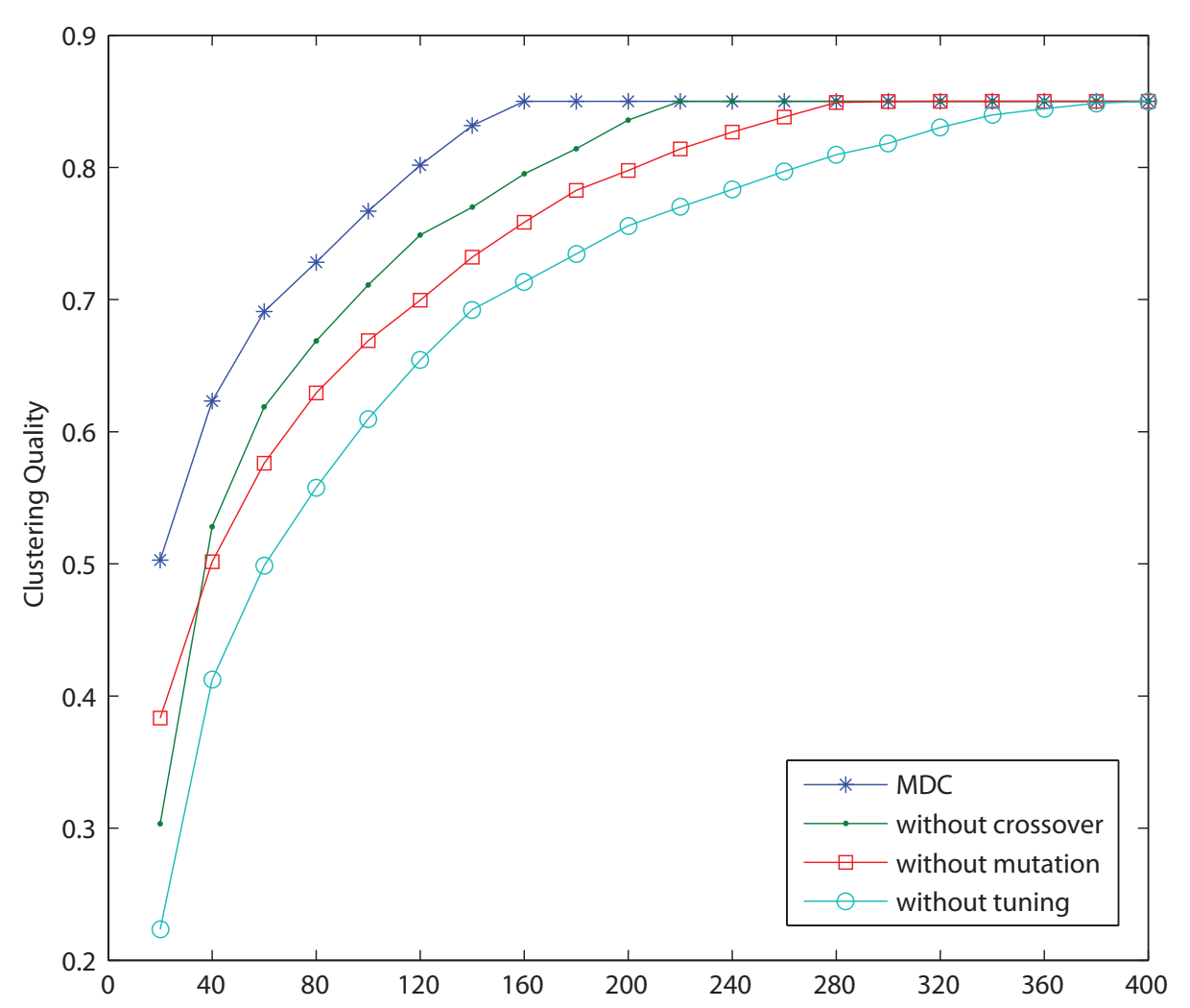

Figure 7.5: Analysis of MDC

We further performed component analysis of MDC by removing different evolutionary steps and analyzing the results on two view dataset. Figure 7.5 shows the clustering quality (Average $\mathrm{CA}$ ) on y-axis and number of generations on $\mathrm{x}$-axis. The results were computed by removing crossover, mutation and tuning steps individually from the MDC method and producing the clustering solution. With all features, MDC converged around 
160 generations. However, when the tuning step was removed the method converged around 375 generations. The tuning step played a vital role in improving the convergence speed. Crossover and mutation steps also contributed to the speed but less than tuning step.

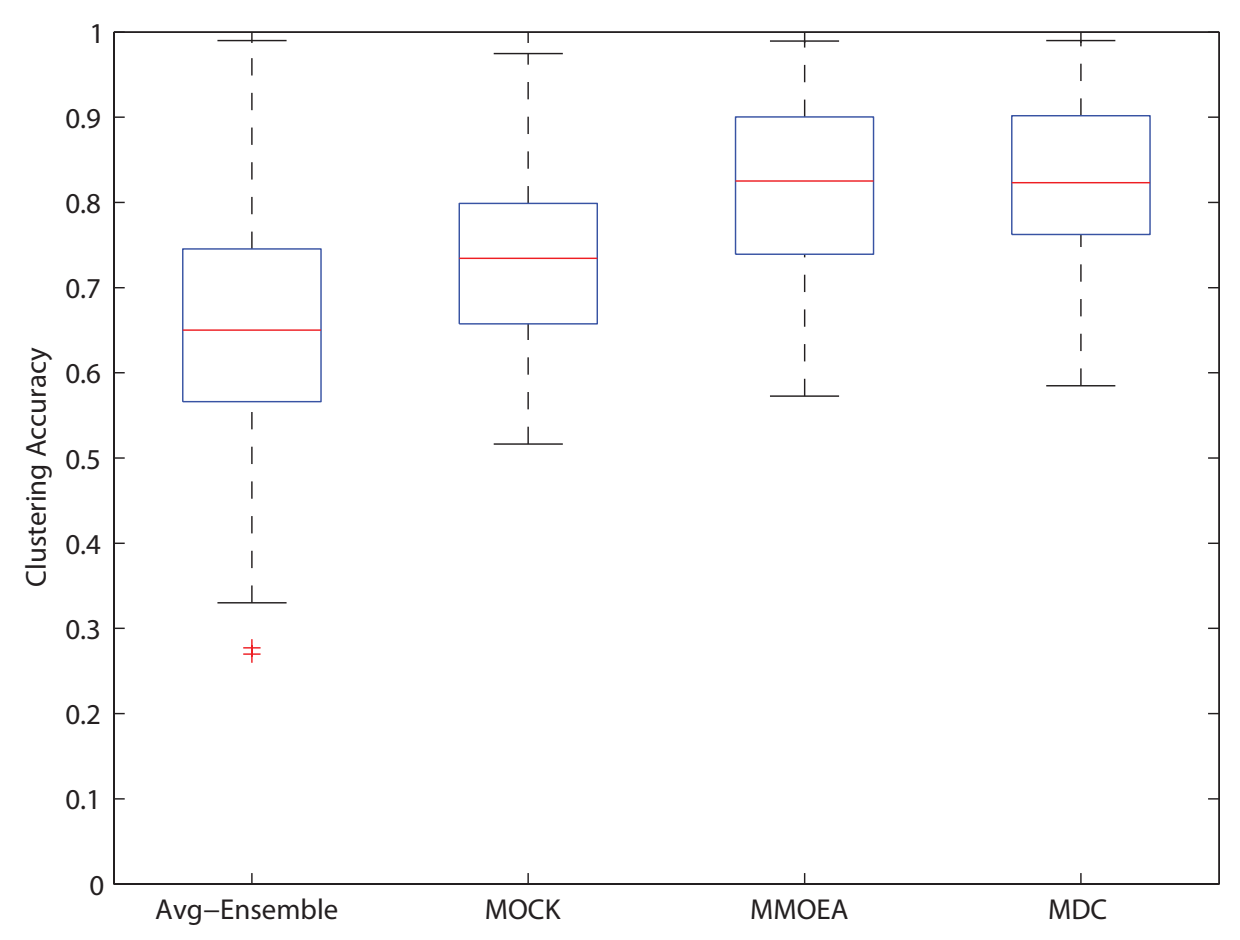

Figure 7.6: CA on combined dataset

\subsubsection{Comparison on Three View Datasets}

The results of the three view / combined dataset of AMBIENT, MORESQUE and ODP-239 are depicted in Figure 7.6, 7.7 and 7.8 as box plots of CA, F1 and RI values. The three views were concatenated for Avg-Ensemble and MOCK and used separately for MMOEA and MDC. Our method MDC has better mean values as compared to Avg-Ensemble, MOCK and MMOEA in terms of CA, F1 and RI values. Similar results were also observed on other views. 


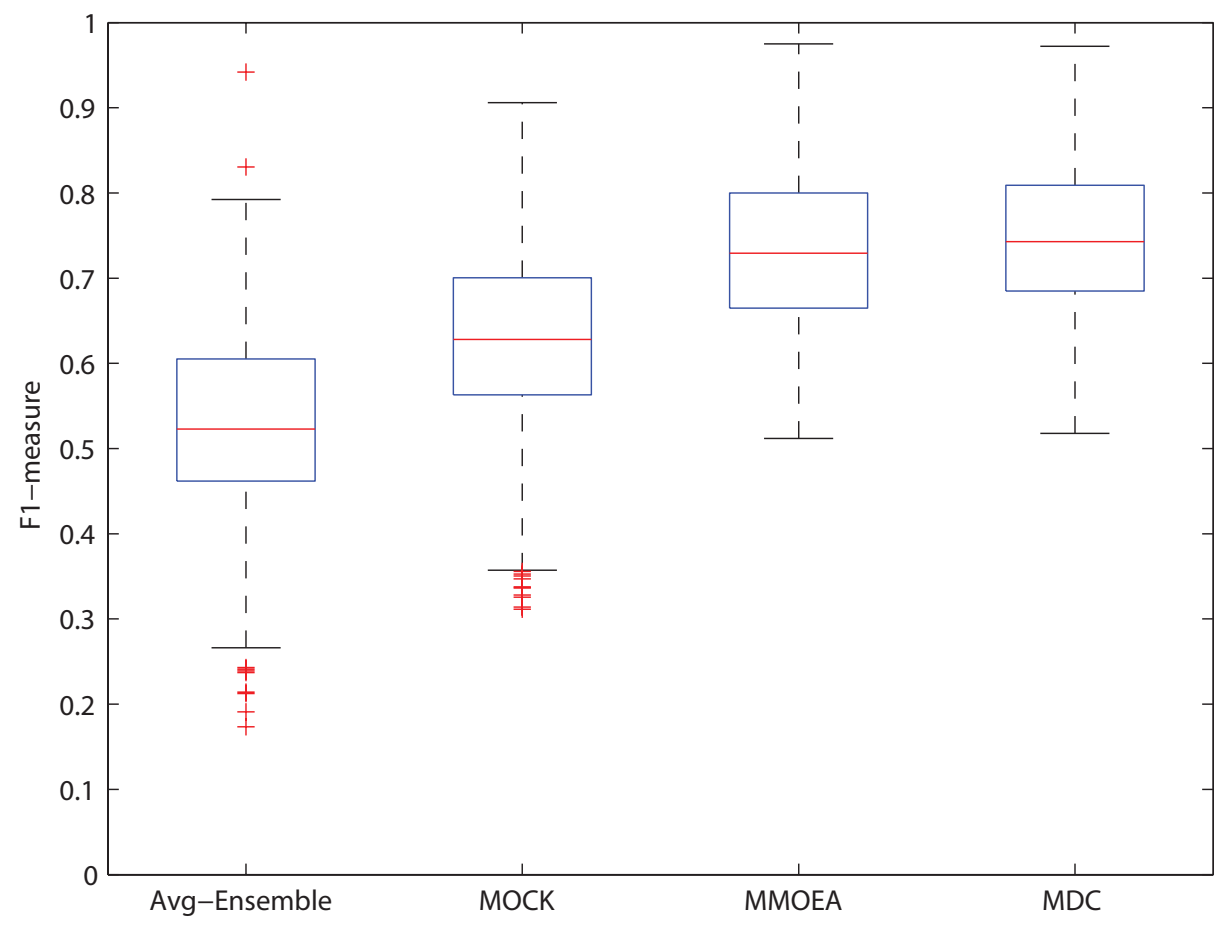

Figure 7.7: F1 on combined dataset

\subsubsection{Statistical Analysis}

We performed the pairwise Wilcoxon statistical significance test [138] on two view and three view datasets for CA, F1 and RI values. We used CA, F1 and RI values of MDC as a control group and compared them individually with the values of Avg-Ensemble, Mock and MMOEA. Table 7.5 shows the p-values of the statistical test performed on CA, F1 and RI values of two view datasets(D1-D8, Citeseer and Cora).

The p-values of the statistical test performed on CA, F1 and RI values of combined dataset (having 397 queries) are 0.0016, 0.0002 and 0.0001 respectively. We used $\alpha=0.05$ for all statistical test and the results showed that our method MDC has a statistically significant improvement as compared to other clustering methods. 


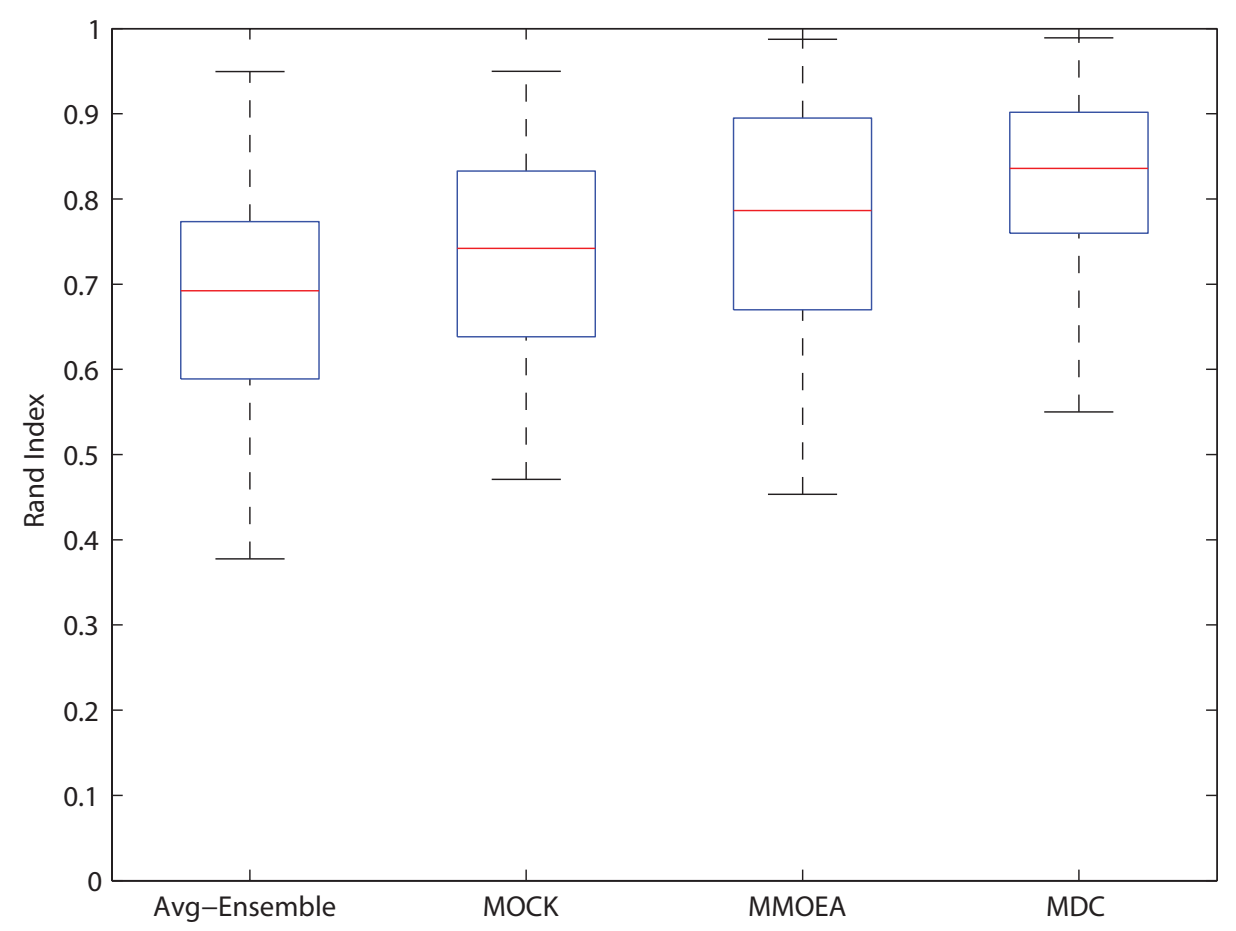

Figure 7.8: RI on combined dataset

\subsection{Experimental Setup for MOMVEC}

As chapter 6 showed MMOEA was consistently better than other clustering methods and there is no point in listing all the methods. Therefore we compared MMOEA, MDC and MOMVEC methods in this experiment.

MOMVEC is similar to MDC except for the fact that MOMVEC uses the NSGA-II approach to compute the Pareto fronts and differs in terms of implementation of the algorithm.

MOMVEC uses the following parameter settings: maximum number of generations $=1000$, crossover probability $\frac{1}{6}$, mutation probability $\frac{1}{3}$, population size $=20$. We used a fixed number of clusters (provided by user) for all other algorithms to generate initial candidate clustering solutions. MOMVEC was compared with MMOEA and MDC using three different metrics: Clustering Accuracy (CA) [105], F1-measure (F1) [30], 
Table 7.5: Statistical significance test based on the values of Clustering Accuracy, F1-measure and Rand Index computed on all datasets

\begin{tabular}{lcccccccc}
\hline & \multicolumn{2}{c}{ Avg-Ensemble } & \multicolumn{2}{c}{ MOCK } & \multicolumn{3}{c}{ MMOEA } \\
\cline { 2 - 8 } & $\mathrm{v} 1$ & $\mathrm{v} 1+\mathrm{v} 2$ & $\mathrm{v} 1$ & $\mathrm{v} 1+\mathrm{v} 2$ & $\mathrm{v} 1$ & $\mathrm{v} 1+\mathrm{v} 2$ & $2 \mathrm{view}$ \\
\hline p-value on CA & 0.0015 & 0.0008 & 0.0025 & 0.0009 & 0.0154 & 0.0057 & 0.0001 \\
p-value on F1 & 0.0017 & 0.0185 & 0.0056 & 0.0447 & 0.0042 & 0.0026 & 0.0038 \\
p-value on RI & 0.0003 & 0.003 & 0.0004 & 0.008 & 0.0005 & 0.0021 & 0.0011 \\
\hline
\end{tabular}

and Rand Index (RI) [70], and on 10 two-view datasets (D1-D8, Citeseer and Cora), and one three-view dataset (combined version of AMBIENT, MORESQUE and ODP-239).

Table 7.6: Values of CA computed on 10 two-view datasets

\begin{tabular}{lcccccccccc}
\hline \multirow{2}{*}{ Dataset } & \multicolumn{3}{c}{ MMOEA } & \multicolumn{3}{c}{ MDC } & \multicolumn{3}{c}{ MOMVEC } \\
\cline { 2 - 9 } & $\mathrm{v} 1$ & $\mathrm{v} 1+\mathrm{v} 2$ & 2 view & v1 & v1+v2 & 2view & v1 & v1+v2 & 2view \\
\hline D1 & $\mathbf{9 1 . 1 2}$ & $\mathbf{9 1 . 1 2}$ & 91.12 & $\mathbf{9 1 . 1 2}$ & $\mathbf{9 1 . 1 2}$ & $\mathbf{9 2 . 5 1}$ & $\mathbf{9 1 . 1 2}$ & $\mathbf{9 1 . 1 2}$ & $\mathbf{9 2 . 5 1}$ \\
D2 & 90.12 & $\mathbf{9 0 . 2 1}$ & 90.42 & 90.12 & $\mathbf{9 0 . 2 1}$ & $\mathbf{9 1 . 4 5}$ & $\mathbf{9 0 . 1 5}$ & $\mathbf{9 0 . 2 1}$ & $\mathbf{9 1 . 4 5}$ \\
D3 & 84.91 & 86.43 & 86.72 & 87.05 & 88.12 & $\mathbf{9 2 . 1 3}$ & $\mathbf{8 7 . 1 1}$ & $\mathbf{8 8 . 1 9}$ & $\mathbf{9 2 . 1 3}$ \\
D4 & 85.54 & 86.96 & 88.54 & 88.17 & 90.15 & $\mathbf{9 2 . 8 8}$ & $\mathbf{8 8 . 1 8}$ & $\mathbf{9 0 . 1 8}$ & $\mathbf{9 2 . 8 8}$ \\
D5 & 68.12 & 68.82 & 70.12 & 70.12 & $\mathbf{7 1 . 1 3}$ & 75.22 & $\mathbf{7 0 . 1 3}$ & $\mathbf{7 1 . 1 1}$ & $\mathbf{7 5 . 2 3}$ \\
D6 & 73.01 & 74.87 & 75.45 & 74.11 & 76.85 & $\mathbf{7 8 . 1 3}$ & $\mathbf{7 4 . 1 2}$ & $\mathbf{7 6 . 8 7}$ & $\mathbf{7 8 . 1 3}$ \\
D7 & 72.17 & 72.51 & 74.17 & 72.31 & $\mathbf{7 2 . 5 4}$ & $\mathbf{7 7 . 9 3}$ & $\mathbf{7 2 . 3 4}$ & $\mathbf{7 2 . 5 4}$ & $\mathbf{7 7 . 9 3}$ \\
D8 & $\mathbf{7 9 . 9 1}$ & 80.98 & 79.91 & $\mathbf{7 9 . 9 1}$ & 81.44 & $\mathbf{8 3 . 9 1}$ & $\mathbf{7 9 . 9 1}$ & $\mathbf{8 1 . 5 4}$ & $\mathbf{8 3 . 9 1}$ \\
Citeseer & 50.01 & 51.45 & 54.34 & $\mathbf{5 1 . 1 2}$ & $\mathbf{5 3 . 3 2}$ & $\mathbf{6 0 . 1 2}$ & $\mathbf{5 1 . 1 2}$ & $\mathbf{5 3 . 3 2}$ & $\mathbf{6 0 . 1 2}$ \\
Cora & 50.95 & 51.12 & 55.11 & $\mathbf{5 1 . 3 1}$ & $\mathbf{5 2 . 3 2}$ & $\mathbf{6 0 . 1 5}$ & $\mathbf{5 1 . 3 1}$ & $\mathbf{5 2 . 3 2}$ & $\mathbf{6 0 . 1 5}$ \\
\hline
\end{tabular}

\subsubsection{Comparison on Two View Datasets}

Tables 7.6, 7.7 and 7.8 present the average values of 100 runs of the clustering methods in terms of CA, F1 and RI values respectively ${ }^{9}$. These values

\footnotetext{
${ }^{9}$ values are converted to percentages by multiplying them with 100 for better understanding
} 
Table 7.7: Values of F1-measure computed on 10 two-view datasets

\begin{tabular}{lcccccccccc}
\hline & \multicolumn{3}{c}{ MMOEA } & \multicolumn{3}{c}{ MDC } & \multicolumn{3}{c}{ MOMVEC } \\
\cline { 2 - 9 } Dataset & $\mathrm{v} 1$ & $\mathrm{v} 1+\mathrm{v} 2$ & 2view & $\mathrm{v} 1$ & $\mathrm{v} 1+\mathrm{v} 2$ & 2view & $\mathrm{v} 1$ & $\mathrm{v} 1+\mathrm{v} 2$ & 2view \\
\hline D1 & $\mathbf{9 1 . 9 5}$ & $\mathbf{9 1 . 6 8}$ & $\mathbf{9 2 . 0 1}$ & $\mathbf{9 1 . 9 5}$ & $\mathbf{9 1 . 6 8}$ & $\mathbf{9 2 . 0 1}$ & $\mathbf{9 1 . 9 5}$ & $\mathbf{9 1 . 6 8}$ & $\mathbf{9 2 . 0 1}$ \\
D2 & $\mathbf{9 2 . 6 1}$ & $\mathbf{9 2 . 6 9}$ & $\mathbf{9 3 . 2 8}$ & $\mathbf{9 2 . 6 1}$ & $\mathbf{9 2 . 6 9}$ & $\mathbf{9 3 . 2 8}$ & $\mathbf{9 2 . 6 1}$ & $\mathbf{9 2 . 6 9}$ & $\mathbf{9 3 . 2 8}$ \\
D3 & 90.71 & 91.74 & 92.14 & 91.03 & 92.11 & 93.82 & $\mathbf{9 1 . 1 1}$ & $\mathbf{9 2 . 1 4}$ & $\mathbf{9 3 . 8 3}$ \\
D4 & 86.32 & 87.23 & 89.91 & 86.99 & $\mathbf{8 9 . 1 8}$ & 92.78 & $\mathbf{8 7 . 1 2}$ & 89.12 & $\mathbf{9 2 . 7 9}$ \\
D5 & 69.12 & 70.34 & 71.12 & 70.12 & 71.61 & $\mathbf{7 5 . 8 2}$ & $\mathbf{7 0 . 2 2}$ & $\mathbf{7 1 . 6 3}$ & 75.81 \\
D6 & 67.19 & 67.73 & 70.01 & 68.21 & 70.72 & 74.05 & $\mathbf{6 8 . 3 1}$ & $\mathbf{7 0 . 7 3}$ & $\mathbf{7 4 . 0 7}$ \\
D7 & 62.99 & 67.16 & 71.25 & $\mathbf{6 3 . 8 7}$ & 69.63 & $\mathbf{7 4 . 4 2}$ & $\mathbf{6 3 . 8 7}$ & $\mathbf{6 9 . 6 5}$ & 74.41 \\
D8 & 74.32 & 75.46 & 76.23 & 75.12 & 76.74 & 78.13 & $\mathbf{7 5 . 1 5}$ & $\mathbf{7 6 . 7 5}$ & $\mathbf{7 8 . 1 4}$ \\
Citeseer & 58.87 & 59.73 & 61.23 & $\mathbf{6 1 . 1 8}$ & $\mathbf{6 1 . 7 3}$ & $\mathbf{6 8 . 2 9}$ & $\mathbf{6 1 . 1 8}$ & $\mathbf{6 1 . 7 3}$ & $\mathbf{6 8 . 2 9}$ \\
Cora & 59.62 & 61.15 & 62.21 & $\mathbf{6 0 . 5 4}$ & $\mathbf{6 2 . 1 7}$ & $\mathbf{6 3 . 5 4}$ & $\mathbf{6 0 . 5 4}$ & $\mathbf{6 2 . 1 7}$ & $\mathbf{6 3 . 5 4}$ \\
\hline
\end{tabular}

Table 7.8: Values of Rand Index computed on 10 two-view datasets

\begin{tabular}{lcccccccccc}
\hline & \multicolumn{3}{c}{ MMOEA } & \multicolumn{3}{c}{ MDC } & \multicolumn{3}{c}{ MOMVEC } \\
\cline { 2 - 10 } Dataset & v1 & v1+v2 & 2view & v1 & v1+v2 & 2view & v1 & v1+v2 & 2view \\
\hline D1 & 72.66 & 72.94 & 75.21 & $\mathbf{7 3 . 1 3}$ & $\mathbf{7 3 . 6 4}$ & $\mathbf{7 5 . 4 3}$ & $\mathbf{7 3 . 1 3}$ & $\mathbf{7 3 . 6 4}$ & $\mathbf{7 5 . 4 3}$ \\
D2 & 77.89 & 78.31 & 79.89 & $\mathbf{7 8 . 2 3}$ & $\mathbf{7 8 . 5 1}$ & $\mathbf{8 0 . 2 1}$ & $\mathbf{7 8 . 2 3}$ & $\mathbf{7 8 . 5 1}$ & $\mathbf{8 0 . 2 1}$ \\
D3 & 70.41 & 75.12 & 79.32 & 71.11 & $\mathbf{7 8 . 1 2}$ & $\mathbf{8 7 . 1 2}$ & $\mathbf{7 1 . 1 2}$ & $\mathbf{7 8 . 1 2}$ & $\mathbf{8 7 . 1 2}$ \\
D4 & 74.93 & 74.93 & 75.58 & $\mathbf{7 5 . 4 5}$ & 78.91 & 88.21 & $\mathbf{7 5 . 4 5}$ & $\mathbf{7 8 . 9 3}$ & $\mathbf{8 8 . 3 2}$ \\
D5 & 70.79 & 75.12 & 76.17 & $\mathbf{7 3 . 1 2}$ & 75.97 & $\mathbf{8 2 . 7 2}$ & $\mathbf{7 3 . 1 2}$ & $\mathbf{7 6 . 0 7}$ & $\mathbf{8 2 . 7 2}$ \\
D6 & 72.65 & 73.12 & 74.65 & $\mathbf{7 4 . 9 5}$ & 76.32 & 80.88 & $\mathbf{7 4 . 9 5}$ & $\mathbf{7 6 . 4 1}$ & $\mathbf{8 0 . 9 1}$ \\
D7 & 73.13 & 74.76 & 76.21 & 74.41 & $\mathbf{7 5 . 1 1}$ & $\mathbf{8 0 . 5 6}$ & $\mathbf{7 4 . 4 3}$ & $\mathbf{7 5 . 1 1}$ & $\mathbf{8 0 . 5 6}$ \\
D8 & 80.76 & 81.12 & 82.12 & $\mathbf{8 2 . 9 2}$ & 83.41 & $\mathbf{8 5 . 1 4}$ & $\mathbf{8 2 . 9 4}$ & $\mathbf{8 3 . 4 5}$ & $\mathbf{8 5 . 1 4}$ \\
Citeseer & 76.26 & 77.13 & 77.23 & $\mathbf{7 8 . 2 6}$ & $\mathbf{7 9 . 3 6}$ & $\mathbf{8 5 . 2 1}$ & $\mathbf{7 8 . 2 6}$ & $\mathbf{7 9 . 3 6}$ & $\mathbf{8 5 . 2 1}$ \\
Cora & 78.79 & 80.21 & 81.83 & $\mathbf{8 0 . 1 1}$ & $\mathbf{8 1 . 3 2}$ & $\mathbf{8 9 . 7 5}$ & $\mathbf{8 0 . 1 1}$ & $\mathbf{8 1 . 3 2}$ & $\mathbf{8 9 . 7 5}$ \\
\hline
\end{tabular}


Table 7.9: Percentage improvement of MOMVEC compared to MDC

\begin{tabular}{lccccccccccc}
\hline \multirow{2}{*}{ Dataset } & \multicolumn{3}{c}{ CA Improvement } & \multicolumn{3}{c}{ F1 Improvement } & \multicolumn{3}{c}{ RI Improvement } \\
\cline { 2 - 10 } & $\mathrm{v} 1$ & $\mathrm{v} 1+\mathrm{v} 2$ & $2 \mathrm{view}$ & $\mathrm{v} 1$ & $\mathrm{v} 1+\mathrm{v} 2$ & $2 \mathrm{view}$ & $\mathrm{v} 1$ & $\mathrm{v1}+\mathrm{v} 2$ & $2 \mathrm{view}$ \\
\hline D1 & 0.000 & 0.000 & 0.000 & 0.000 & 0.000 & 0.000 & 0.000 & 0.000 & 0.000 \\
D2 & 0.000 & 0.000 & 0.000 & $\mathbf{0 . 0 3 3}$ & 0.000 & 0.000 & 0.000 & 0.000 & 0.000 \\
D3 & $\mathbf{0 . 0 8 8}$ & $\mathbf{0 . 0 3 3}$ & $\mathbf{0 . 0 1 1}$ & $\mathbf{0 . 0 6 9}$ & $\mathbf{0 . 0 7 9}$ & 0.000 & $\mathbf{0 . 0 1 4}$ & 0.000 & 0.000 \\
D4 & $\mathbf{0 . 1 4 9}$ & -0.067 & $\mathbf{0 . 0 1 1}$ & $\mathbf{0 . 0 1 1}$ & $\mathbf{0 . 0 3 3}$ & 0.000 & 0.000 & $\mathbf{0 . 0 2 5}$ & $\mathbf{0 . 1 2 5}$ \\
D5 & $\mathbf{0 . 1 4 3}$ & $\mathbf{0 . 0 2 8}$ & -0.013 & $\mathbf{0 . 0 1 4}$ & -0.028 & $\mathbf{0 . 0 1 3}$ & 0.000 & $\mathbf{0 . 1 3 2}$ & 0.000 \\
D6 & $\mathbf{0 . 1 4 7}$ & $\mathbf{0 . 0 1 4}$ & $\mathbf{0 . 0 2 7}$ & $\mathbf{0 . 0 1 3}$ & $\mathbf{0 . 0 2 6}$ & 0.000 & 0.000 & $\mathbf{0 . 1 1 8}$ & $\mathbf{0 . 0 3 7}$ \\
D7 & 0.000 & $\mathbf{0 . 0 2 9}$ & -0.013 & $\mathbf{0 . 0 4 1}$ & 0.000 & 0.000 & $\mathbf{0 . 0 2 7}$ & 0.000 & 0.000 \\
D8 & $\mathbf{0 . 0 4 0}$ & $\mathbf{0 . 0 1 3}$ & $\mathbf{0 . 0 1 3}$ & 0.000 & $\mathbf{0 . 1 2 3}$ & 0.000 & $\mathbf{0 . 0 2 4}$ & $\mathbf{0 . 0 4 8}$ & 0.000 \\
Citeseer & 0.000 & 0.000 & 0.000 & 0.000 & 0.000 & 0.000 & 0.000 & 0.000 & 0.000 \\
Cora & 0.000 & 0.000 & 0.000 & 0.000 & 0.000 & 0.000 & 0.000 & 0.000 & 0.000 \\
\hline
\end{tabular}

are computed on two view datasets specified in the first column for their corresponding clustering methods. The v1 and v1+v2 columns for all clustering methods indicate that the value is computed for view one (terms) and a concatenation of two views (view one and view two) into a single feature matrix. The 2view column mentioned under MMOEA, MDC and MOMVEC indicates that the view one and view two were each used in the clustering methods separately with two different feature matrices.

The best CA, F1 and RI values for v1, v1+v2 and 2view are shown in bold font. Overall, MOMVEC performed at least equally well to the other clustering methods in terms of CA, F1 and RI and generally out-performed the other clustering methods.

In table 7.6, MMOEA, MDC and MOMVEC share the maximum value of $\mathbf{7 9 . 9 1}$ for view one on the D8 dataset. However, MOMVEC outperformed all other clustering methods in the $\mathrm{v} 1+\mathrm{v} 2$ view.

In table 7.7, MMOEA, MDC and MOMVEC share the highest F1 values on the D1 and D2 datasets for all views. However, for the rest of the datasets, MMOEA produced worse results than both MDC and MOMVEC. Generally, MOMVEC performed slightly better than MDC clustering method. 
Table 7.10: Statistical significance test based on the values of Clustering Accuracy, F1-measure and Rand Index computed on all datasets

\begin{tabular}{lcccc|cccc}
\hline & \multicolumn{2}{c}{ Avg-Ensemble } & \multicolumn{2}{c}{ MOCK } & \multicolumn{3}{c}{ MMOEA } \\
\cline { 2 - 8 } & $\mathrm{v} 1$ & $\mathrm{v} 1+\mathrm{v} 2$ & $\mathrm{v} 1$ & $\mathrm{v} 1+\mathrm{v} 2$ & $\mathrm{v} 1$ & $\mathrm{v} 1+\mathrm{v} 2$ & 2view \\
\hline p-value on CA & 0.0015 & 0.0008 & 0.0025 & 0.0015 & 0.0147 & 0.0052 & 0.0001 \\
p-value on F1 & 0.0015 & 0.0185 & 0.0049 & 0.0447 & 0.0031 & 0.0025 & 0.0038 \\
p-value on RI & 0.0003 & 0.003 & 0.0004 & 0.0081 & 0.0005 & 0.002 & 0.0011 \\
\hline
\end{tabular}

Table 7.8 shows that MDC and MOMVEC produced very similar results, and outperformed MMOEA on all datasets in terms of RI on all views.

Table 7.9 shows the percentage improvements of MOMVEC over MDC in terms of CA, F1 and RI values on datasets having two views. The highlighted values represent the positive improvement achieved by the MOMVEC algorithm. The four negative value show the percentage cases where MOMVEC results were worse.

MOMVEC was never worse than MDC in terms of RI. Overall, MOMVEC shows similar results with MDC with a few slight improvements over MDC.

Figure 7.9 shows the performance of MOMVEC when we independently took out crossover, mutation and tuning steps. The y-axis represents the average Clustering Quality (Average CA) computed on two view datasets and the $x$-axis represents the number of generations. The algorithm converged after 240 generations with all steps, 600 generations without the tuning step, 455 generations without the mutation step and 360 generations without the crossover step. This analysis provided insight about the importance and impact of each step. As with MDC the tuning step (i.e. local search) had the greatest impact on the speed of convergence of the algorithm. The mutation methods were more important for speed of convergence (though not for early accuracy) than the crossover methods. 


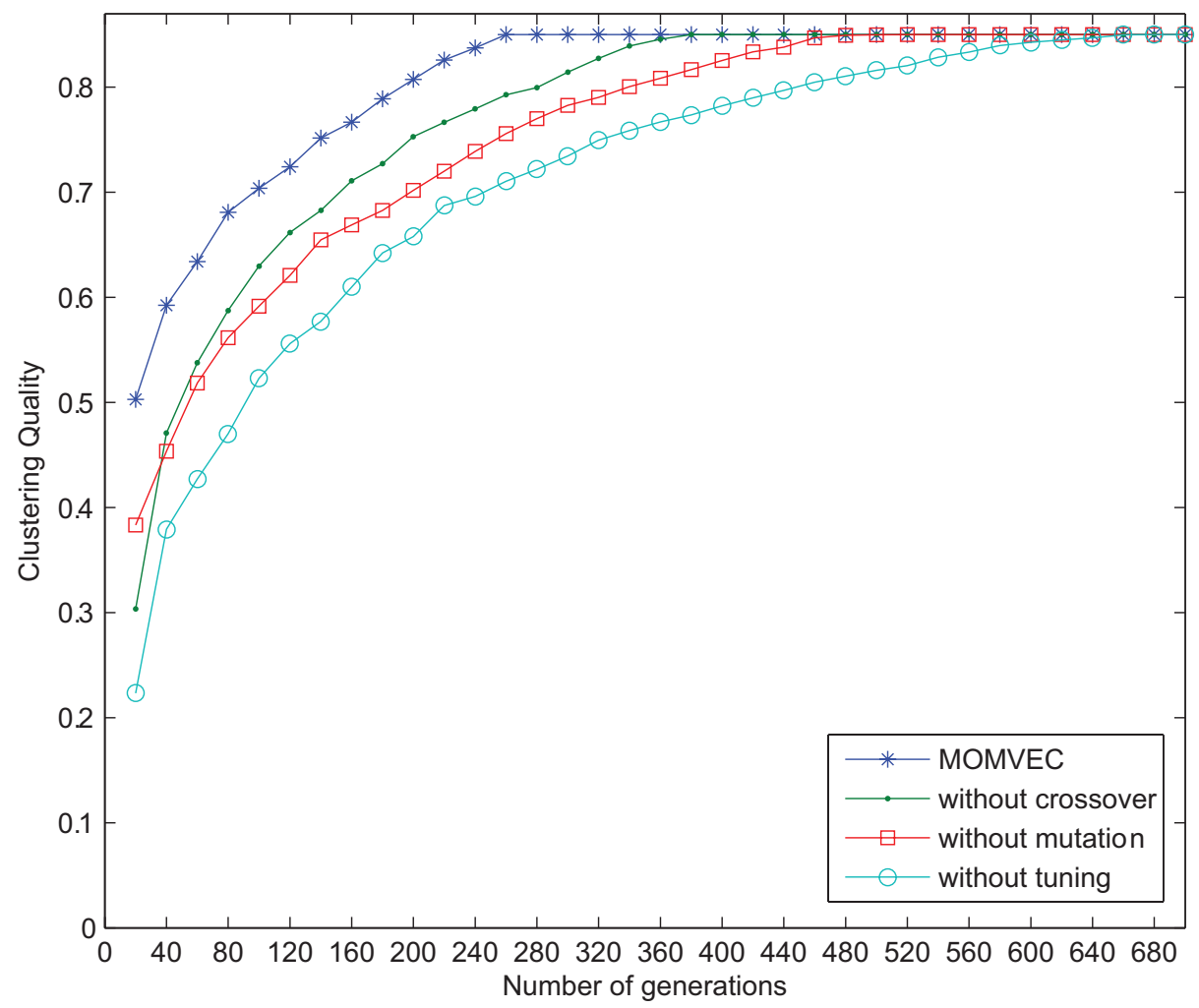

Figure 7.9: Comparison of average clustering quality (CA) of MOMVEC, MOMVEC without tuning, MOMVEC without mutation, MOMVEC without crossover steps for 700 generations. 


\subsubsection{Comparison on Three View Datasets}

We also computed CA, F1 and RI values on the combined dataset of 397 queries for all views ${ }^{10}$ which also showed that MDC and MOMVEC outperformed other clustering methods. Figures 7.10,7.11 and 7.12 shows CA, F1 and RI values computed on combined dataset respectively. MDC and MOMVEC performed equally well in terms of CA, F1 and RI values and produced better results than Avg-Ensemble, Mock and MMOEA clustering methods.

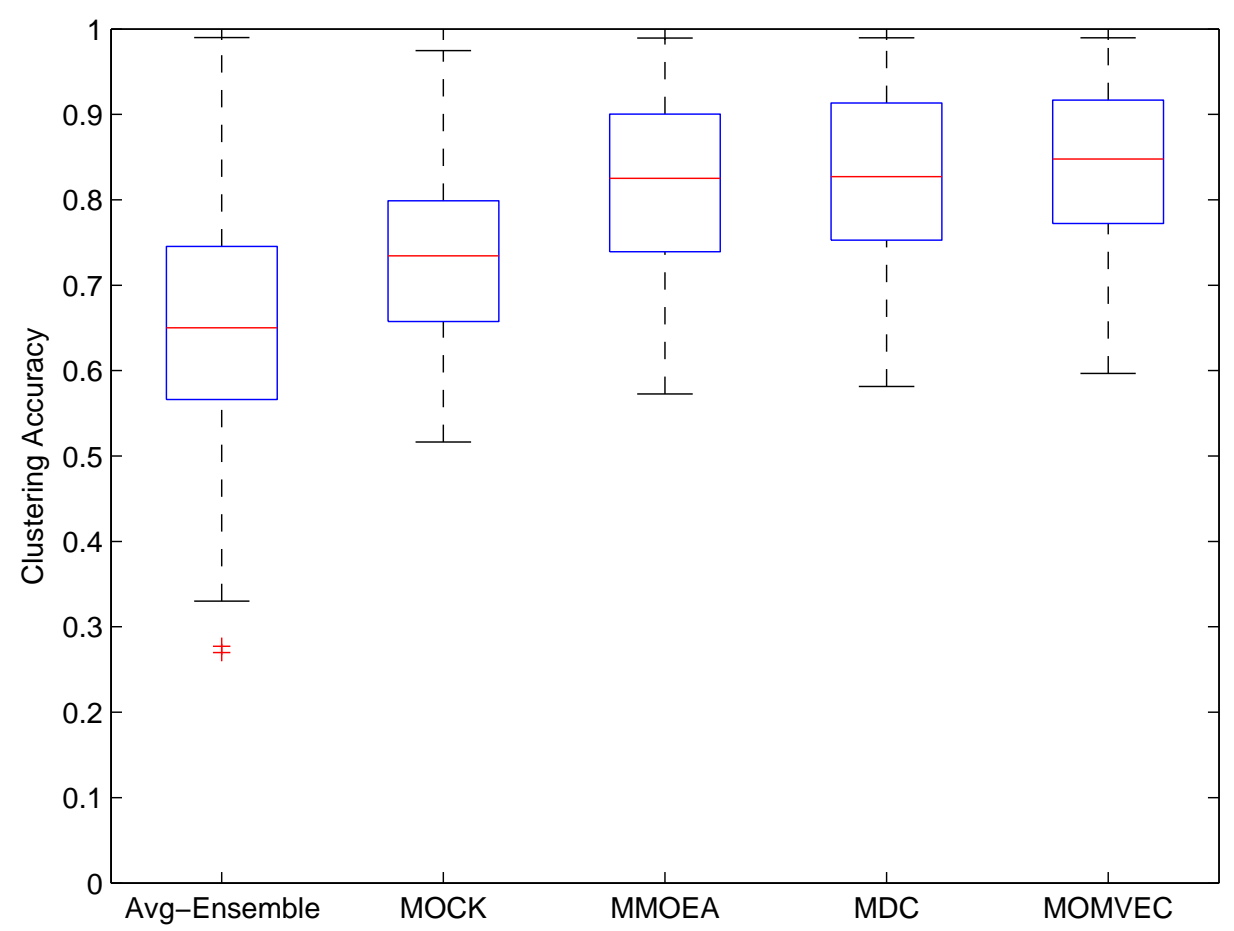

Figure 7.10: box plot of Clustering Accuracy on combined dataset

\footnotetext{
${ }^{10}$ we concatenated three views and formed one feature matrix for Avg-Ensemble and MOCK and generated three feature matrices from views separately for MMOEA, MDC and MOMVEC for fair comparison
} 


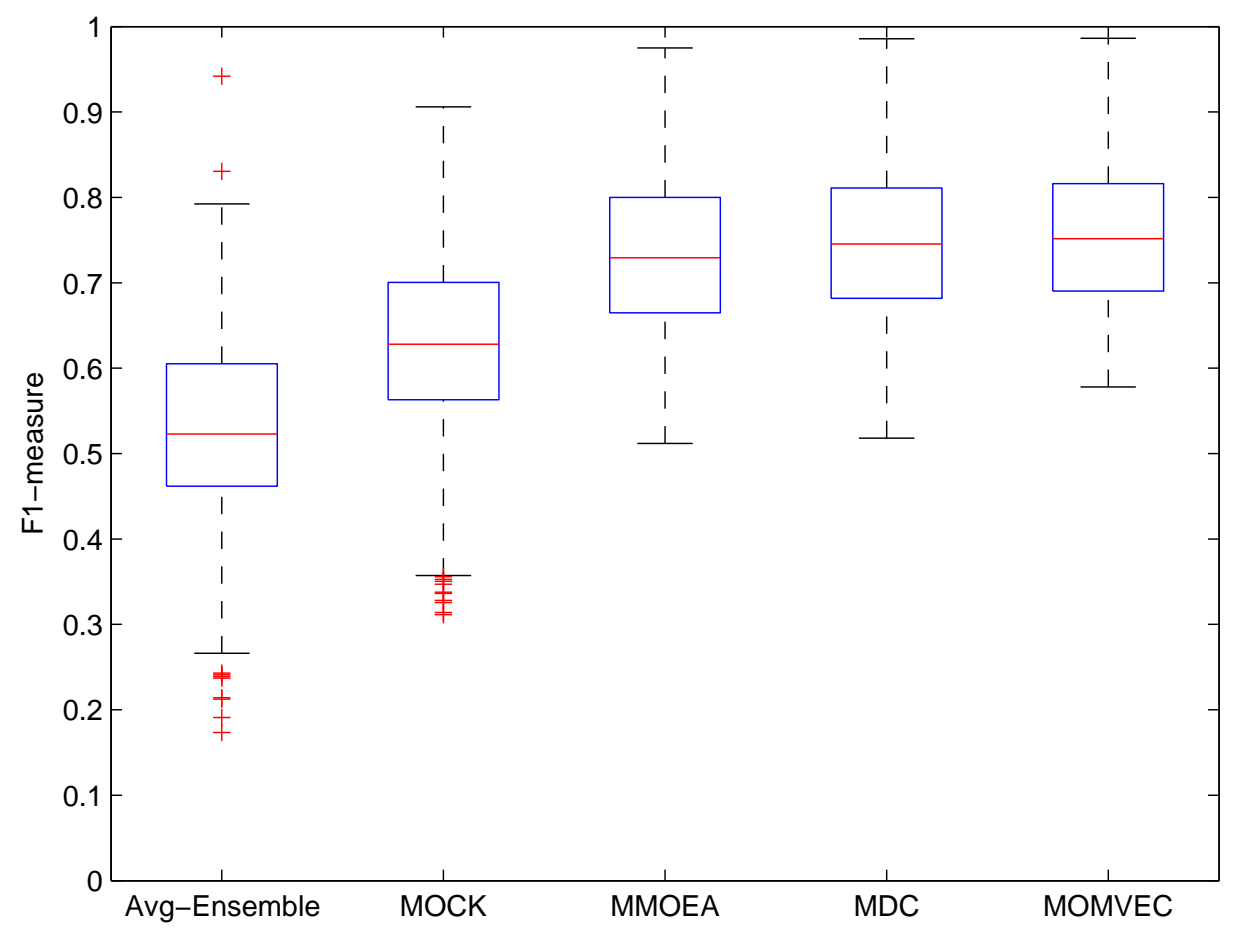

Figure 7.11: box plot of F1-measure values on combined dataset

\subsubsection{Statistical Analysis}

We performed the pairwise Wilcoxon statistical significance test [138] on CA, F1 and RI values computed on both two view and three view datasets. The values for MOMVEC were treated as a control group and were compared individually with the values of the other clustering methods.

MDC and MOMVEC performed very similarly on two view datasets and there was no statistically significance improvement. However, MOMVEC showed a statistically significant improvement compared to Avg-Ensemble, Mock and MMOEA clustering methods

Table 7.10 provides the detail of the $p$-values of 2 view datasets. The p-values for the two-view datasets calculated on Clustering Accuracy, F1measure and RI values are less than $\mathbf{0 . 0 0 5}$. The statistical test was performed for $\alpha=\mathbf{0 . 0 5}$ and the results showed that the improvement of MOMVEC 


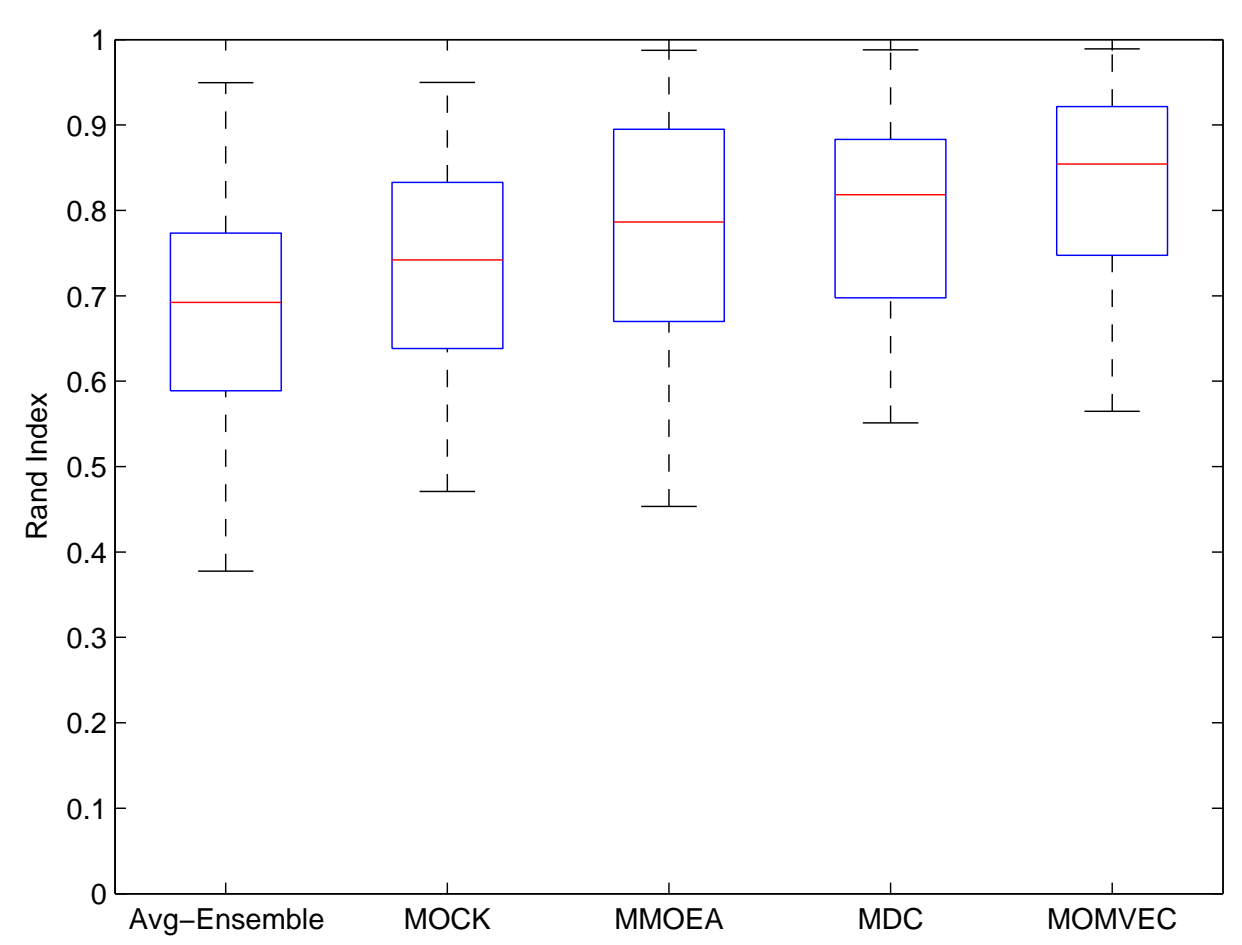

Figure 7.12: box plot of RI values on combined dataset

on Avg-Ensemble, Mock and MMOEA were statistically significant.

The $\mathrm{p}$-values for the three view datasets calculated on Clustering Accuracy, F1-measure and RI showed the similar trend as two view datasets. The p-values are less than $\mathbf{0 . 0 0 5}$ for $\alpha=\mathbf{0 . 0 5}$ in case of Avg-Ensemble, Mock and MMOEA but the p-values are greater than $\mathbf{0 . 0 0 5}$ in case of MDC on three view datasets. This means MOMVEC showed statistically significant performance as compared to Avg-Ensemble, Mock and MMOEA except for MDC.

\subsection{Single vs Multi-Objective Analysis}

We also implemented two single objective methods GA-1 and GA-2. The GA-1 uses $O b j_{1}$ and GA-2 uses $O b j_{2}$ only. Table 7.11 shows F1 score computed on two view datasets for GA-1, GA-2, MDC and MOMVEC for com- 
parison. MDC outperformed single objective clustering methods GA-1, GA-2. This analysis was performed to see if generating diverse clustering is the only reason for the better performance. However, the result show that using multi-objective approach can improve the results.

Table 7.11: Comparison of F1 score of GA-1, GA-2, MDC (SPEA-II) and MOMVEC (NSGA-II) computed on 10 different multi-view datasets

\begin{tabular}{lccccccccccc}
\hline \multirow{2}{*}{ Dataset } & \multicolumn{3}{c}{ GA-1 } & \multicolumn{2}{c}{ GA-2 } & \multicolumn{3}{c}{ MDC (SPEA-II) } & \multicolumn{3}{c}{ MOMVEC (NSGA-II) } \\
\cline { 2 - 11 } & v1 & v1+v2 & v1 & v1+v2 & v1 & v1+v2 & 2view & v1 & v1+v2 & 2view \\
\hline D1 & 90.18 & 91.28 & 81.88 & 82.28 & $\mathbf{9 1 . 9 5}$ & $\mathbf{9 1 . 6 8}$ & $\mathbf{9 2 . 0 1}$ & $\mathbf{9 1 . 9 5}$ & $\mathbf{9 1 . 6 8}$ & $\mathbf{9 2 . 0 1}$ \\
D2 & 90.14 & 92.14 & 85.61 & 84.61 & $\mathbf{9 2 . 6 1}$ & $\mathbf{9 2 . 6 9}$ & $\mathbf{9 3 . 2 8}$ & $\mathbf{9 2 . 6 1}$ & $\mathbf{9 2 . 6 9}$ & $\mathbf{9 3 . 2 8}$ \\
D3 & 84.45 & 88.71 & 80.71 & 83.71 & 91.03 & 92.11 & 93.82 & $\mathbf{9 1 . 1 1}$ & $\mathbf{9 2 . 1 4}$ & $\mathbf{9 3 . 8 3}$ \\
D4 & 85.65 & 86.71 & 80.62 & 80.45 & 86.99 & $\mathbf{8 9 . 1 8}$ & 92.78 & $\mathbf{8 7 . 1 2}$ & 89.12 & $\mathbf{9 2 . 7 9}$ \\
D5 & 65.17 & 67.21 & 66.52 & 67.56 & 70.12 & 71.61 & $\mathbf{7 5 . 8 2}$ & $\mathbf{7 0 . 2 2}$ & $\mathbf{7 1 . 6 3}$ & 75.81 \\
D6 & 63.19 & 47.27 & 35.15 & 37.97 & 68.21 & 70.72 & 74.05 & $\mathbf{6 8 . 3 1}$ & $\mathbf{7 0 . 7 3}$ & $\mathbf{7 4 . 0 7}$ \\
D7 & 50.14 & 63.67 & 45.13 & 50.48 & $\mathbf{6 3 . 8 7}$ & 69.63 & $\mathbf{7 4 . 4 2}$ & $\mathbf{6 3 . 8 7}$ & $\mathbf{6 9 . 6 5}$ & 74.41 \\
D8 & 64.85 & 67.71 & 56.85 & 58.01 & 75.12 & 76.74 & 78.13 & 75.15 & $\mathbf{7 6 . 7 5}$ & $\mathbf{7 8 . 1 4}$ \\
Citeseer & 50.59 & 56.41 & 45.14 & 52.76 & $\mathbf{6 1 . 1 8}$ & $\mathbf{6 1 . 7 3}$ & $\mathbf{6 8 . 2 9}$ & $\mathbf{6 1 . 1 8}$ & $\mathbf{6 1 . 7 3}$ & $\mathbf{6 8 . 2 9}$ \\
Cora & 56.05 & 58.61 & 51.45 & 52.33 & $\mathbf{6 0 . 5 4}$ & $\mathbf{6 2 . 1 7}$ & $\mathbf{6 3 . 5 4}$ & $\mathbf{6 0 . 5 4}$ & $\mathbf{6 2 . 1 7}$ & $\mathbf{6 3 . 5 4}$ \\
\hline
\end{tabular}

\subsection{Summary}

This chapter presented two new clustering ensemble method (MDC and MOMVEC) based on multi-objective evolutionary algorithms. The methods introduced four new innovations: weighted intra-cluster distance in objective functions, row-wise/column-wise crossover methods, split/merge mutation methods and a tuning method in the multi-objective evolutionary process for refining the clustering solution.

MDC was implemented using SPEA-II and outperformed other clustering ensemble methods. Even when restricted to a single view, MDC provided better results on the majority of the datasets and was never worse. The use of multiple views generated a diverse set of clusters and led to 
even better results.

MOMVEC was implemented using NSGA-II and produced very similar results to MDC with slight improvements. The results showed that using a multi-objective approach is much better than using a single objective approach for clustering ensembles.

MDC and MOMVEC results performed on view one and view two produced different clustering solutions. The CA, F1 and RI values which represent the quality of clustering were different for both views. Generally view one (terms) dominated view two (hypertext), therefore we only showed the results of view one. However, in some cases the view two dominated the results. This variation of results on two views suggested that we should consider multiple views for clustering the data.

Simply concatenating the two views into a single feature matrix is not always a good solution. As shown in table 7.1, 7.2 and 7.3 the CA, F1 and RI values computed on D6 dataset for view v1+v2 was worse than 1 in case of Avg-Ensemble and MOCK clustering methods. Our methods MMOEA, MDC and MOMVEC used multiple views separately, therefore they got better results.

Our methods (MDC and MOMVEC) are able to produce both overlapping and non-overlapping clusters and automatically determines the number of clusters for final clustering solution. The current methods work well on small to medium size datasets having no more than 1500 features. Experiments indicate they are slow on large datasets with more then 4000 features. One of the remedies for this issue is to use dimensionality reduction techniques to reduce the number of features. However, using dimensionality reduction techniques may lead to loss of information. 


\section{Chapter 8}

\section{Conclusions}

Traditional clustering methods for text clustering are mainly originated from general clustering methods and usually ignore the rich features of text in clustering. The overall goal of this thesis was to develop novel clustering methods for text data that exploit the richness of the text.

The thesis achieved the goal by developing five new clustering methods that show how to utilize the rich features of the text in clustering to address the limitations of existing clustering methods. The newly developed text clustering methods were compared with state-of-the-art clustering methods and the results showed that these methods outperformed recent clustering methods. The following sections provide details of the achieved objectives, the main conclusions, future work and closing remarks for this thesis.

\subsection{Objectives and the Contributions}

The following four objectives were achieved by developing new clustering methods.

- Develop a new clustering method to exploit user queries for Search Result Clustering(SRC). 
Chapter 4 discussed the importance of using rich features in clustering methods to produce meaningful cluster labels and good quality clusters. Generally SRC methods ignore the fact that the user query is an important aspect in clustering the search results. When the user query is ambiguous, i.e., can have multiple senses, each sense represents a different meaning of the user query.

The thesis presented a new description-centric clustering method Query Sense Clustering (QSC) for SRC which achieved this objective.

QSC exploits query senses to generate meaningful cluster labels and uses two different text representations (based on syntactic and semantic features) of documents to generate quality clusters. QSC includes three steps to form a clustering solution-initial cluster generation, cluster optimization and cluster ranking.

QSC was compared with nine recent SRC methods and the result showed that QSC outperformed all other SRC methods by a large margin. The cluster labels generated by QSC were also more meaningful than STC and Lingo.

- Develop a new subspace clustering method for text data that uses semantic information in its distance measure.

A new soft subspace clustering method, Dirichlet Weighted K-means (DWKM), was presented in this thesis which achieved this objective. DWKM is based on the Latent Dirichlet Allocation (LDA) model and provides a new semantic feature weighting along with a new semantically weighted distance measure. DWKM uses Gibbs sampling to generate probability based topic-term and topic-document matrices which are then used to derive a probability based weighted distance measure for the clustering method. The DWKM algorithm uses the weighted distance measure to formulate clustering as a minimization problem and then produces the clustering solution using an iterative approach. 
DWKM was compared with simple k-means, LDA as clustering method, and three recent soft subspace clustering methods. The results showed that DWKM outperformed other clustering methods.

- Use multiple views of text data to generate diverse candidate clustering solutions for clustering ensemble methods.

A new clustering ensemble method Multi-View Multi-Objective Evolutionary Algorithm (MMOEA) was presented in the thesis which achieved this objective. In the first step, clustering ensemble methods try to generate multiple candidate solutions, which then are used in step two for deriving the final clustering solution. MMOEA introduced the concept of using multiple views of the text data to improve the first step of clustering ensemble methods.

In the second step, traditional clustering ensemble methods generally selected one candidate clustering solution from the generated candidate clustering solutions. However, MMOEA used NSGA-II for the second step and developed a cluster-centric approach to select quality clusters from different clustering solutions instead of selecting a single clustering solution to derive a final clustering solution. MMOEA was compared with state-of-the-art multi-objective clustering ensemble methods based on evolutionary algorithms and the results showed that MMOEA had a significant improvement over the previous methods.

- Develop a new approach to combining different candidate clustering solutions to generate final clustering solution for clustering ensemble methods. Two new multi-objective clustering ensemble methods Multi-objective Document Clustering (MDC) and Multi-objective Multi-view Ensemble Clustering (MOMVEC) were presented in the thesis which achieved this objective. In the first step, MDC uses a multi-view method similar to MMOEA to generate candidate clustering solutions, and in the second step, MDC introduces better crossover methods, muta- 
tion operators, a tuning step, two objective functions, and uses the SPEA-II algorithm.

MDC was compared with MMOEA and other recent multi-objective clustering ensemble methods. The result showed that MDC out performed MMOEA and other existing methods and had a significant improvement.

MOMVEC is similar to MDC except that it uses NSGA-II instead of the SPEA-II evolutionary algorithm in step 2 of the method. MOMVEC was compared with MDC and MMOEA and the result showed that MOMVEC had a significant improvement over existing clustering methods including MMOEA. However, MOMVEC and MDC performed equally well and MOMVEC has slightly better results compared to MDC.

\subsection{Main Conclusions}

This section provides the major conclusions of the four main contribution chapters (4-7) corresponding to the four objectives.

- Exploiting user queries improves Search Result Clustering (SRC)

Since utilizing different senses of user queries was the key change in QSC, the fact that QSC significantly outperformed existing SRC methods demonstrates that using the user query in this way can lead to improved results. Therefore, SRC methods should use the different senses to form clusters reflecting the different meanings of the user query.

- Index based evaluation measures may not be suitable for gold standard evaluation with an unbalanced number of clusters

Chapter 4 provides experiments using gold standards of AMBIENT and MORESQUE in the cluster analysis section. The gold standard 
has a coarse grain clustering solution while QSC produced fine grain clustering solutions. Since index based evaluation such as ARI and JI heavily penalize small clusters, using such evaluation measures can be misleading. Therefore, these index based evaluation measures are not suitable for evaluation if there is a mismatch in the grain size of the gold standard and the clustering solution. In such cases, we observed that F1-measure is more suitable for evaluating the quality of the clustering solution.

- Utilizing rich features in weighting schemes and distance measures improves soft subspace clustering

The distance measure is a crucial part of many clustering methods. Existing soft subspace methods for text clustering use general weighted distance measures. DWKM shows examples of how semantic information (rich features) can be used in deriving a probability based feature weighting scheme and a weighted distance measure for soft subspace clustering and demonstrates that using rich features in a weighting scheme and distance measure plays an important role in improving the quality of the clusters.

- Utilizing multiple views and a multi-objective cluster oriented method for combining solutions improves clustering ensemble methods

Chapter 6 discusses a clustering ensemble method MMOEA which uses multiple views to generate candidate clustering solutions. We demonstrated that using multiple views in step 1 of the clustering ensemble methods can lead to diverse candidate clustering solutions where the clusters from different views provide different insights about the data.

It was observed that some of the clusters in a clustering solution had a high compactness (documents were very similar) while other clusters had very low compactness. Because our cluster ensemble method uses multiple views to generate candidate clustering solu- 
tions, their candidate clustering solutions are also a mixture of high and low quality clusters. MMOEA shows that selecting high-quality clusters from a set of candidate clustering solutions, instead of selecting one candidate clustering solution in step two of the clustering method allows a clustering method to take advantage of the different insights of different views.

In step two, the common approach in existing clustering ensemble methods is to formulate a clustering problem as a single objective problem to select a candidate clustering solution as final clustering solutions. The single objective function is in fact based on different criteria which tries to measures intra-cluster similarity and intercluster separability using a linear function. MMOEA shows that formulating clustering problem as a multi-objective optimization approach to derive a clustering solution can improve the clustering ensemble methods.

- Better evolutionary operators and objective functions improve multi-objective evolutionary clustering ensemble methods

Chapter 7 provided details of two more clustering ensemble methods (MDC and MOMVEC), which were the improved version of MMOEA. The new methods had a better crossover, mutation, tuning and objective criteria than MMOEA, and results showed that the new methods had a significant improvement over MMOEA. Chapter 7 also provided an analysis of the method without crossover, mutation and tuning steps. We observed that removing these steps (crossover, mutation and tuning) causes worse results. Moreover, the single objective methods GA-1 and GA-2, mentioned in the chapter, also performed worse than MDC and MOMVEC. Hence, we concluded that better evolutionary operators and multi objective functions improve the results of evolutionary clustering ensemble methods. 


\subsection{Limitations and Future Work}

In general, this thesis sets a new direction for text clustering methods by encouraging researchers to use rich features in developing new and better clustering methods. Limitations and future directions are listed below for each newly developed clustering methods.

- Query Sense Clustering (QSC)

The similarity measure and document representation are the key factors in a clustering. QSC uses a simple sum and a scaling factor constant with cosine similarity function. The similarity measure of QSC could be improved by using a sophisticated method which automatically learns the value of the scaling factor.

The greedy search in step 2 of the QSC could also be improved to avoid local optima, by using the query senses in addition to document similarity. QSC used a Wikipedia based topic detection technique, which is not as good as state-of-the-art topic detection techniques such as LDA [15]; using LDA to detect topics from search results by considering query senses may further improve QSC.

QSC derives the user query senses from Wikipedia. The user query senses could also be derived from Google WebIT and ukWac corpus. In future, we would like to use Wikipedia, Google WebIT and ukWac corpus in conjunction to enhance the quality of query senses.

- Dirichlet Weighted K-means (DWKM)

Currently, DWKM requires users to input the number of topics to initialize the LDA model. In future, we will remedy this by investigating non-parametric LDA models and will try to reduce the computational complexity of the overall method. Another direction for DWKM is to investigate the use of LDA to generate different candidate clustering solutions for clustering ensemble methods. 
- Multi-View Multi-Objective Evolutionary Algorithm (MMOEA)

MMOEA is limited to domains that allow overlapping clusters and whose data can be represented by multiple views.

MMOEA currently requires a collection of documents which are retrieved by performing a query on the data. In the case of the Reuters data set for which there are no queries, a small modification would be required to disable the user query sense and the method could use two views (topics and terms) instead of three views.

MMOEA contains three objective functions which are computationally expensive. MMOEA could be reduced to two objective with a penalty criteria on the cluster size to improve the performance of the algorithm.

The genetic crossover operator used by MMOEA is very simple and the evolutionary search could be improved by adding different types of crossover and mutation operators which add, delete, replace, split and merge clusters. Furthermore, a tuning step in evolutionary process could be added which can fine tune the poor clusters.

Some limitations of MMOEA are addressed by MDC and MOMVEC.

- Multi-Objective Document Clustering (MDC) and MOMVEC

MDC and MMOEA work well on small to medium size datasets having no more than 1500 features but experiments indicate they are slow on large datasets with more than 4000 features. One of the remedies for this issue would be to use dimensionality reduction techniques to reduce the number of features. The difficulty is that, using dimensionality reduction techniques may lead to loss of information.

Future directions for both MDC and MOMVEC are to improve the scalability on larger corpora by applying an effective dimensionality reduction technique and to automatically identify the multiple 
views of the data without using any predefined views or domain knowledge.

\subsubsection{Future Work on Finding Multiple Views}

The current literature does not clearly define the concept of multiple views of the data. Some researchers refer to multiple views as different sets of features while others regard them as new features transformed from previous features.

Since this thesis was more focused on using rich features, one limitation of this thesis is that it does not address how to detect multiple views.

Most of the existing algorithms only handle disjoint views and ignore overlapping and hidden views. Disjoint views are generally formed by dividing features into different subsets. These subsets of features may provide a subspace where a cluster can be found, but there is no guarantee that these approaches will provide different views.

A common assumption for handling multiple views of existing algorithms is to consider all views have homogeneous data. However, the data is generally heterogeneous, which means objects can be described by categorical data, graph data, time series data or combination of different types of features. Furthermore, data can also come from different sources which can lead to more complexities in detecting multiple views. More details about multiple views and related literature can be found in [143, 121, 100].

The text clustering methods presented in this thesis use predefined multiple views. The primary future work for this thesis is to investigate how we can automatically detect multiple views in different datasets.

\subsection{Closing Remarks}

Traditional clustering methods such as k-means and AHC are widely used for clustering text data. Clustering text data poses additional challenges 
for clustering methods, which are usually not addressed by the traditional clustering methods. Although current text clustering methods try to address the challenges of text data, they ignore utilizing the rich features of the text data to improve clustering methods.

This thesis highlights the limitations of the current text clustering methods and discusses five new text clustering methods.

These new text clustering methods demonstrated that the rich features are very useful in clustering to determine the similarity of documents and can play an important role in deriving high quality clusters.

The new text clustering methods not only use the rich features but also address some of the limitations of the existing text clustering methods. These five clustering methods were compared with some of the existing text clustering methods and the result showed that the new text clustering methods outperformed other existing clustering methods. Hence, we concluded that utilizing rich features in clustering can produce better results and we encourage the future researchers to exploit rich features for improving text clustering methods. 
Appendices 



\section{Glossary}

Bag of words The bag of words approach states that a document or segment of text data is a bag which contains words of the document.. 22

Class A class is an ideal clusters in a gold standard clustering solution made by domain experts (human judges).. 40

Gold standard The gold standard clustering solution (also called ideal clustering solution) is generally made by domain experts (human judges). A common confusion in evaluation of clustering solution is to consider that the gold standard can be used during the clustering process. It is to note that the clustering is unsupervised learning and there is no information about the gold standard (ideal clustering solution) during the clustering process. The gold standard can only be used for evaluation after the clustering process is finished and a new clustering solution is generated. Clusters in gold standard are also called classes.. 40

N-grams The n-grams generally referred to as a sequence of the words occurring together in a sentence. The terms uni-grams, bi-grams and tri-grams are used to specify 1,2 and 3 word phrase. Whereas, the term n-grams is more general term and the value of $n$ represents number of words in a phrase.. 24 
VSM Vector Space Model uses the bag of words approach to represents all objects as vectors in a feature space. In text clustering, a document is a vector and all the words (vocabulary of the dataset) are features.. 5 


\section{Bibliography}

[1] Aggarwal, C. C., Wolf, J. L., Yu, P. S., Procopiuc, C., And PARK, J. S. Fast algorithms for projected clustering. In ACM SIGMOD Record (1999), vol. 28, ACM, pp. 61-72.

[2] Aggarwal, C. C., AND Yu, P. S. Finding generalized projected clusters in high dimensional spaces, vol. 29. ACM, 2000.

[3] Aggarwal, C. C., And Zhai, C. Mining text data. Springer Science \& Business Media, 2012.

[4] Aggarwal, C. C., And Zhai, C. A survey of text classification algorithms. In Mining text data. Springer, 2012, pp. 163-222.

[5] Agrawal, R., Gehrke, J., Gunopulos, D., and Raghavan, P. Automatic subspace clustering of high dimensional data for data mining applications, vol. 27. ACM, 1998.

[6] Aкано, S. A kernel method for canonical correlation analysis. arXiv preprint cs/0609071 (2006).

[7] Allen, R. B., ObRY, P., AND LitTman, M. An interface for navigating clustered document sets returned by queries. In Proceedings of the conference on Organizational computing systems (1993), ACM, pp. 166171.

[8] Bandyopadhyay, S., Maulik, U., AND Mukhopadhyay, A. Multiobjective genetic clustering for pixel classification in remote 
sensing imagery. Geoscience and Remote Sensing, IEEE Transactions on 45,5 (2007), 1506-1511.

[9] BARThÉLEMY, J.-P., AND LECLERC, B. The median procedure for partitions. DIMACS series in discrete mathematics and theoretical computer science 19 (1995), 3-34.

[10] Bernardini, A., CARpineto, C., And D'Amico, M. Fullsubtopic retrieval with keyphrase-based search results clustering. In Web Intelligence and Intelligent Agent Technologies, 2009. WI-IAT'09. IEEE/WIC/ACM International Joint Conferences on (2009), vol. 1, IET, pp. 206-213.

[11] Berry, M. W., ANd Castellanos, M. Survey of text mining. Computing Reviews 45, 9 (2004), 548.

[12] Bhattacharya, I., AND Getoor, L. A latent dirichlet model for unsupervised entity resolution. In SDM (2006), vol. 5, SIAM, p. 59.

[13] BiCKEL, S., AND SCHEFFER, T. Multi-view clustering. In Proceedings of the IEEE international conference on data mining (2004), vol. 36.

[14] BIEMANN, C. Chinese whispers: an efficient graph clustering algorithm and its application to natural language processing problems. In Proceedings of the First Workshop on Graph Based Methods for Natural Language Processing (2006), Association for Computational Linguistics, pp. 73-80.

[15] BLEI, D., NG, A., AND JordAN, M. Latent dirichlet allocation. the Journal of machine Learning research 3 (2003), 993-1022.

[16] BleI, D. M., AND LAfFerTy, J. D. Topic models. Text mining: classification, clustering, and applications 10 (2009), 71. 
[17] Blum, A., AND Mitchell, T. Combining labeled and unlabeled data with co-training. In Proceedings of the eleventh annual conference on Computational learning theory (1998), ACM, pp. 92-100.

[18] Branson, S., AND GreEnBerG, A. Clustering web search results using suffix tree methods. Tech. rep., Stanford University, Tech. Rep. CS276A Final Project, 2002.

[19] Carpineto, C., Osiński, S., Romano, G., AND Weiss, D. A survey of web clustering engines. ACM Computing Surveys (CSUR) 41, 3 (2009), 17.

[20] Carpineto, C., And Romano, G. Exploiting the potential of concept lattices for information retrieval with credo. Journal of universal computer science 10, 8 (2004), 985-1013.

[21] Carpineto, C., and Romano, G. Ambient dataset, 2008.

[22] Carpineto, C., And Romano, G. Optimal meta search results clustering. In Proceedings of the 33rd international ACM SIGIR conference on Research and development in information retrieval (2010), ACM, pp. 170-177.

[23] Chan, E. Y., Ching, W. K., NG, M. K., And Huang, J. Z. An optimization algorithm for clustering using weighted dissimilarity measures. Pattern recognition 37, 5 (2004), 943-952.

[24] Chaudhuri, K., KaKade, S. M., Livescu, K., And SRidharan, K. Multi-view clustering via canonical correlation analysis. In Proceedings of the 26th annual international conference on machine learning (2009), ACM, pp. 129-136.

[25] Chen, X., XU, X., Huang, J. Z., AND Ye, Y. Tw- $(k)$-means: Automated two-level variable weighting clustering algorithm for multiview data. Knowledge and Data Engineering, IEEE Transactions on 25, 4 (2013), 932-944. 
[26] Chen, X., YE, Y., XU, X., AND HuAnG, J. Z. A feature group weighting method for subspace clustering of high-dimensional data. Pattern Recognition 45, 1 (2012), 434-446.

[27] Cheng, C.-H., Fu, A. W., AND ZhANG, Y. Entropy-based subspace clustering for mining numerical data. In Proceedings of the fifth ACM SIGKDD international conference on Knowledge discovery and data mining (1999), ACM, pp. 84-93.

[28] Coello, C. A. C., Lamont, G. B., And Van Veldhuisen, D. A. Evolutionary algorithms for solving multi-objective problems. Springer, 2007.

[29] Corne, D. W., Jerram, N. R., Knowles, J. D., OAtes, M. J., ET AL. Pesa-ii: Region-based selection in evolutionary multiobjective optimization. In Proceedings of the Genetic and Evolutionary Computation Conference (GECCO2001 (2001), Citeseer.

[30] Crabtree, D., GaO, X., And Andreae, P. Improving web clustering by cluster selection. In Web Intelligence, 2005. Proceedings. The 2005 IEEE/WIC/ACM International Conference on (2005), IEEE, pp. 172-178.

[31] Crabtree, D., GaO, X., And Andreae, P. Query directed clustering. Knowledge and Information Systems (2012), 1-37.

[32] Croft, W. B., Turtle, H. R., And Lewis, D. D. The use of phrases and structured queries in information retrieval. In Proceedings of the 14th annual international ACM SIGIR conference on Research and development in information retrieval (1991), ACM, pp. 32-45.

[33] De Sa, V. R., Gallagher, P. W., Lewis, J. M., And MalaVe, V. L. Multi-view kernel construction. Machine learning 79, 1-2 (2010), 4771. 
[34] De Soete, G. Optimal variable weighting for ultrametric and additive tree clustering. Quality and Quantity 20, 2-3 (1986), 169-180.

[35] De Soete, G. Ovwtre: A program for optimal variable weighting for ultrametric and additive tree fitting. Journal of Classification 5, 1 (1988), 101-104.

[36] Deb, K., Pratap, A., Agarwal, S., and Meyarivan, T. A fast and elitist multiobjective genetic algorithm: Nsga-ii. Evolutionary Computation, IEEE Transactions on 6, 2 (2002), 182-197.

[37] DeSarbo, W. S., Carroll, J. D., Clark, L. A., and Green, P. E. Synthesized clustering: A method for amalgamating alternative clustering bases with differential weighting of variables. Psychometrika 49, 1 (1984), 57-78.

[38] Di Marco, A., And Navigli, R. Clustering web search results with maximum spanning trees. In $A I^{*} I A$ 2011: Artificial Intelligence Around Man and Beyond. Springer, 2011, pp. 201-212.

[39] Di Marco, A., AND NAVIGLI, R. Clustering and diversifying web search results with graph-based word sense induction. Computational Linguistics, Just Accepted (2013), 1-76.

[40] Domeniconi, C., Papadopoulos, D., Gunopulos, D., AND MA, S. Subspace clustering of high dimensional data. In SDM (2004), vol. 73, SIAM, p. 93.

[41] Dorow, B., Widdows, D., Ling, K., EckmanN, J.-P., Sergi, D., AND MOSES, E. Using curvature and markov clustering in graphs for lexical acquisition and word sense discrimination. arXiv preprint cond-mat/0403693 (2004).

[42] DutTA, D., DutTA, P., AND SIL, J. Simultaneous feature selection and clustering with mixed features by multi objective genetic algo- 
rithm. International Journal of Hybrid Intelligent Systems 11, 1 (2014), $41-54$.

[43] EIBEN, A. E., AND SMITH, J. E. Introduction to evolutionary computing. springer, 2003.

[44] Feldman, R., AND SANGER, J. The text mining handbook: advanced approaches in analyzing unstructured data. Cambridge University Press, 2007.

[45] FerraginA, P., AND GULLI, A. A personalized search engine based on web-snippet hierarchical clustering. In Special interest tracks and posters of the 14th international conference on World Wide Web (2005), ACM, pp. 801-810.

[46] Fortin, F.-A., AND PARIZEAU, M. Revisiting the nsga-ii crowdingdistance computation. In Proceedings of the 15th annual conference on Genetic and evolutionary computation (2013), ACM, pp. 623-630.

[47] FRED, A. L., AND JAIN, A. K. Data clustering using evidence accumulation. In Pattern Recognition, 2002. Proceedings. 16th International Conference on (2002), vol. 4, IEEE, pp. 276-280.

[48] Friedman, J. H., AND Meulman, J. J. Clustering objects on subsets of attributes (with discussion). Journal of the Royal Statistical Society: Series B (Statistical Methodology) 66, 4 (2004), 815-849.

[49] FRIEDMAN, M. The use of ranks to avoid the assumption of normality implicit in the analysis of variance. Journal of the American Statistical Association 32, 200 (1937), 675-701.

[50] Frigui, H., AND NASRaOUi, O. Simultaneous clustering and dynamic keyword weighting for text documents. In Survey of text mining. Springer, 2004, pp. 45-72. 
[51] FRIGUi, H., AND NASRAOUI, O. Unsupervised learning of prototypes and attribute weights. Pattern recognition 37, 3 (2004), 567-581.

[52] GAN, G., MA, C., AND WU, J. Data clustering: theory, algorithms, and applications, vol. 20. Siam, 2007.

[53] Ghosh, J., Strehl, A., And Merugu, S. A consensus framework for integrating distributed clusterings under limited knowledge sharing. In Proc. NSF Workshop on Next Generation Data Mining (2002), pp. 99-108.

[54] Giannotti, F., NAnni, M., Pedreschi, D., And SAmaritani, F. Webcat: Automatic categorization of web search results. SEBD03 (2003), 507-518.

[55] GODER, A., AND FILKOV, V. Consensus clustering algorithms: Comparison and refinement. In ALENEX (2008), vol. 8, pp. 109-117.

[56] Goil, S., Nagesh, H., And ChOudhary, A. Mafia: Efficient and scalable subspace clustering for very large data sets. In Proceedings of the 5th ACM SIGKDD International Conference on Knowledge Discovery and Data Mining (1999), pp. 443-452.

[57] GomAA, W. H., AND FAHMY, A. A. A survey of text similarity approaches. International Journal of Computer Applications 68, 13 (2013), 13-18.

[58] Guha, S., Rastogi, R., AND SHIM, K. Cure: an efficient clustering algorithm for large databases. In ACM SIGMOD Record (1998), vol. 27, ACM, pp. 73-84.

[59] Gullo, F., Domeniconi, C., And Tagarelli, A. Projective clustering ensembles. Data Mining and Knowledge Discovery 26, 3 (2013), 452-511. 
[60] Hammouda, K. M., AND Kamel, M. S. Efficient phrase-based document indexing for web document clustering. Knowledge and Data Engineering, IEEE Transactions on 16, 10 (2004), 1279-1296.

[61] Han, J., Kamber, M., AND PeI, J. Data mining: concepts and techniques. Morgan kaufmann, 2006.

[62] HANDL, J., AND KNOWLES, J. An evolutionary approach to multiobjective clustering. Evolutionary Computation, IEEE Transactions on 11, 1 (2007), 56-76.

[63] HANDL, J., AND KNOWLES, J. Evidence accumulation in multiobjective data clustering. In Evolutionary Multi-Criterion Optimization (2013), Springer, pp. 543-557.

[64] Hatzivassiloglou, V., And Wiebe, J. M. Effects of adjective orientation and gradability on sentence subjectivity. In Proceedings of the 18th conference on Computational linguistics-Volume 1 (2000), Association for Computational Linguistics, pp. 299-305.

[65] Hearst, M., and Pedersen, J. Reexamining the cluster hypothesis: scatter/gather on retrieval results. In Proceedings of the 19th annual international ACM SIGIR conference on Research and development in information retrieval (1996), ACM, pp. 76-84.

[66] HOU, J., AND NAYAK, R. The heterogeneous cluster ensemble method using hubness for clustering text documents. In International Conference on Web Information Systems Engineering (2013), Springer, pp. 102-110.

[67] HOU, J., AND NAYAK, R. Robust clustering of multi-type relational data via a heterogeneous manifold ensemble. In 2015 IEEE 31st International Conference on Data Engineering (2015), IEEE, pp. 615-626. 
[68] Hruschka, E. R., Campello, R. J. G. B., Freitas, A. A., And De Carvalho, A. P. L. F. A survey of evolutionary algorithms for clustering. Systems, Man, and Cybernetics, Part C: Applications and Reviews, IEEE Transactions on 39, 2 (2009), 133-155.

[69] HUANG, A. Similarity measures for text document clustering. In Proceedings of the sixth new zealand computer science research student conference (NZCSRSC2008), Christchurch, New Zealand (2008), pp. 4956.

[70] Hubert, L., AND ARABIE, P. Comparing partitions. Journal of classification 2, 1 (1985), 193-218.

[71] IAM-ON, N., BOONGOen, T., AND GarretT, S. Refining pairwise similarity matrix for cluster ensemble problem with cluster relations. In Discovery Science (2008), Springer, pp. 222-233.

[72] IAM-ON, N., AND GARRETT, S. Linkclue: A matlab package for link-based cluster ensembles. Journal of Statistical Software 36, i09 (2010).

[73] Jabeen, S., GaO, X., AND Andreae, P. Harnessing wikipedia semantics for computing contextual relatedness. In PRICAI 2012: Trends in Artificial Intelligence. Springer, 2012, pp. 861-865.

[74] Jain, A., AND Dubes, R. Algorithms for clustering data. PrenticeHall, Inc., 1988.

[75] Jain, A. K. Data clustering: 50 years beyond k-means. Pattern Recognition Letters 31, 8 (2010), 651-666.

[76] Jain, A. K., Murty, M. N., AND FlynN, P. J. Data clustering: a review. ACM computing surveys (CSUR) 31, 3 (1999), 264-323.

[77] JiNG, L., NG, M. K., AND HuANG, J. Z. An entropy weighting kmeans algorithm for subspace clustering of high-dimensional sparse 
data. Knowledge and Data Engineering, IEEE Transactions on 19, 8 (2007), 1026-1041.

[78] JinG, L., NG, M. K., XU, J., AND HuAnG, J. Z. Subspace clustering of text documents with feature weighting k-means algorithm. In Advances in Knowledge Discovery and Data Mining. Springer, 2005, pp. 802-812.

[79] Kaufman, L., AND RousseeuW, P. J. Finding groups in data: an introduction to cluster analysis, vol. 344. Wiley-Interscience, 2009.

[80] KÖPPEN, M., AND YOSHIDA, K. Substitute distance assignments in nsga-ii for handling many-objective optimization problems. In Evolutionary Multi-Criterion Optimization (2007), Springer, pp. 727741.

[81] Korkmaz, E. E., Du, J., AlhajJ, R., And BArker, K. Combining advantages of new chromosome representation scheme and multiobjective genetic algorithms for better clustering. Intelligent Data Analysis 10, 2 (2006), 163-182.

[82] KOWAlsKi, M., SzafransKi, M., And Ralaivola, L. Multiple indefinite kernel learning with mixed norm regularization. In Proceedings of the 26th Annual International Conference on Machine Learning (2009), ACM, pp. 545-552.

[83] KRIEGEL, H.-P., KRÖGER, P., AND ZimeK, A. Clustering highdimensional data: A survey on subspace clustering, pattern-based clustering, and correlation clustering. ACM Transactions on Knowledge Discovery from Data (TKDD) 3, 1 (2009), 1.

[84] KumAR, A., AND DAumÉ, H. A co-training approach for multiview spectral clustering. In Proceedings of the 28th International Conference on Machine Learning (ICML-11) (2011), pp. 393-400. 
[85] Kumar, A., Rai, P., AND Daume, H. Co-regularized multi-view spectral clustering. In Advances in Neural Information Processing Systems (2011), pp. 1413-1421.

[86] Kummamuru, K., Lotlikar, R., Roy, S., Singal, K., AND KRISHNAPURAM, R. A hierarchical monothetic document clustering algorithm for summarization and browsing search results. In Proceedings of the 13th international conference on World Wide Web (2004), ACM, pp. 658-665.

[87] Kuncheva, L., Hadjitodorov, S., and Todorova, L. Experimental comparison of cluster ensemble methods. In Information $\mathrm{Fu}$ sion, 2006 9th International Conference on (2006), IEEE, pp. 1-7.

[88] Kuncheva, L. I., AND BeZDeK, J. C. Selection of cluster prototypes from data by a genetic algorithm. In Proc. 5th European Congress on Intelligent Techniques and Soft Computing, Aachen, Alemanha (1997), pp. 1683-1688.

[89] LaW, M. H., TOPChY, A. P., AND JaIN, A. K. Multiobjective data clustering. In Computer Vision and Pattern Recognition, 2004. CVPR 2004. Proceedings of the 2004 IEEE Computer Society Conference on (2004), vol. 2, IEEE, pp. II-424.

[90] MaArek, Y. S., Fagin, R., Ben-Shaul, I. Z., AND Pelleg, D. Ephemeral document clustering for web applications.

[91] MacQueEn, J. B. Some methods for classification and analysis of multivariate observations. In Proc. of the fifth Berkeley Symposium on Mathematical Statistics and Probability (1967), L. M. L. Cam and J. Neyman, Eds., vol. 1, University of California Press, pp. 281-297.

[92] MakarenKov, V., AND Legendre, P. Optimal variable weighting for ultrametric and additive trees and k-means partitioning: Methods and software. Journal of Classification 18, 2 (2001), 245-271. 
[93] MASŁOWSKA, I. Phrase-based hierarchical clustering of web search results. In Advances in Information Retrieval. Springer, 2003, pp. 555562.

[94] MeILĂ, M. Comparing clusteringsan information based distance. Journal of Multivariate Analysis 98, 5 (2007), 873-895.

[95] Meiyappan, Y., IYengar, N. C. S. N., Kannan, A., Suyoto, Y. D., Suselo, T., Prasetyaningrum, T., Tlili, R., Slimani, Y., Dufreche, S., ZAPPI, M., ET AL. Srcluster: Web clustering engine based on wikipedia. International Journal of Advanced Science and Technology 39, 1 (2012), 1-18.

[96] Milne, D., And WitTen, I. H. An open-source toolkit for mining wikipedia. Artificial Intelligence (2012).

[97] Miner, G. Practical text mining and statistical analysis for nonstructured text data applications. Academic Press, 2012.

[98] Modha, D. S., AND SPANGLER, W. S. Feature weighting in kmeans clustering. Machine learning 52, 3 (2003), 217-237.

[99] Mukhopadhyay, A., MaUlik, U., AND BAndyOpadhyay, S. Multiobjective genetic algorithm-based fuzzy clustering of categorical attributes. Evolutionary Computation, IEEE Transactions on 13, 5 (2009), 991-1005.

[100] MÜller, E., GÜnNemanN, S., FÄrber, I., AND SeIdL, T. Discovering multiple clustering solutions: Grouping objects in different views of the data. In Data Engineering (ICDE), 2012 IEEE 28th International Conference on (2012), IEEE, pp. 1207-1210.

[101] NAvigli, R., AND CRisAfulli, G. Inducing word senses to improve web search result clustering. In Proceedings of the 2010 conference on empirical methods in natural language processing (2010), Association for Computational Linguistics, pp. 116-126. 
[102] NAYAK, R. Fast and effective clustering of $x m l$ data using structural information. Knowledge and Information Systems 14, 2 (2008), 197-215.

[103] NG, R. T., AND HAN, J. E cient and e ective clustering methods for spatial data mining. In Proc. of (1994), pp. 144-155.

[104] NGO, C. L., AND NGUYEN, H. S. A tolerance rough set approach to clustering web search results. In Knowledge Discovery in Databases: PKDD 2004. Springer, 2004, pp. 515-517.

[105] NGuyen, N., And Caruana, R. Consensus clusterings. In Data Mining, 2007. ICDM 2007. Seventh IEEE International Conference on (2007), IEEE, pp. 607-612.

[106] Nigam, K., AND GHANI, R. Analyzing the effectiveness and applicability of co-training. In Proceedings of the ninth international conference on Information and knowledge management (2000), ACM, pp. 8693.

[107] OSIRISKI, S., STEFANOWSKI, J., AND WeISS, D. Lingo: Search results clustering algorithm based on singular value decomposition. In Intelligent information processing and web mining: proceedings of the International IIS: IIPWM04 Conference held in Zakopane, Poland (2004), p. 359 .

[108] PANG-Ning, T., Steinbach, M., And KumaR, V. Introduction to data mining. WP Co (2006).

[109] PARK, J., GaO, X., And Andreae, P. Query directed web page clustering using suffix tree and wikipedia links. In Advanced Data Mining and Applications. Springer, 2012, pp. 91-99.

[110] Peng, G., FAnG, Y.-W., PenG, W.-S., ChaI, D., And Xu, Y. Multiobjective particle optimization algorithm based on sharing-learning 
and dynamic crowding distance. Optik-International Journal for Light and Electron Optics 127, 12 (2016), 5013-5020.

[111] Pirolli, P., Schank, P., Hearst, M., And Diehl, C. Scatter/gather browsing communicates the topic structure of a very large text collection. In Proceedings of the SIGCHI conference on $\mathrm{Hu}$ man factors in computing systems (1996), ACM, pp. 213-220.

[112] RipOn, K. S. N., TsAnG, C.-H., KwONG, S., AND IP, M.-K. Multiobjective evolutionary clustering using variable-length real jumping genes genetic algorithm. In Pattern Recognition, 2006. ICPR 2006. 18th International Conference on (2006), vol. 1, IEEE, pp. 1200-1203.

[113] Rosenberg, A., And Hirschberg, J. V-measure: A conditional entropy-based external cluster evaluation measure. In Proceedings of the 2007 Joint Conference on Empirical Methods in Natural Language Processing and Computational Natural Language Learning (EMNLPCoNLL) (2007), vol. 410, p. 420.

[114] SAlton, G., AND MCGILL, M. J. Introduction to modern information retrieval.

[115] SAlton, G., Wong, A., AND YANG, C.-S. A vector space model for automatic indexing. Communications of the ACM 18, 11 (1975), 613-620.

[116] StefanOWSKI, J., AND WeISS, D. Carrot2 and language properties in web search results clustering. In Advances in Web Intelligence. Springer, 2003, pp. 240-249.

[117] SteIN, B., AND ZU EISSEN, S. M. Topic identification: Framework and application. In Proc. International Conference on Knowledge Management (2004), vol. 400, pp. 522-531.

[118] Steyvers, M., AND GRIFFITHS, T. Probabilistic topic models. Handbook of latent semantic analysis 427, 7 (2007), 424-440. 
[119] Strehl, A., AND Ghosh, J. Cluster ensembles-a knowledge reuse framework for combining multiple partitions. The Journal of Machine Learning Research 3 (2003), 583-617.

[120] SUN, S. Multi-view laplacian support vector machines. Advanced Data Mining and Applications (2011), 209-222.

[121] Sun, S. A survey of multi-view machine learning. Neural Computing and Applications 23, 7-8 (2013), 2031-2038.

[122] SUn, S., AND SHAWE-TAYLOR, J. Sparse semi-supervised learning using conjugate functions. The Journal of Machine Learning Research 11 (2010), 2423-2455.

[123] SZAFranski, M., GRAndValet, Y., and Rakotomamonjy, A. Composite kernel learning. Machine learning 79, 1-2 (2010), 73-103.

[124] ToPchY, A., JAIN, A. K., AND PUnCH, W. A mixture model of clustering ensembles. In Proc. SIAM Intl. Conf. on Data Mining (2004), Citeseer.

[125] Topchy, A., JaIN, A. K., AND PUnCH, W. Clustering ensembles: Models of consensus and weak partitions. Pattern Analysis and Machine Intelligence, IEEE Transactions on 27, 12 (2005), 1866-1881.

[126] TRan, T., NAyAK, R., AND BRUZA, P. Combining structure and content similarities for $\mathrm{xml}$ document clustering. In Proceedings of the 7th Australasian Data Mining Conference-Volume 87 (2008), Australian Computer Society, Inc., pp. 219-225.

[127] TzORTZIS, G. F., AND LIKAS, A. C. The global kernel-means algorithm for clustering in feature space. Neural Networks, IEEE Transactions on 20, 7 (2009), 1181-1194. 
[128] Vega-Pons, S., CORReA-Morris, J., AND RuIZ-ShulCloper, J. Weighted partition consensus via kernels. Pattern Recognition 43, 8 (2010), 2712-2724.

[129] Vega-PONS, S., AND RuIZ-ShulClOPER, J. A survey of clustering ensemble algorithms. International Journal of Pattern Recognition and Artificial Intelligence 25, 03 (2011), 337-372.

[130] VÉRONIS, J. Hyperlex: lexical cartography for information retrieval. Computer Speech E Language 18, 3 (2004), 223-252.

[131] WAHID, A., GAO, X., AND AndreaE, P. Exploiting user queries for search result clustering. In Web Information Systems EngineeringWISE 2013. Springer, 2013, pp. 111-120.

[132] Wahid, A., GaO, X., AND Andreae, P. Multi-view clustering of web documents using multi-objective genetic algorithm. In Evolutionary Computation (CEC), 2014 IEEE Congress on (2014), IEEE, pp. 2625-2632.

[133] WAHID, A., GAO, X., AND AndreaE, P. Multi-objective clustering ensemble for high-dimensional data based on strength pareto evolutionary algorithm (spea-ii). In Data Science and Advanced Analytics (DSAA), 2015. 36678 2015. IEEE International Conference on (2015), IEEE, pp. 1-9.

[134] WAHID, A., GAO, X., AND ANDREAE, P. Multi-objective multi-view clustering ensemble based on evolutionary approach. In Evolutionary Computation (CEC), 2015 IEEE Congress on (2015), IEEE, pp. 1696 $-1703$.

[135] WAHID, A., GAO, X., AND ANDreaE, P. A soft subspace clustering method for text data using a probability based feature weighting scheme. In Web Information Systems Engineering-WISE 2015. Springer, 2015, pp. 124-138. 
[136] Wang, H., Shan, H., AND BAnerJee, A. Bayesian cluster ensembles. Statistical Analysis and Data Mining 4, 1 (2011), 54-70.

[137] WANG, X., YANG, C., AND ZHOU, J. Clustering aggregation by probability accumulation. Pattern Recognition 42, 5 (2009), 668-675.

[138] WiLCOXON, F. Individual comparisons by ranking methods. Biometrics bulletin (1945), 80-83.

[139] WoO, K.-G., LeE, J.-H., KIM, M.-H., AND LeE, Y.-J. Findit: a fast and intelligent subspace clustering algorithm using dimension voting. Information and Software Technology 46, 4 (2004), 255-271.

[140] WoraWitphinYo, P., GAO, X., AND JABEen, S. Improving suffix tree clustering with new ranking and similarity measures. Advanced Data Mining and Applications (2011), 55-68.

[141] WU, Z., AND PALMER, M. Verbs semantics and lexical selection. In Proceedings of the 32nd annual meeting on Association for Computational Linguistics (1994), Association for Computational Linguistics, pp. 133-138.

[142] XIE, X. L., AND BENI, G. A validity measure for fuzzy clustering. Pattern Analysis and Machine Intelligence, IEEE Transactions on 13, 8 (1991), 841-847.

[143] $\mathrm{XU}, \mathrm{C} ., \mathrm{TAO}, \mathrm{D} ., \mathrm{AND} \mathrm{XU}_{\mathrm{U}} \mathrm{C}$. A survey on multi-view learning. arXiv preprint arXiv:1304.5634 (2013).

[144] YANG, J., WANG, W., WANG, H., AND YU, P. $\delta$-clusters: Capturing subspace correlation in a large data set. In Data Engineering, 2002. Proceedings. 18th International Conference on (2002), IEEE, pp. 517-528.

[145] YoON, H.-S., AHN, S.-Y., LeE, S.-H., CHO, S.-B., AND KIM, J. H. Heterogeneous clustering ensemble method for combining different 
cluster results. In Data Mining for Biomedical Applications. Springer, 2006, pp. 82-92.

[146] ZAMIR, O., AND ETZIONI, O. Web document clustering: a feasibility demonstration. In Proceedings of the 21st annual international ACM SIGIR conference on Research and development in information retrieval (New York, NY, USA, 1998), SIGIR '98, ACM, pp. 46-54.

[147] ZamiR, O., EtziOni, O., MAdAni, O., AND KARP, R. Fast and intuitive clustering of web documents. In Proceedings of the $3 \mathrm{rd}$ International Conference on Knowledge Discovery and Data Mining (1997), MIT Press, pp. 287-290.

[148] ZENG, H.-J., HE, Q.-C., CHEN, Z., MA, W.-Y., AND MA, J. Learning to cluster web search results. In Proceedings of the 27 th annual international ACM SIGIR conference on Research and development in information retrieval (2004), ACM, pp. 210-217.

[149] Zhai, C. X., COHEN, W. W., AND LAfFerTy, J. Beyond independent relevance: methods and evaluation metrics for subtopic retrieval. In Proceedings of the 26th annual international ACM SIGIR conference on Research and development in informaion retrieval (2003), ACM, pp. 10-17.

[150] ZHANG, T., RAMAKRISHNAN, R., AND LIVNY, M. Birch: an efficient data clustering method for very large databases. In ACM SIGMOD Record (1996), vol. 25, ACM, pp. 103-114.

[151] ZHANG, X., Hu, X., AND ZHOU, X. A comparative evaluation of different link types on enhancing document clustering. In Proceedings of the 31st annual international ACM SIGIR conference on Research and development in information retrieval (2008), ACM, pp. 555-562.

[152] ZHAO, Y., AND KARYPIS, G. Criterion functions for document clustering: Experiments and analysis. Machine Learning (2001). 
[153] ZhaO, Y., AND KARYPIS, G. Comparison of agglomerative and partitional document clustering algorithms. Tech. rep., DTIC Document, 2002.

[154] ZHONG, S., AND GHOSH, J. A comparative study of generative models for document clustering. In Proceedings of the workshop on Clustering High Dimensional Data and Its Applications in SIAM Data Mining Conference (2003).

[155] Zitzler, E., Laumanns, M., Thiele, L., ZitZler, E., ZitZler, E., Thiele, L., AND Thiele, L. Spea2: Improving the strength pareto evolutionary algorithm, 2001. 